\title{
Antifouling Strategies for Selective In Vitro and In Vivo Sensing
}

\author{
${ }_{2}$ Cheng Jiang, ${ }^{\text {If }}$ Guixiang Wang, ${ }^{\text {II }}$ Robert Hein, ${ }^{\text {II }}$ Nianzu Liu, Xiliang Luo,* and Jason J. Davis*
}

Cite This: https://dx.doi.org/10.1021/acs.chemrev.9b00739

Read Online

3 ABSTRACT: The ability to fabricate sensory systems capable of highly selective operation 4 in complex fluid will undoubtedly underpin key future developments in healthcare. 5 However, the abundance of (bio)molecules in these samples can significantly impede 6 performance at the transducing interface where nonspecific adsorption (fouling) can both 7 block specific signal (reducing sensitivity) and greatly reduce assay specificity. Herein, we 8 aim to provide a comprehensive review discussing concepts and recent advances in the 9 construction of antifouling sensors that are, through the use of chemical, physical, or 10 biological engineering, capable of operating in complex sample matrix (e.g., serum). We 11 specifically highlight a range of molecular approaches to the construction of solid sensory 12 interfaces (planar and nanoparticulate) and their characterization and performance in 13 diverse in vitro and in vivo analyte (e.g., proteins, nucleic acids, cells, neuronal transmitters)

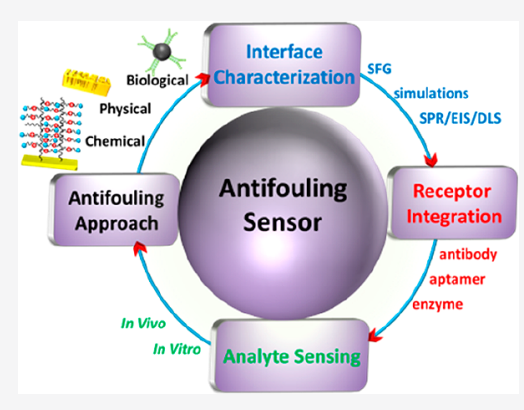
14 detection applications via derived selective optical or electrochemical strategies. We specifically highlight those sensors that are 15 capable of detection in complex media or those based on novel architectures/approaches. Finally, we provide perspectives on future 16 developments in this rapidly evolving field.

\section{CONTENTS}

19 1. Introduction

20 2. Chemical Antifouling Strategies and Their Sensing Applications

2.1. Selection and Immobilization of Antifouling Agents

2.1.1. Choice of Antifouling Agent

2.1.2. Immobilization Strategies

2.2. Characterization of Antifouling Surfaces

2.2.1. Probing Antifouling Mechanism

2.2.2. Evaluation of Antifouling Behavior

2.3. Sensors Based on Chemical Antifouling Interfaces

2.3.1. Incorporation of Receptive Elements

2.3.2. In Vitro Sensing Based on Antifouling Chemistries

2.3.3. In Vivo Sensing Based on Antifouling Chemistries

36 3. Sensors Based on Physical Antifouling Strategies

3.1. Porous Transducer Topographies

3.2. Filtration Methodologies

39 4. Sensors Based on Biological Antifouling Approaches

4.1. Affinity Depletion

4.2. Degradation by Enzyme Catalysis

43 5. Conclusions and Perspectives

5.1. Exploration of New Antifouling Materials and Approaches for Sensing

5.2. Simulation Assisted Sensory Design and Data Analytics
5.3. Highly Integrated PoC Sensing Devices

Author Information

Corresponding Authors

Authors

Author Contributions

Notes

Biographies

Acknowledgments

References

AB 48

AB 49

$A B 50$

AB 51

AB 52

AC 53

AC 54

AC 55

AC 56

\section{INTRODUCTION}

$\mathrm{H} \quad$ It is undoubtedly the case that an early diagnosis and treatment 57

$\mathrm{H}$ of disease can dramatically reduce mortality and human 58 suffering. A detection of markers shed from tumors, for 59 example, provides a potent opportunity to diagnose cancer 60 early and disease management. ${ }^{1-4}$ However, one major 61 challenge is that in complex biological fluids (such as serum, 62 saliva, urine, etc.) or in the in vivo circulating environment, the 63 concentration of disease markers (e.g., specific proteins) is 64 often very low in comparison to the large number of coexisting 65 background species (e.g., proteins, cells) that can adsorb onto 66 a sensing interface nonspecifically. This can cause serious 67

Received: November 13, 2019 
68 operational interference such as false positives, lowered signal69 to-noise, and impairing specific recognition/response. ${ }^{5-7}$ 70 Further side effects can arise for implantable sensors by, for 71 example, thrombus formation. ${ }^{8,9}$ There are a number of ways 72 of combatting this issue by preventing nonspecific binding 73 (fouling) while retaining specific binding. The primary one 74 that does not require sample pretreatment is to integrate 75 nonfouling chemistry into the assaying surfaces. ${ }^{10,11}$ The use of 76 such materials has received increasing attention in recent years, 77 where poly(ethylene glycol) (PEG) and its derivatives, ${ }^{12-14}$ 78 zwitterionic materials, ${ }^{15-17}$ peptides and peptoids, ${ }^{18-20}$ and 79 others candidates (e.g., hyaluronic acid (HA), polyoxazoline, 80 etc. $)^{21-24}$ have all been applied. Significantly, this has 81 facilitated a quantification of clinically relevant targets in 82 complex media via a number of transducer modalities and with 83 minimal sample pretreatment. In general terms, it is worth 84 noting that no existing antifouling strategy can completely 85 prevent fouling, and indeed this is not a requirement so long as 86 functionality is primarily retained over the relevant time frame 87 of application and any signal-generating "background noise" 88 that arises from nonspecific surface association falls well below 89 the required detection limits. Moreover, as in vivo biofouling 90 (i.e., protein corona) is inherent in the action of the immune 91 system, complete inhibition may lead to unintended 92 physiological responses and even damage to the host. ${ }^{25,26}$ It 93 should also be noted that different sensory formats suffer 94 differently from fouling. For example, sandwich assays are 95 much less sensitive to fouling so long as fouling does not 96 impede the primary (capture) antibody. In contrast, label-free 97 sensors, such as those based on surface-plasmon resonance 98 (SPR) or electrochemical impedance spectroscopy (EIS), 99 cannot distinguish between specifically bound target analytes 100 and other adsorbed interferents and are, then, particularly 101 sensitive to the deleterious impact of fouling. It is thus 102 imperative that, for any desired sensing application, the 103 potential impact of nonspecific adsorption is carefully 104 considered. In general, a large variety of relevant characteristics 105 should be considered and optimized for; these include 106 sensitivity, selectivity, reproducibility, dynamic range, lifetime, 107 baseline stability, ease-of-fabrication, and response time. In 108 different sensor formats, the impact of fouling on these 109 parameters can be considerably different such that it will often 110 be necessary to rank parameters that are more or less crucial to 111 the chosen application. For example, in an implantable sensor, 112 its lifetime and toxicology are of crucial importance, while, in 113 ultrasensitive single molecule sensors, the sensitivity (signal-to114 noise) at ultralow concentrations is more important (see Table $1151)$.

116 In this review, we focus on recent advances in the 117 construction of solid-phase sensors capable of operation in 118 complex biological fluid. We comprehensively discuss a range 119 of antifouling approaches with a particular focus on the most 120 commonly used zwitterion- and PEG-based highly hydrated 121 chemical surface modifiers. In addition to these chemical 122 approaches, physical (e.g., substrate topography engineering or 123 membrane filtration) and biological approaches (e.g., bio124 affinity depletion or enzyme catalyzed degradation) will be 125 discussed. Construction methods, including those based on 126 self-assembly, (electro)polymerization, and electrografting, will 127 be highlighted as well as the integration of specific (bio)128 recognition elements. The experimental and theoretical 129 methods that have been employed to study these architectures 130 and their associated antifouling performance are also
Table 1. Overview of Important Sensory Parameters in the Context Specific Sensor Antifouling Performance

\begin{tabular}{|c|c|}
\hline sensor type & principle challenges $^{a}$ \\
\hline $\begin{array}{l}\text { general sensors, PoC/disposable } \\
\text { sensors }\end{array}$ & $\begin{array}{l}\text { sensitivity } \\
\text { selectivity } \\
\text { reproducibility } \\
\text { response time } \\
\text { fabrication cost } \\
\text { degree of user intervention }\end{array}$ \\
\hline $\begin{array}{l}\text { ultrasensitive and single molecule } \\
\text { sensors }\end{array}$ & $\begin{array}{l}\text { high signal/noise at ultralow } \\
\text { concentration } \\
\text { data magnitude } \\
\text { scalability }\end{array}$ \\
\hline $\begin{array}{l}\text { durable (e.g., implantable, wearable) } \\
\text { sensors }\end{array}$ & $\begin{array}{l}\text { selectivity } \\
\text { operation duration } \\
\text { immunogenicity }\end{array}$ \\
\hline
\end{tabular}

${ }^{a}$ It is important to note we have highlighted the principle challenges that are (uniquely) associated with the specific sensor type. Many general assay characteristics, such as a required sensitivity and selectivity, will be common to all.

described. Lastly, we discuss a number of specific applications 131 of so derived in vitro and in vivo sensors capable of sensing 132 clinically relevant target analytes such as proteins, nucleic acids, 133 or circulating small molecules like dopamine or glucose.

\section{CHEMICAL ANTIFOULING STRATEGIES AND THEIR SENSING APPLICATIONS}

One of the most commonly employed strategies to endow 136 sensory interfaces with antifouling properties is the use of 137 chemical modifiers that render the interface strongly hydrated. 138 This hydration has been associated with a resistance to fouling 139 (as discussed in more detail in Section 2.2) and is generated by 140 decorating the sensory interfaces with highly polar, hydrated 141 chemical groups/materials. In this section, we will discuss the 142 most commonly used chemical agents, their incorporation into 143 sensors, and the evaluation of their antifouling properties. 144

\subsection{Selection and Immobilization of Antifouling Agents}

2.1.1. Choice of Antifouling Agent. As shown in Figure $145 \mathrm{fl}$ 1 , a wide range of molecular systems, such as polyethylene- $146 \mathrm{fl}$ glycol (PEG, >10 EG units) and its derivatives, zwitterionic 147 species (phosphorylcholine (PC), sulfobetaine (SB), and 148 carboxybetaine $(\mathrm{CB})$ ), peptides with alternating or random 149 mixed-charge (based on glutamic or aspartic acid (E/D) and 150 lysine (K), e.g., EKEKEKEC), ${ }^{27}$ and other polymers like 151 polysaccharides, polyoxazolines, poly(hydroxy acrylates), 152 hyperbranched polyglycerol (HPG), or hybrid materials (e.g., 153 PEG-SB) have been shown to possess significant antifouling 154 properties that arise primarily from their strong hydration (see 155 Section 2.2). ${ }^{28-31}$ All of these systems have been employed for 156 a variety of purposes and possess specific features and 157 advantages/disadvantages in terms of derived specific sensing 158 (discussed in more detail in Section 2.3). We will first 159 introduce the means by which these chemistries can be 160 incorporated into interfaces and then how this can be 161 characterized.

2.1.2. Immobilization Strategies. As shown in Figure 2, $163 \mathrm{f} 2$ the immobilization of an antifouling coating onto a solid 164 substrate can be achieved through a range of methods. Most 165 commonly, self-assembly, ${ }^{32-34}$ electrografting, ${ }^{35,36}$ or polymer- 166 ization (e.g., electropolymerization, ${ }^{37,38}$ atom transfer radical 167 polymerization (ATRP), ${ }^{39-43}$ or reversible addition-fragmen- 168 


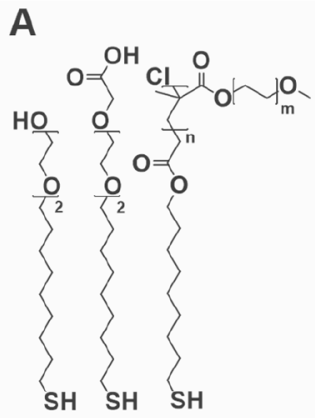<smiles>[R]N(C)c1ccccc1N=[R]</smiles>
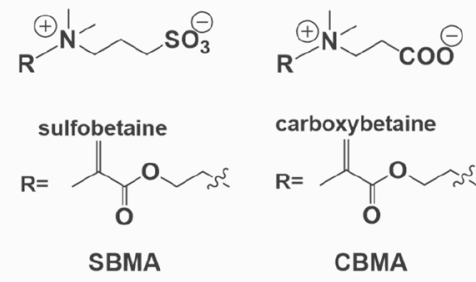

phosphorylcholine

$R=\widehat{H S}_{\text {SB-thiol }} \widehat{N}_{\mathbf{n}}^{\mathrm{r}_{\mathrm{i}}}$

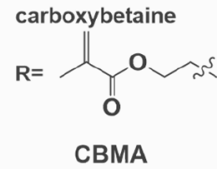

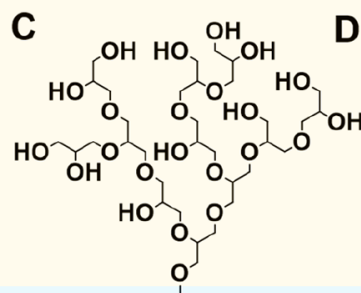

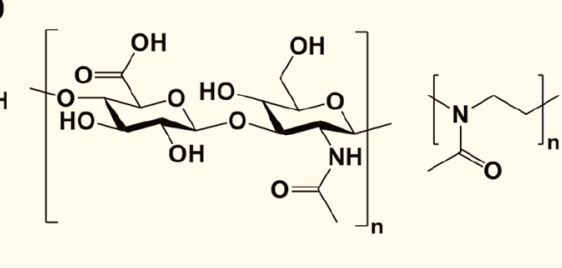

$R=H S \widehat{n n}$

CB-thiol

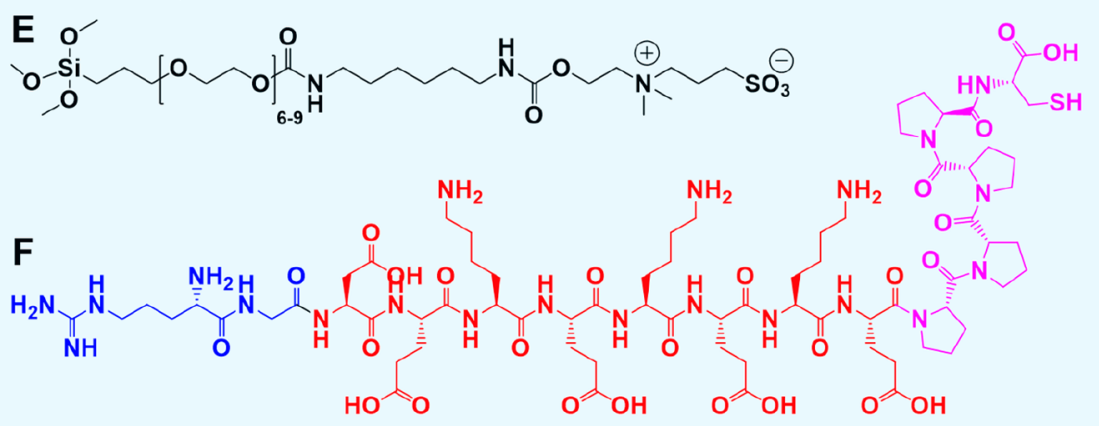

Figure 1. Chemical structures of (A) OEG/PEG based thiols, (B) zwitterionic molecular systems, (C) hyperbranched polyglycerol (HPG), (D) hyaluronic acid (HA), poly(2-methyl-2-oxazoline) and poly(2-hydroxyethyl methacrylate) (pHEMA) (from left to right), (E) PEG-SB silane, and (F) an all-in-one natural mixed peptide containing a cell recognition sequence (RGD, blue), antifouling unit (EKEKEKE, red), and an anchoring sequence (CPPPP, magenta).

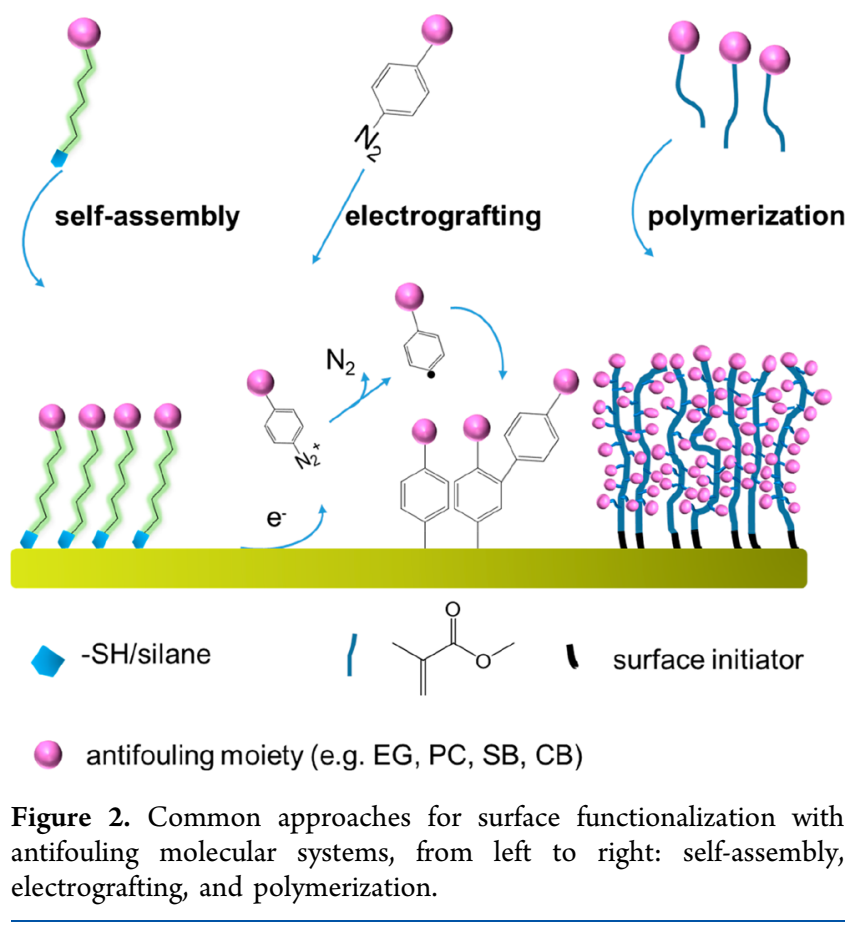

169 tation chain transfer (RAFT) $)^{44,45}$ ) methodologies are applied. 170 Importantly, an extensive variety of nonfouling architectures can be incorporated into sensors utilizing these approaches if 171 they are endowed with the appropriate chemical functionalities 172 (e.g., thiols, silanes, or (meth)acrylates). In prior considering 173 an immobilization protocol, both the specific underlying 174 substrate and the required film characteristics (hydration, 175 chemical stability, biological functionalization etc.) should be 176 taken into account. One of the most common methods to 177 immobilize antifouling components is self-assembly via 178 "thiolate" bonding at noble metal surfaces. ${ }^{33,34,46,47}$ This 179 well-established methodology offers easy access to well- 180 defined, monolayer architectures via simple exposure of clean 181 (commonly gold) substrates to solutions or vapors of thiol- 182 derived organics. Though deemed simple and undoubtedly 183 ubiquitous, it is, however, limited in terms of substrate scope. 184 In contrast, polymeric antifouling materials have been explored 185 extensively and can be applied to a broad range of interfaces 186 via graft-to and graft-from strategies whereby not only various 187 monomers (most commonly (meth)acrylates) can be used but 188 various film parameters, such as thickness and density can 189 often be controlled easily. ${ }^{12,48-50} 190$

Another commonly used strategy to functionalize con- 191 ductive interfaces (relevant to electronic/electrochemical or 192 plasmon resonance sensing) is electrografting. ${ }^{51}$ For example, 193 the reductive grafting of diazonium salts, which can be 194 presynthesized or generated in situ from the corresponding 195 aniline, is a convenient method to functionalize a variety of 196 
197 (electrode) materials such as carbon, gold, or platinum. 198 Specifically, the electrografting of arydiazonium salts proceeds 199 via the formation of an aryl radical, which spontaneously reacts 200 with the substrate to form a covalent bond. ${ }^{52}$ Depending on 201 the grafting conditions (time, potential range, concentration, 202 etc.), films of variable thickness can be obtained. This 203 methodology can be applied to form homogeneous (single 204 component) ${ }^{53}$ or heterogeneous films (multiple compo205 nents). ${ }^{54}$ Antifouling moieties, for example, ethylene glycol $206(\mathrm{EG})^{55}$ or zwitterionic groups (phosphoryl choline (PC), 207 sulfobetaine $(\mathrm{SB})$, carboxybetaine $(\mathrm{CB})),^{35,56,57}$ at the para208 position of the corresponding aniline precursors can be grafted 209 and can endow the resultant interface with the desired 210 antifouling properties.

\subsection{Characterization of Antifouling Surfaces}

211 In recent years, great progress has been made in elucidating the 212 processes that govern fouling interactions at interfaces and how 213 these can be modulated through (chemical) modification. The 214 widely accepted view is that entropic loss associated with 215 surface group mobility upon fouling (steric suppression) and 216 the intrinsic strong hydration of these films play key roles 217 wherein a tightly bound water layer, that is, hydration layer, 218 forms a physical and energetic barrier to (protein) adsorption 219 (Figure 3). ${ }^{15,58}$ Where neutral hydrophilic components are
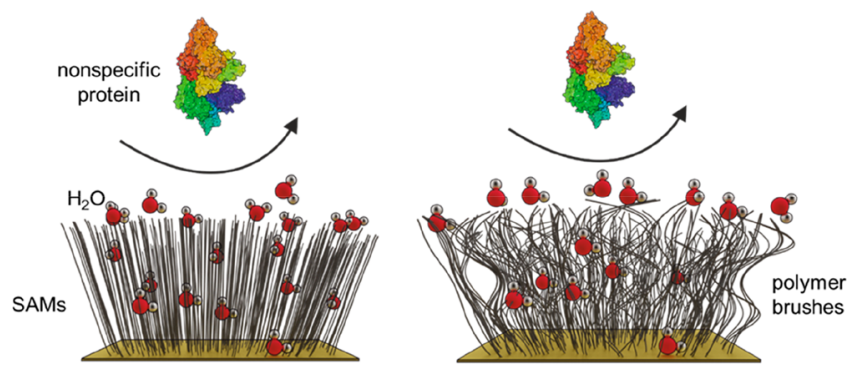

Figure 3. Antifouling molecular films present highly hydrated functionalities (e.g., EG, zwitterionic $\mathrm{PC} / \mathrm{SB} / \mathrm{CB}$ ), which are implicit in protein resistance.

220 used (such as PEG chains), this hydration is tethered via 221 hydrogen bonding. ${ }^{12,59}$ When charged (e.g., zwitterionic) 222 interfaces are used, the hydration layer has a large electrostatic 223 component. ${ }^{60-62}$ These are generalized observations/assump224 tions that populate an accepted picture without providing 225 mechanistic detail. Understanding the specific structure of 226 surface hydration and other interfacial processes/character227 istics is valuable in guiding the design of new and effective 228 nonfouling materials for robust sensing in complex environ229 ments. The following section will briefly discuss nonfouling 230 mechanisms and the correlation between interfacial structure 231 and nonspecific surface association as supported by simulations 232 and experimental studies. It should be noted that most of these 233 studies are carried out on chemical antifouling systems but that 234 the experimental techniques that will be discussed can often 235 also be utilized to evaluate the performance of other 236 (nonchemical) systems such as that provided by porous 237 electrodes (Section 3.1).

238 2.2.1. Probing Antifouling Mechanism. 2.2.1.1. Exper239 imental Analyses. A number of studies have been reported to 240 directly probe the hydration layer of a range of nonfouling 241 chemical entities. In solution nuclear magnetic resonance 242 (NMR) spectroscopy has been utilized to, for example, study the hydration of poly (sulfobetaine methacrylate) (pSBMA) or 243 PEG, however it is it is unclear to what extent these results are 244 transferable to a solid/liquid nonfouling interface where film 245 thickness, packing density, chain conformation and local chain 246 flexibility effects are very likely to be significant. ${ }^{63-65}$ A direct 247 insight into the hydration of interfaces can be provided by sum 248 frequency generation (SFG) vibrational spectroscopy. SFG 249 vibrational spectroscopy is surface-sensitive technique ${ }^{66}$ and, 250 unlike other methods, which cannot distinguish between 251 interfacial and bulk water, it facilitates a selective investigation 252 of the properties of the former. This is achieved by irradiation 253 of the surface with two laser beams which generate a mixed 254 output signal offering information complementary to that 255 offered by more standard IR and Raman spectroscopies 256 (Figure 4). ${ }^{67,68}$ This has for example been exploited by Leng et $257 \mathrm{f4}$

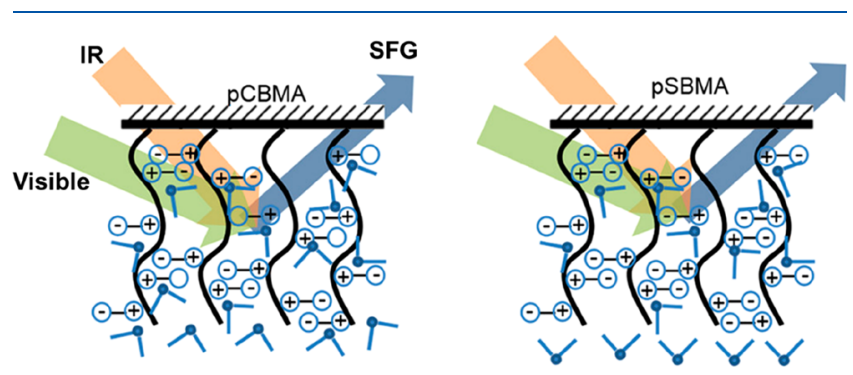

Figure 4. Schematic representation of the investigation of water molecules on PCBMA and PSBMA coated silica prism by SFG. Reproduced with permission from ref 76. Copyright 2018 American Chemical Society.

al., who used SFG for an in situ and real-time assessment of the 258 surface hydration of pSBMA and OEG methacrylate 259 (OEGMA) polymer brushes at $\mathrm{SiO}_{2}$ /liquid interfaces exposed 260 to protein solution. ${ }^{69,70}$ The SFG intensity is correlated with 261 the ordering of the dipole moments of the interfacial functional 262 groups selected and thus allows a direct interrogation of water 263 molecule ordering at the interface; a strong SFG signal for 264 water thus indicates both high levels of ordering and of 265 hydration. ${ }^{71-74}$ In Leng's work, it was observed that, although 266 strongly bonded water exists at both interfaces, the surface 267 hydration of PSBMA remained unaffected while the water 268 ordering at the pOEGMA surface was disturbed upon exposure 269 to protein ( $1 \mathrm{mg} \mathrm{mL} \mathrm{m}^{-1}$ of bovine serum albumin (BSA), 270 lyzosome or fibrinogen). ${ }^{70}$ Wang et al. have similarly applied 271 SFG to confirm that interfacial water molecule presence within 272 EG based self-assembled monolayer (SAM) films. ${ }^{75} 273$

In another study from Leng et al., water molecules were 274 shown to be strongly hydrogen-bonded to zwitterionic 275 interfaces (poly (carboxybetaine acrylamide) (pCBAA) and 276 pSBMA), much more than at pOEGMA (which contained a 277 detectable amount of weakly hydrogen-bonded water). ${ }^{69}$ Chen 278 and co-workers further elucidated the water structure on 279 nonfouling poly(carboxybetaine methacrylate) (pCBMA) and 280 poly(sulfobetaine methacrylate) pSBMA-modified silica prisms 281 using SFG (Figure 4) at different solution $\mathrm{pH}$ values showing 282 that, depending on $\mathrm{pH}$-induced protonation/deprotonation of 283 the zwitterionic groups, the water molecules adopt different 284 orientations. $^{76}$ This work thus provides important insights on 285 the relationship between nonfouling behavior and the external 286 environment (such as $\mathrm{pH}$ ), which might be applied in $\mathrm{pH}-287$ responsive nonfouling interfaces. Further studies of the 288 hydration of SBMA as well as acrylamide and polysaccharide 289 
A

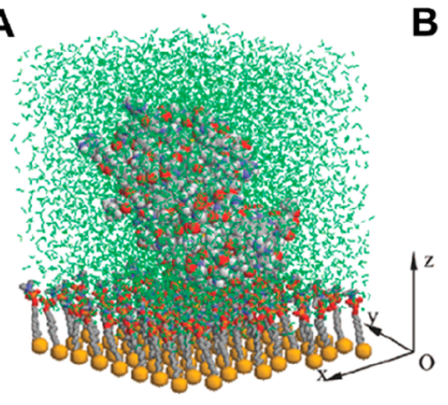

B

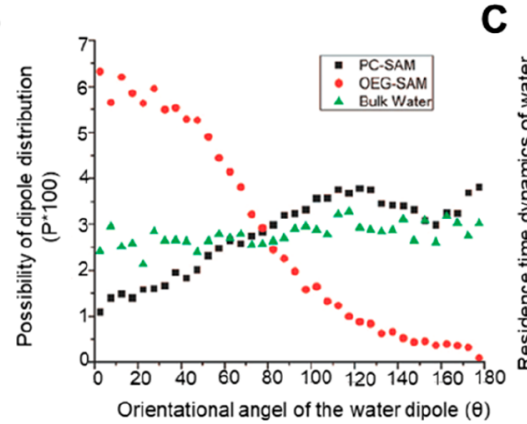

C

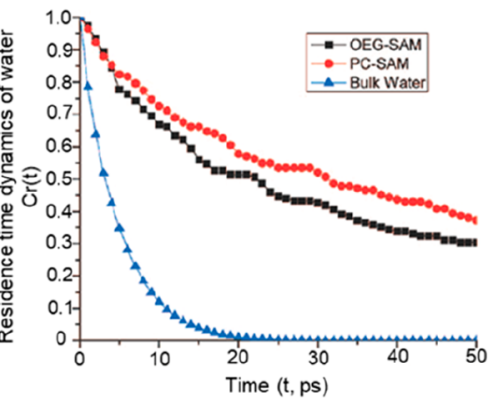

Figure 5. Simulation of water structure at PC and OEG SAMs. (A) Simulated structure, including water, of PC SAMs and model protein (lysozyme) (B) Water dipole distribution near the PC and OEG-SAM and in bulk water and (C) Water residence times of SAMs and bulk water. Adapted with permission from ref 81. Copyright 2008 American Chemical Society.

290 (alginate and hyaluronic acid) based polymers have further 291 reinforced the stronger hydrogen-bonding water association 292 with SB in comparison to the neutral hydrophilic polymers. ${ }^{77}$ 293 Recently, the same group used SFG vibrational spectroscopy to 294 study the interfacial water structure of mixed charged polymers 295 generated by surface initiated-ATRP (SI-ATRP) on a silica 296 prism revealing that only the net neutral 1:1 mixed charge 297 interface shows significant antifouling properties in single 298 protein solutions, reinforcing the importance of high hydro299 philicity and low net charge (polymer thickness: $20 \mathrm{~nm}$ ). ${ }^{78} \mathrm{In}$ 300 summary, we have a picture, then, that surface hydration, as 301 can be assessed by SFG vibrational spectroscopy, has a large 302 impact on the subsequently resolved antifouling performance 303 of the interface.

304 2.2.1.2. Molecular Simulations. Computational studies are 305 valuable in both supporting these experimental observations 306 and in potentially aiding interfacial design without the need for 307 prior acquisition of specific data. In 1991, Jeon et al. reported 308 the first theoretical study of protein adsorption onto PEG309 decorated interfaces. They noted that steric repulsion resulting 310 from the compression of PEG chains upon approach of the 311 protein to the surface was the main driving force preventing 312 adsorption. It was concluded that longer chains and higher 313 surface densities would lead to better protein resistance. ${ }^{79}$ 314 However, in this study no atomic-level information of 315 hydration was considered. To directly correlate film hydration 316 with antifouling performance, Zheng et al. have reported a 317 restrained molecular dynamics (MD) simulation to calculate 318 the interaction forces exerted onto a protein from an OEG319 SAM and its associated water, as the protein approaches the 320 surface. Their force-distance profiles show that the strength of 321 repulsive force acting on the protein decreases across OEG322 SAMs > OH-SAMs > $\mathrm{CH}_{3}$-SAMs. Moreover, this work 323 appeared to confirm that the total repulsive force is composed 324 of two contributions: one from the SAM surface (loss of 325 conformational entropy) and the other from interfacial water 326 with the latter contributing more. ${ }^{80}$

327 In another study, $\mathrm{He}$ et al. compared the structural and 328 dynamic properties of water molecules near the SAMs of 329 zwitterionic PC and nonionic EG using MD simulations 330 (Figure 5A). They showed that water molecules near the PC331 SAMs have a lower mobility, wider dipole orientation 332 distribution and residence time than those near the EG333 SAMs (Figure 5B,C). ${ }^{81}$ In another paper, Shao et al. calculated 334 the hydration free energies of $\mathrm{CB}, \mathrm{SB}$, and $\mathrm{EG}_{4}$ moieties in 335 SAMs using the free energy perturbation method. ${ }^{82}$ The 336 hydration free energy of zwitterionic CB and SB moieties was observed to be much larger $\left(-404\right.$ and $-519 \mathrm{~kJ} \mathrm{~mol}^{-1}, 337$ respectively) than that of nonionic $\mathrm{EG}_{4}$ moieties (-182 kJ 338 $\mathrm{mol}^{-1}$ ), indicating that, as fully expected and noted already, 339 zwitterionic materials exhibit stronger hydration. ${ }^{61}$ In a recent 340 study the difference in self-association between oppositely 341 charged moieties in zwitterionic pSBMA and PCBMA was 342 investigated using $\mathrm{MD}$ simulations (at $1.5 \mathrm{M}$ in solution). ${ }^{83} 343$ Results indicated more and stronger interactions (associations) 344 between the oppositely charged groups in pSBMA due to the 345 similar charge density of the sulfonate and ammonium 346 moieties. In contrast, the larger discrepancy in charge density 347 of carboxylate and ammonium in pCBMA leads, apparently, to 348 fewer/weaker interactions but facilitates a stronger hydration. 349

When nonfouling interfaces are applied within field-effect 350 transistors (FETs) or other electroanalytical configurations, the 351 influence of field on molecular orientation is worthy of 352 consideration. In MD simulation studies by Xie et al. PC-SAMs 353 were found to be electrically responsive such that protein- 354 resistance could be controllably modulated. ${ }^{84}$ Results specif- 355 ically indicated that PC-SAMs can exhibit three states with 356 different charge distributions, namely, one where both 357 negatively charged phosphate groups and positively charged 358 choline groups are exposed to solution (state 1, no electric 359 field), only the phosphate groups are exposed with the choline 360 groups buried (state 2, positive electric field), and choline 361 groups exposed to the solution when the PC chains are 362 stretched (state 3, negative electric field). As a result, the 363 interaction between the model protein cytochrome c (iso- 364 electric point of 9.6) and the surface was deemed tunable. In 365 state 1 , adsorption was predicted to be reversible, while in 366 states 2 and 3, adsorption was enhanced and retarded, 367 respectively, due to electrostatic interactions between the 368 positively charged protein and the exposed groups. Such 369 findings potentially enable an enhancement of antifouling 370 properties by tuning the external electric field and provide a 371 note of caution in assuming native characteristics are retained 372 when surface-imposed fields are actioned.

The presented computational studies, then, support the 374 picture developed by spectroscopic analysis that a strongly 375 bound hydration layer plays a pivotal role in preventing 376 interfacial fouling and offer a fundamental view into the 377 behavior of antifouling molecular systems. It is sensible to note 378 that current simulation studies are both limited in chemical 379 scope and usually conducted with model protein solutions; 380 they do not offer a full picture on the real-world behavior of 381 the antifouling interfaces. For example, the impact of the 382 antifouling components on specific binding events at 383 
A

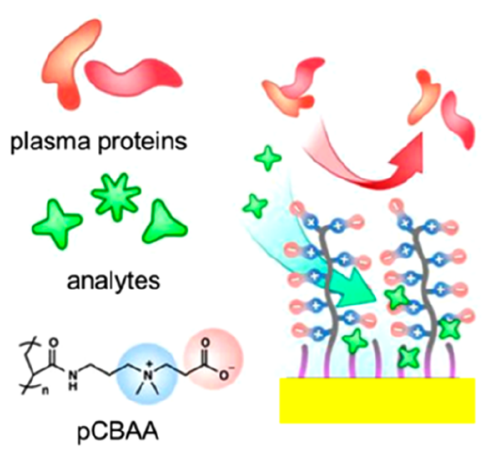

B

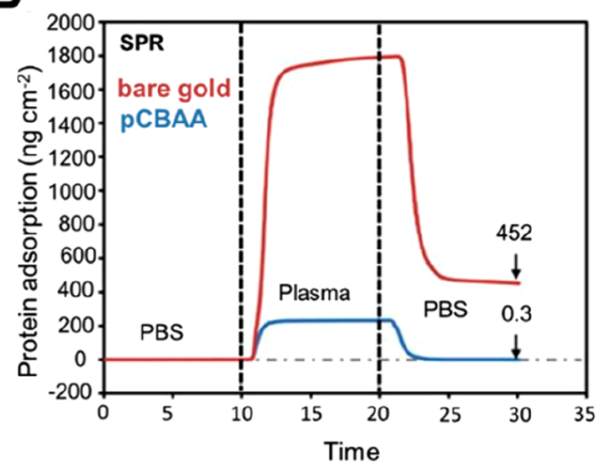

Figure 6. (A) Schematic of a hierarchical pCBAA-based zwitterionic nonfouling modification on a gold surface. (B) Typical SPR sensogram of protein adsorption from undiluted plasma on bare gold and a pCBAA-modified gold surface. Reproduced with permission from ref 87 . Copyright 2016 Springer Nature.

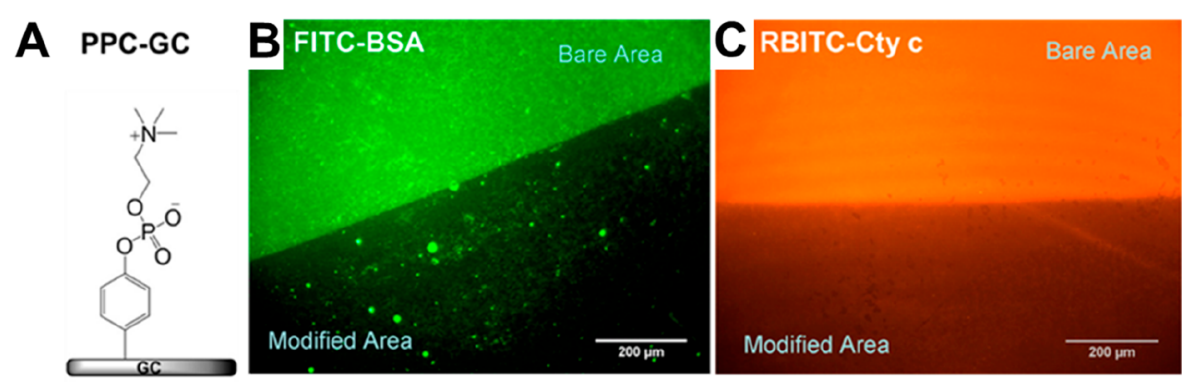

Figure 7. (A) Chemical structure of PPC on glassy carbon electrode. Evaluation of protein adsorption of (B) FITC-BSA and (C) RBITC-Cyt on PPC-GC surface by fluorescence imaging. Scale bars are $200 \mu \mathrm{m}$. Reproduced with permission from ref 88 . Copyright 2013 American Chemical Society.

384 incorporated bioreceptors has not been investigated at any 385 depth nor have analyses been carried out to model more 386 complex solutions where a broad range of intermolecular 387 interactions are present. This gap in data will undoubtedly 388 close with increasing computational power.

389 2.2.2. Evaluation of Antifouling Behavior. In seeking to 390 apply some of the above principles in an analytical format that 391 additionally enables selective target recruitment, careful 392 component selection needs to be married to a prior (chemical, 393 physical, spectroscopic) analysis of the nonfouling interface. In 394 this section, we focus on the experimental methods that have 395 been routinely used to examine interactions at functionalized 396 solid surfaces (e.g., flat electrodes, spherical particles).

397 2.2.2.1. Surface Plasmon Resonance. Surface Plasmon 398 Resonance (SPR) has been extensively used to examine 399 protein adsorption at chemically decorated interfaces, enabling 400 a real time and quantitative probing without the necessity for 401 labeling. SPR takes advantage of surface plasmons generated by 402 incident light excitation in thin metal layers. It is specifically 403 sensitive to dielectric constant change above the metal layer 404 and can thus report on binding events (including kinetics). ${ }^{85}$ 405 This enables a facile evaluation of specific and nonspecific 406 adsorption events and has thus been heavily utilized not only 407 for biosensing but also evaluation of protein adsorption at 408 PEG, ${ }^{11,12}$ zwitterion $^{15-17}$ or peptide-based $^{20}$ antifouling 409 interfaces. For example, a direct comparison of fibrinogen 410 adsorption on five SAMs (OEG, PC, oligo-PC and mixed411 charge SAMs) as well as three polymer brushes (pCBMA, 412 pSBMA, pOEGMA) on gold has been reported. ${ }^{86}$ All 413 interfaces exhibit a low fibrinogen adsorption (from single 414 protein solution, $1 \mathrm{mg} \mathrm{mL}^{-1}$ ); $3.8 \mathrm{ng} \mathrm{cm}^{-2}$ for PC SAMs, and
$<0.3 \mathrm{ng} \mathrm{cm}{ }^{-2}$ for the other surfaces), but adsorption from 415 undiluted human blood plasma was observed to vary widely, 416 with polymer brushes exhibiting fouling levels some 25-30 417 times lower than the SAMs and pCBMA having the lowest 418 levels of total protein adsorption $\left(0.4 \mathrm{ng} \mathrm{cm}^{-2}\right)$. Moreover, 419 decreasing the number of EG or PC units in the SAMs was 420 observed to lead to an expected increase in adsorption from 421 both fibrinogen solution and plasma (see also Section 3.1.2). ${ }^{86}{ }_{422}$ As shown in Figure 6, very low fouling levels from undiluted 423 f6 plasma, assessed by SPR, has also recently been reported for a 424 gold substrate decorated with SAMs of pCBAA $\left(0.3 \mathrm{ng} \mathrm{cm}^{-2} 425\right.$ vs $452 \mathrm{ng} \mathrm{cm}^{-2}$ for bare gold). ${ }^{87}$

2.2.2.2. Fluorescence Imaging. Fluorescence imaging offers 427 a very convenient and accessible way of examining the spatial 428 distribution and status (such as aggregation) of adsorbed 429 fluorophore-labeled materials such as proteins or cells and can 430 thereby directly report on interfacial fouling. ${ }^{8-91}$ The 431 Gooding group, for example, have employed fluorescence 432 imaging to compare protein adsorption levels on glassy carbon 433 (GC) and gold surfaces modified with zwitterionic phenyl- 434 phosphoryl choline (PPC) $)^{88,91}$ using fluorescein isothiocyanate 435 labeled BSA (FITC-BSA) and rhodamine B labeled 436 cytochrome c (RBITC-Cyt c) (Figure 7). BSA and Cyt c $437 \mathrm{f7}$ were chosen as representative anionic and cationic proteins at 438 physiological $\mathrm{pH}$ and it was demonstrated that the protein- 439 resistance of electrodeposited zwitterionic PPC was superior to 440 that of electrodeposited OEG and bare surfaces with a 441 reduction of protein adsorption of $83 \%$ and $90 \%$, respectively. 442 In other work, Zhang et al. modified planar gold with a SI- 443 ATRP generated polymer containing pHEMA and a 444 HWRGWVA peptide (an immunoglobulin G (IgG) recep- 445 
446 tor). ${ }^{92}$ Fluorescence experiments then showed that FITC-IgG 447 did not foul on a pHEMA control but was specifically recruited 448 to the pHEMA-receptor interface.

449 Similarly, Cao et al. have applied fluorescence imaging to 450 evaluate cell adhesion on poly(sulfobetaine-3,4-ethylenediox451 ythiophene) (pSB-EDOT) coated gold surface. ${ }^{93}$ Adhered 452 cells were directly stained with fluorescein diacetate showing 453 negligible cell attachment on pSB-EDOT while the unmodified 454 poly(3,4-ethylenedioxythiophene) (PEDOT) control showed 455 significant cell fouling. While offering simple visual, qualitative 456 assessment of fouling it should be taken into consideration that 457 fluorescence imaging requires the conjugation (and subsequent 458 purification) of suitable fluorophores onto the model fouling 459 agent (e.g., protein) which can be cumbersome and not readily 460 extendable to a refined analysis of complex samples containing 461 a broad range of different proteins.

462 2.2.2.3. Quartz Crystal Microbalance with Dissipation. A 463 Quartz Crystal Microbalance with Dissipation (QCM-D) 464 constitutes another sensitive label-free technique, in which an 465 alternating voltage is applied to drive the oscillation of a quartz 466 substrate at its resonance frequency. ${ }^{94}$ Relevant parameters, 467 such as the mass of adsorbed protein, including associated 468 solvent molecules and the viscoelastic properties of the 469 adsorbed layer, can be determined from frequency $(\Delta f)$ and 470 dissipation change $(\Delta D)$. A combination of the two factors can 471 be used to determine the interaction between underlying 472 nonfouling materials and potential adsorbates. ${ }^{95-97}$

473 Utilizing QCM-D, the protein resistance of simple 474 zwitterionic SAMs of L-cysteine, L-methionine, and glutathio475 nine (GSH) has, for example, been evaluated. ${ }^{94}$ The data 476 demonstrated that, of these interfaces, the GSH-SAM showed 477 lowest fouling when exposed to $1 \mathrm{mg} \mathrm{mL}^{-1}$ BSA at 478 physiological $\mathrm{pH}\left(37 \mathrm{ng} \mathrm{cm}{ }^{-2}\right.$ vs $749 \mathrm{ng} \mathrm{cm}^{-2}$ for bare $\mathrm{Au}$ ) 479 and that the fouling of all SAMs was predictably $\mathrm{pH}$ 480 dependent. For example, the GSH film showed high fouling 481 at $\mathrm{pH} 4.0$ and relatively low fouling at $\mathrm{pH}$ 5.0. This is due to 482 electrostatic attraction between BSA and $\mathrm{GSH}$ at $\mathrm{pH} 4.0$, due 483 to the pI of BSA and immobilized GSH of 4.7 and 3.9, 484 respectively; they are oppositely charged at this $\mathrm{pH}$. This nicely 485 highlights the attention that should be given to the $\mathrm{pH}$ 486 dependence of nonspecific electrostatic associations. Recently, $487 \mathrm{Li}$ et al. investigated the construction of an effective antifouling 488 layer via electrodeposition. ${ }^{98}$ The gold electrode was modified 489 with zwitterionic SB via diazonium chemistry and fouling 490 studies to $10 \%$ fetal bovine serum (FBS) carried out by QCM$491 \mathrm{D}$. The authors showed that this film possessed similar 492 antifouling capabilities as the analogous SB-SAM.

493 In another study, QCM was used to evaluate the antifouling 494 performance of zwitterionic peptides immobilized on polydop495 amine (PDA)-treated gold substrates. ${ }^{99}$ The alternating 496 glutamic acid- and lysine-based peptide interface was observed 497 to show adequate fouling resistance $\left(\sim 110 \mathrm{ng} \mathrm{cm}^{-2}\right)$ to human 498 serum, significantly better than bare gold $\left(\sim 700 \mathrm{ng} \mathrm{cm}^{-2}\right)$, $499 \mathrm{PDA} / \mathrm{Au}\left(\sim 870 \mathrm{ng} \mathrm{\textrm {cm } ^ { - 2 }}\right)$ and $\mathrm{PEG} / \mathrm{PDA} / \mathrm{Au}(\sim 270 \mathrm{ng}$ $\left.500 \mathrm{~cm}^{-2}\right)$.

501 2.2.2.4. Electrochemical Impedance Spectroscopy. Elec502 trochemical impedance spectroscopy (EIS) is a technique that 503 can be applied to the characterization of electrode interfaces 504 and sensitively report on interfacial change such as those 505 associated with binding events (specific or nonspecific). ${ }^{100}$ In 506 recent work from Jiang and co-workers, EIS was applied to 507 evaluate the interactions between proteins and zwitterionic 508 PPC-modified indium tin oxide (ITO) electrodes, in which
HSA was selected as a nonspecific model protein. ${ }^{36}$ Upon 509 exposure to HSA $\left(1 \mathrm{mg} \mathrm{mL}^{-1}\right)$ the charge transfer resistance 510 $\left(R_{\mathrm{ct}}\right)$ of the interface did not change, indicating negligible 511 fouling. Similar results were also obtained by Bryan et al. at 512 PEG SAMs. ${ }^{47}$ Davis and co-workers have applied non-Faradaic 513 EIS to evaluate protein adsorption on cysteamine/graphene 514 oxide-modified gold microelectrodes. ${ }^{101}$ They found that after 515 $30 \mathrm{~min}$ exposure to $100 \%$ serum or $1 \mu \mathrm{M}$ BSA in phosphate 516 buffered saline (PBS) for $30 \mathrm{~min}$, the relative change in 517 impedimetric modulus $(\mathrm{Z})$ is considerably lower for cyste- 518 amine/GO composite electrodes $(<1 \%)$ than that of simple 519 cysteamine SAMs $(\sim 24 \%)$, indicating a significant contribu- 520 tion of GO to overall antifouling performance. Non-Faradaic 521 EIS has also been applied to the ultrasensitive quantification of 522 insulin in neat serum using pCBMA-modified gold electrodes 523 (see also Section 2.3.2). ${ }^{102}$ Similarly, the fouling of HSA ( 1524 $\mathrm{mM}$ ) on ferrocene-tagged peptide SAMs has been evaluated by 525 impedance-derived redox-capacitance. ${ }^{103}$ In other examples, 526 Luo's group have assessed the antifouling performance of 527 sensors based on PEG-aptamer-modified PDA/GC ${ }^{104}$ and 528 zwitterionic peptide (EKEKEKE-PPPPC)-modified gold elec- 529 trodes $^{33,105}$ using Faradaic EIS, indicating negligible protein 530 adsorption from single protein solution and in diluted plasma 531 in both cases.

2.2.2.5. Dynamic Light Scattering. Through appropriate 533 decoration with antifouling layers (nano)particle-based plat- 534 forms have gain increasingly intense levels of attention as 535 promising diagnostic and therapeutic tools. ${ }^{106}$ In this context, 536 dynamic light scattering (DLS) is a useful technique not only 537 for determination of particle size and size distribution but also 538 for examination of subsequent fouling/absorption or aggrega- 539 tion events. Upon fouling at the liquid-solid interface, a 540 change in the hydrodynamic diameter $\left(D_{\mathrm{h}}\right)$ of the particle can 541 be measured. Wang et al. have, for example, reported an 542 analysis of zwitterion functionalized spherical nanoparticles 543 (size). ${ }^{107}$ Specifically, silica nanoparticles were conjugated with 544 natural amino acids (L-lysine, L-cysteine, L-arginine) and their 545 antifouling properties indirectly evaluated in BSA and FBS 546 solution, whereby protein adsorption is accompanied with an 547 increase in $D_{\mathrm{h}}$. It was shown that all of the tested amino acid- 548 functionalized silica nanoparticles can effectively resist protein 549 adsorption under these conditions. Among them, lysine- 550 functionalized silica nanoparticles had the best antifouling 551 performance, showing an increase of $\left(D_{\mathrm{h}}\right)$ of only $10 \%$ after 552 incubation for up to $24 \mathrm{~h}$ in $1 \mathrm{mg} \mathrm{mL}^{-1}$ BSA solution and $20 \% 553$ after incubation in $10 \%$ FBS solution (initial $D_{\mathrm{h}} \approx 65 \mathrm{~nm}$ ). In 554 another paper reported by Sanchez-Salcedo et al. the same 555 approach was applied to evaluate the antifouling performance 556 of zwitterionic 2-methacryloyl posphorylcholine (MPC)- 557 coated mesoporous silica nanoparticles in $1 \mathrm{mg} \mathrm{mL}^{-1}$ BSA 558 and $10 \%$ FBS solutions. ${ }^{108}$ Results showed that $D_{\mathrm{h}}$ remained 559 stable over $72 \mathrm{~h}$. More recently, the antifouling behavior of 560 silica nanoparticles decorated with different compositions of 561 (3-aminopropyl)triethoxysilane (APTES) and zwitterionic SB- 562 silane was assessed (Figure 8). ${ }^{109}$ After exposure to FBS, only a $563 \mathrm{fo}$ slight increase $(\sim 2-3 \mathrm{~nm})$ in size of the nanoparticles 564 containing large proportions of zwitterionic moieties (100\% 565 SB and $75 \%$ SB) was noted. This technique is readily 566 applicable for the evaluation of well-dispersed and homoge- 567 neous nanoparticles, but limitations arise for larger beads that 568 aggregate or sediment which can severely interfere with the 569 scattering measurements. 


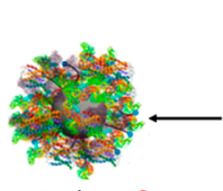

$D_{\mathrm{h}}$ change?
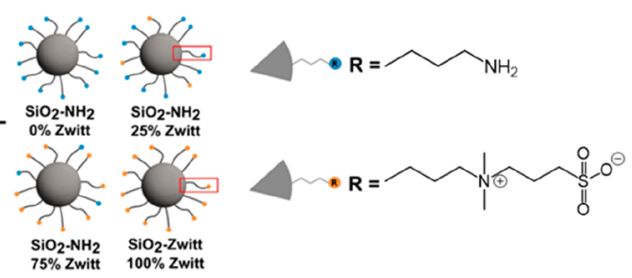

Figure 8. Nanoparticles modified with mixed silanes terminated with amine and zwitterionic SB groups where protein adsorption is evaluated by DLS. Adapted with permission from ref 109. Copyright 2018 American Chemical Society.

571 2.2.2.6. Bicinchoninic Acid Assay. The Bicinchoninic Acid 572 (BCA) assay is a quantitative colorimetric assay for total 573 soluble protein and can be used to indirectly study protein 574 adsorption at a solid-liquid interface by monitoring solution 575 depletion. For example, the protein-resistance of pSBMA576 modified magnetic nanobeads has been assessed this way, ${ }^{110}$ 577 where the bead surface modification was, as expected, shown 578 to dramatically reduced nonspecific BSA adsorption $(<10 \mu \mathrm{g}$ 579 adsorption/mg beads). Similarly, BSA fouling resistance (from 580 single protein solution, $4.5 \mathrm{mg} \mathrm{mL}^{-1}$ ) was demonstrated for 581 PC-coated hydroxyapatite with fouling reduction of $50 \%$ in 582 comparison to the unmodified particles. ${ }^{111}$ Estupiñán et al. 583 recently prepared a magnetic multifunctional silica particle 584 platform containing antifouling moieties (SB) as well as anchor 585 groups for receptor conjugation (amine and alkene terminated 586 silanes). The protein resistance in undiluted FBS was assessed 587 with the BCA assay after particle incubation revealing only 588 moderate $\left(<60 \mathrm{ng} \mathrm{cm}{ }^{-2}\right)$ fouling. Furthermore, it was 589 demonstrated that the presence of SB did not prohibit 590 subsequent controlled conjugation of an IgG antibody. ${ }^{112}$

591 There are then, a number of sensitive interfacial techniques 592 available to allow characterization of antifouling materials and 593 their performance on diverse solid supports (conducting or 594 nonconducting, flat or nanoparticle). Some of these allow a 595 direct quantification of adsorbed mass on the interface (e.g., 596 SPR, QCM) while others can serve as a useful qualitative tool 597 to assess fouling (DLS, electrochemistry, (fluorescence) spectroscopy, etc.). In addition to the highlighted approaches, 598 a number of other techniques such as isothermal titration 599 calorimetry (ITC), ${ }^{113}$ ellipsometry $^{114}$ or differential pulse 600 voltammetry (DPV) ${ }^{115,116}$ can also be readily utilized to 601 investigate surface adsorption events. Through the appropriate 602 selection of these complementary techniques, a detailed 603 evaluation of fouling performance can be entertained through 604 the use of (ideally) realistically complex test solutions. This 605 data can then guide the design of sensing interfaces with 606 improved performance, most notably selectivity and robust- 607 ness.

608

\subsection{Sensors Based on Chemical Antifouling Interfaces}

2.3.1. Incorporation of Receptive Elements. In any 609 specific downstream sensory application, functional (bio)- 610 recognition elements need to be incorporated into antifouling 611 surface architectures. It is, of course, imperative (or at least 612 ideal) that the performance of these additional functionalities 613 is not impeded by the nonfouling support and, equally, that the 614 incorporation of significant amounts of such elements can be 615 carried out with minimal perturbation of the supporting 616 antifouling performance. ${ }^{30}$ Therefore, the appropriate (co)- 617 incorporation of receptor/anchor and antifouling units is a 618 crucial part of the sensor design. If both functionalities are 619 inherently present in one component such as $\mathrm{CB}$ or peptides 620 or functional group terminated OEGs, ${ }^{34}$ then direct assembly 621 of this component will yield a modifiable interface in one step 622 (Figure 9A). If the antifouling component is not easily $623 \mathrm{fg}$ functionalizable, like phosphorylcholine or sulfobetaine, then 624 an additional anchor component must be immobilized 625 simultaneously or consecutively to form a mixed interface 626 (Figure 9B). This can usually be achieved by, for example, self- 627 assembly, electrografting or copolymerization. Importantly, if 628 (bio)recognition and nonfouling elements are coimmobilized 629 their surface ratio must be tuned to obtain the best sensor 630 performance in terms of antifouling performance, target 631 capture efficacy and interfacial selectivity. Depending on the 632 specific tethering method, and the relative reactivity of the 633 different components, this tuning can usually be carried out by 634

A
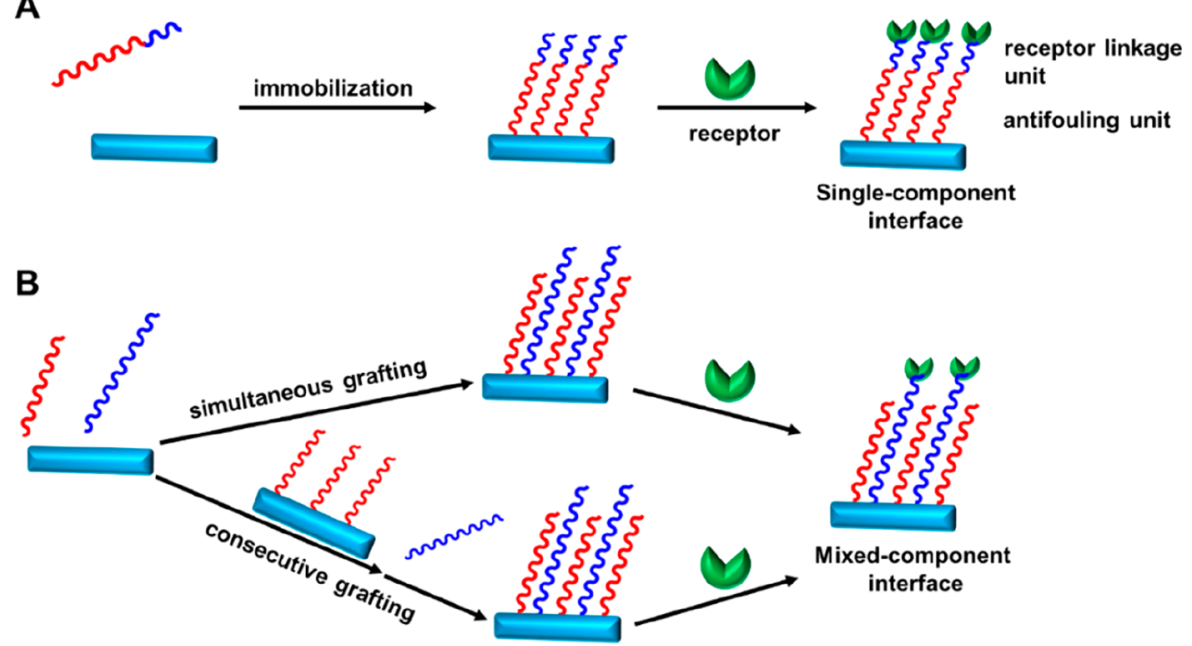

Figure 9. General construction principle of antifouling sensors with anchor sites for receptor immobilization. (A) Antifouling unit (orange) and anchor site for (bio)receptor conjugation (blue) are present in the same component and can thus be assembled in one step to form a "homogeneous" film. (B) Antifouling and anchor units are immobilized separately by either simultaneous or consecutive grafting to form a "heterogeneous" sensory interface. 
635 adjusting the immobilization protocol (e.g., concentration, 636 time). ${ }^{56}$

637 In a typical example, as shown in Figure 10, a mixture of 638 functional elements can also be immobilized by electrografting.

A

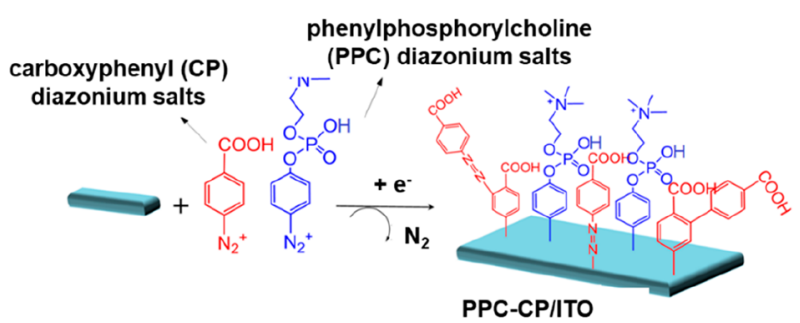

B
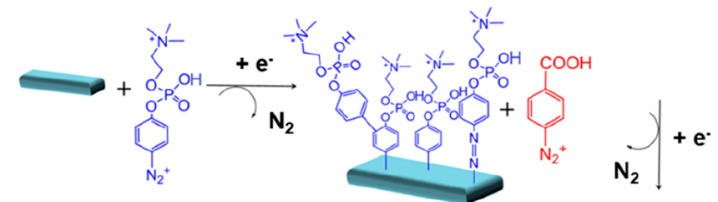

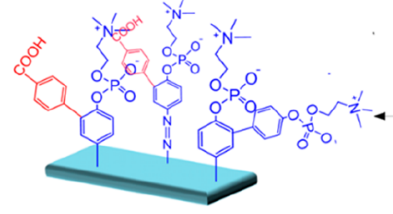

PPC-CP/ITO (pH=7.4)

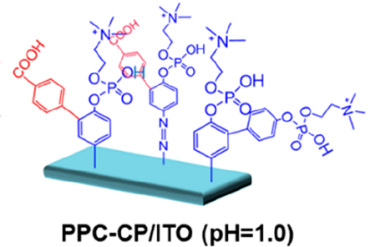

PPC-CP/ITO (pH=1.0)
Figure 10. Electrografting mixed layers of PPC and CP on ITO using (A) simultaneous electrografting strategy and (B) consecutive electrografting strategy. Reproduced with permission from ref 36. Copyright 2016 American Chemical Society.

639 For example, simultaneous and consecutive electrografting of 640 zwittterionic PPC diazonium salts with carboxyphenyl 641 diazonium salts onto ITO electrodes has been investigated 642 by Jiang et al. ${ }^{36,54}$ The authors demonstrated that, with the 643 employment of consecutive electrografting, the surface ratio of 644 mixed films can be tuned and antifouling abilities retained 645 through the conjugation of high levels of bioreceptive elements 646 (anti-TNF- $\alpha$ antibody).

647 Regardless of the specific immobilization protocol that is 648 utilized, attention must be given to the compatibility of all 649 immobilized anchor/receptive/nonfouling components. This 650 includes, but is not limited to, their stability, reactivity, and 651 spatial arrangement. The latter is particularly important, where 652 care must be taken to ensure that the integration of any 653 antifouling components does not (significantly) affect the 654 operation of the sensor (e.g., signal transduction and binding 655 selectivity). For example, when receptive elements are 656 incorporated into the interface, the nonfouling support should 657 not sterically (or electrostatically) impede target binding nor 658 present a chemical environment that is otherwise not 659 conducive to binding (such as a particularly high dielectric 660 where target binding has a large electrostatic component). A 661 sterically imposed "filtering" of background can be beneficial in 662 very specific cases (see Sections 3 and 5). ${ }^{117}$ One notable 663 exception to these steric considerations is when receptive units 664 are temporarily embedded within the antifouling interface and 665 controllably exposed upon the application of a stimulus (e.g., 666 electric field, temperature, light). ${ }^{118-120}$

667 Another important consideration is the effect receptor 668 coupling has on the underlying "native" fouling characteristics of the supporting film; the commonly utilized tethering to film 669 exposed anionic carboxyls, for example, will render an initially 670 zwitterionic moiety cationic. In addition to both the effect of 671 nonfouling chemistry on target capture and the potential 672 impact of receptor integration on nonfouling character, one 673 most also consider signal transduction. If, for example, 674 comparatively thick nonfouling films are married with low 675 levels of receptor; one may then be in a regime where target 676 capture is not effectively transduced to an underlying mass, 677 optical, or electrochemical sensor.

678

On the basis of the above analyses and general design 679 principles, a broad range of electrochemical or optical sensors 680 for a variety of analytes have been constructed and utilized in 681 vitro and in vivo. These examples will be discussed in the 682 following sections. It should be noted (again) that label-free 683 approaches are significantly more sensitive to nonspecific 684 adsorption because they cannot natively distinguish non- 685 specific from specific binding events and thus almost inevitably 686 require the integration of antifouling approaches when sensing 687 in complex biological media is sought. Sensors based on 688 multistep labeled-designs (e.g., enzyme-linked immunosorbent 689 assay (ELISA)) are less prone to this nonspecificity (though it 690 undoubtedly remains) and very often contain no predesigned 691 antifouling components; they are thus not the main focus 692 herein.

693

2.3.2. In Vitro Sensing Based on Antifouling Chem- 694 istries. Optical or electrochemical sensor formats have long 695 been the most commonly used in applications in complex 696 biological fluids. In the following sections, we will highlight 697 relevant recent examples of such sensors and discuss their 698 analytical performance. We will specifically focus on those 699 presenting novel architectures capable of operating in real-life 700 samples such as serum.

2.3.2.1. PEG. Since the 1970 s, polyethylene glycol (PEG; 702 $>10$ EG units) and its derivatives such as oligoethylene glycol 703 (OEG, $n \approx 3-10$ ) have undoubtedly been the most accessible 704 and frequently applied antifouling materials ${ }^{121}$ and have been 705 utilized in numerous sensory formats. Particularly common are 706 sensors based on PEG-based SAMs such as a recently 707 developed multiplex SPR sensor for the simultaneous detection 708 of three human pancreatic islets peptide hormones, that is, 709 insulin, glucagon, and somatostatin. ${ }^{122}$ In this work, a mixed 710 $\mathrm{SAM}$ of $\mathrm{CH}_{3} \mathrm{O}$-PEG-SH and 16-mercaptohexadecanoic acid 711 (molar ratio of 3:2) was utilized as an antifouling support and 712 for antibody attachment, respectively. Antibody functionalized 713 spots on the sensor chips showed high specificity to the 714 corresponding hormones with negligible interference from 1715 $\mathrm{mg} \mathrm{mL} \mathrm{mL}^{-1} \mathrm{BSA}$ or lyzosome and adequate detection limits for 716 insulin $(1 \mathrm{nM})$, glucagon $(4 \mathrm{nM})$, and somatostatin $(246 \mathrm{nM}) \cdot 717$ While it was not demonstrated that this sensor is capable of 718 operating in real-life samples, the multiplexed detection of 719 different markers with high throughput is of large interest in 720 clinical applications. In another study, $\mathrm{Xu}$ et al. reported a 721 label-free impedimetric sensor to detect insulin using a simple 722 SAM of SH-(EG) ${ }_{4}-\mathrm{COOH}$ as simultaneous antifouling layer 723 and antibody anchoring support. ${ }^{123}$ They demonstrated that 724 this sensor is capable of insulin detection in 50\% serum with 725 minimal interference (3\%), a limit of detection (LoD) of 1.2726 pM, and a clinically relevant linear range (from 5 pM to 50727 nM). Notably, the obtained sensor could be regenerated and 728 reused by disassociation of insulin antibody-antigen complex 729 using 0.2 M Gly-HCl buffer. 

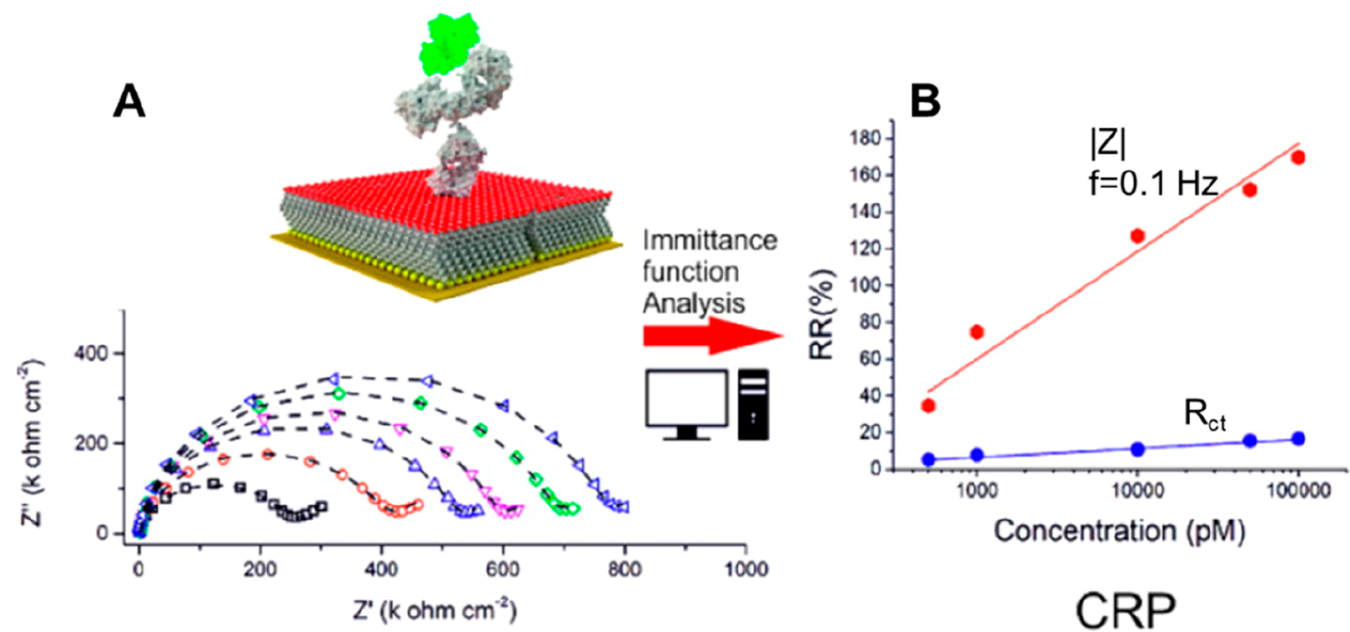

Figure 11. (A) PEG thiol (HS-C11-(EG) $3-\mathrm{OCH}_{2}-\mathrm{COOH}$ ) based interface for label-free immittance electroanalysis of CRP. (B) Optimized analytical response curve for impedimetric CRP detection showing that IZI is a $12 \times$ more sensitive analytical parameter than the more commonly used Rct. Adapted with permission from ref 125. Copyright 2015 American Chemical Society.

731 Other impedimetric sensors based on PEG chemistries have 732 also been reported by the Davis group. For example, $\alpha$ 733 synuclein antibodies (markers for Parkinson's disease, PD) 734 have been quantified in blood at a $\alpha$-synuclein antigen735 modified SAM of $\mathrm{HS}-\mathrm{C}_{11}-(\mathrm{EG})_{3}-\mathrm{OCH}_{2}-\mathrm{COOH}$ (EG-SAM) 736 on gold. ${ }^{47,124}$ This format was then applied to serum samples 737 of Parkinson's disease (PD) patients and healthy controls 738 demonstrating $\mathrm{pM}$ levels of sensitivity and good levels of 739 disease-relevant selectivity. Similar sensors for the detection of $740 \mathrm{C}$-reactive protein (CRP, an important marker of potential 741 cardiac stress and inflammation) at anti-CRP modified the EG742 SAMs using impedance based electrochemical capacitance 743 spectroscopy (ECS) have also been developed, where an 744 immittance function approach has been employed to extract 745 the most sensitive analytical parameter (Figure 11). ${ }^{125-128}$ 746 Recently, the same group has applied EIS at EG-SAMs on gold 747 bead electrodes to assay the exosomal proteins CD 9 and 748 Syntenin with picomolar sensitivity in exosomal lysate. ${ }^{129}$ 749 Mixed SAMs of EG-thiol and ferrocene-thiol have also been 750 utilized as supports for the redox capacitive detection of 751 nonstructural protein 1 (NS1), IgG, or human prostatic acid 752 phosphatase (PAP) in $20 \%$ serum. ${ }^{130}$ The multiplexed 753 impedimetric sensing of insulin and CRP has also been 754 reported at PEG-modified gold electrode arrays. Specifically 4755 armed PEG-amine and 4-armed PEG-epoxide were phys756 isorbed onto the electrode surface and then thermally cross757 linked prior to antibody tethering. This platform was capable 758 of the detection of insulin and CRP in undiluted human serum 759 with $\mathrm{fM}$ and $\mathrm{pM}$ detection limits respectively, a capability then 760 applied to real patient sample analysis. ${ }^{131}$

761 The Gooding group has investigated antifouling electro762 chemical sensors based on mixed layers of electrografted OEG 763 and molecular wires (MWs). It is worth noting here that while 764 OEG can effectively resist nonspecific protein adsorption, it 765 can, if densely packed, significantly hinder electron transfer to 766 the underlying electrode impeding its use in various electro767 chemical sensors. Thus, additional oligo(phenylethynylene) 768 based MWs were used to switch on the electrochemical 769 communication between an immobilized redox probe 770 (ferrocene) and the electrode. ${ }^{55,132}$ To apply this design, 771 they further incorporated an epitope of biotin into the wire to 772 allow amperometric biotin sensing via a displacement assay, taking advantage of the difference in the affinity of biotin to the 773 antibiotin antibody or its synthetic epitope (Figure 12). Five $774 \mathrm{f} 12$

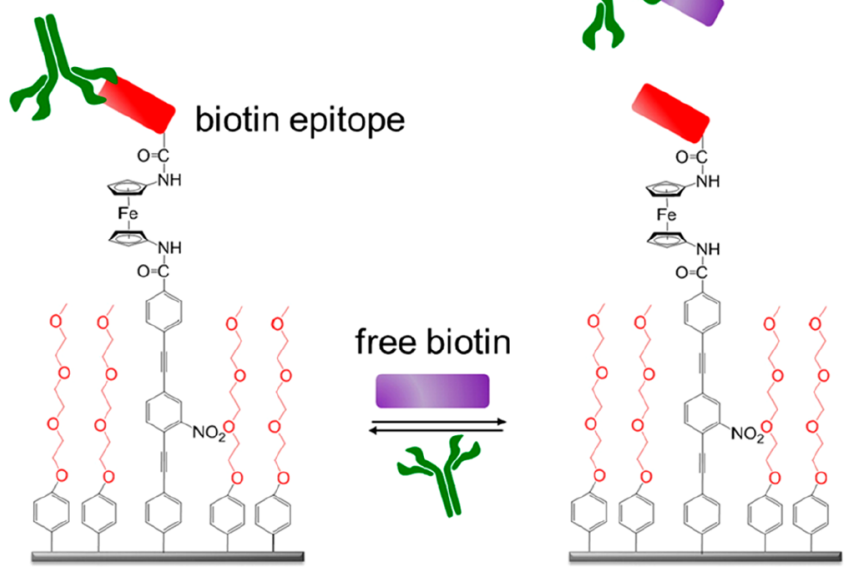

Figure 12. Schematic representation of a mixed OEG/molecular-wire based electrochemical biosensor for detection of biotin. Adapted with permission from ref 54. Copyright 2011 Elsevier.

different combinations of MWs/OEG (1:0, 1:20, 1:50, 1:75, 775 and 1:100) were tested in the detection of the model analyte 776 (biotin) whereby the ratio of 1:50 was found to give the 777 highest sensitivity. This OEG/MW mixed-layer platform was 778 then successfully applied to detect glycosylated hemoglobin 779 (HbAlc) in undiluted human serum by incorporating a 780 recognition epitope (an $\mathrm{N}$-glycosylated pentapeptide) into the 781 wire. $^{55,132}$ A good linear relationship between the relative 782 Faradaic current associated with ferrocene and the concen- 783 tration of $\mathrm{HbAlc}$ from $4.5 \%$ to $15.1 \%$ of total hemoglobin in 784 serum without the need for washing or rinsing steps was 785 achieved, indicating its applicability for point-of-care (PoC) 786 use. ${ }^{133}$ The major limitation of these systems is the relative 787 complexity of the MW synthesis.

788

The Luo and Davis groups have also developed a series of 789 label-free DNA biosensors based on PEG antifouling chemistry 790 to electrochemically detect the breast cancer susceptibility 791 gene 1 (BRCA1) in human serum. In one example, they drop- 792 


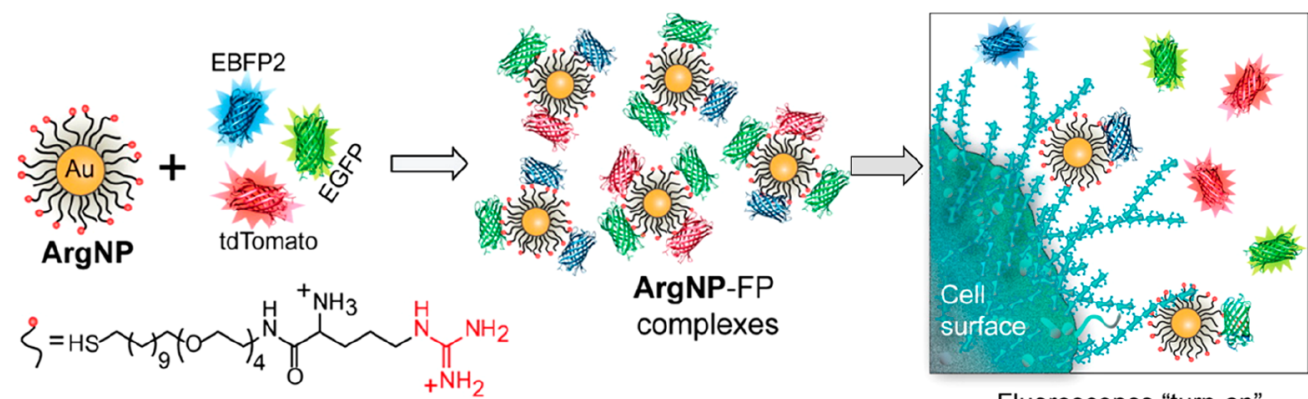

Fluorescence "turn-on"

Figure 13. Fabrication of a fluorescent displacement nanosensor based on the reversible binding of fluorescent proteins (FPs) onto ArgNPs. In binding to the arginine head groups of the PEGylated AuNP, the FP fluorescence is initially quenched by the underlying gold. Displacement by specific cell surface proteins restores their fluorescence. Adapted with permission from ref 137. Copyright 2015 American Chemical Society.

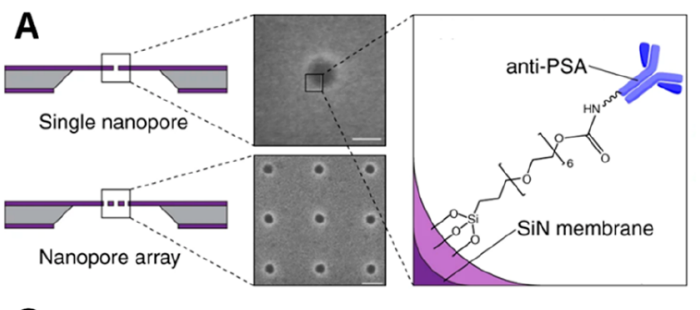

B Nanopore blockade sensing

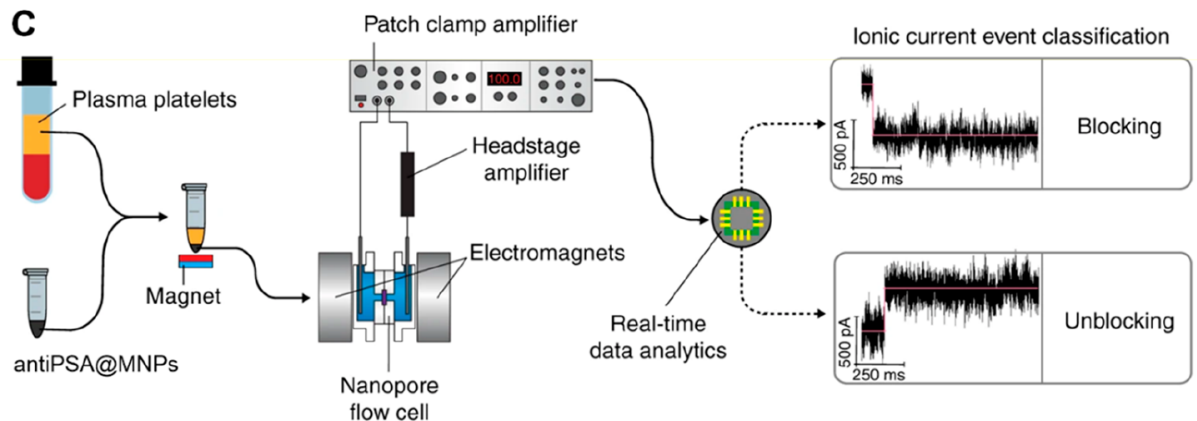

Figure 14. (A) Schematic representation and scanning electron micrographs of a solid-state nanopore modified with silane-EG6-anti-PSA. (B) Removal of nonspecific nanopore blockade events (i.e., anti-PSA-MNP without target) was through the application of a magnet, whereas (antiPSA)-MNP with bound PSA will remain in the pore, thus blocking it. (C) Flowchart for assay of PSA. After incubation and separation of the MNPs, they were exposed to the nanopore flow cell. A potential difference was applied between both sides of the nanopore and the ionic current analyzed by an event classifier where three distinct event types: translocation, blocking, and unblocking can be observed. Adapted with permission from ref 142. Copyright 2019 Nature Publishing Group.

793 coated 4-armed PEG-amine and 4-armed PEG-epoxide onto a 794 GC electrode forming a cross-linked PEG film onto which 795 oligonucleotide probe immobilizing gold nanoparticles were 796 deposited. ${ }^{134}$ Upon binding of the BRCA1 DNA, an increase 797 in the charge transfer resistance of the sensory interface was 798 measured by EIS. The sensor exhibited a three orders linear 799 range with a $\mathrm{fM}$ LoD. Spiking of undiluted serum with target $800(1,10$, and $100 \mathrm{pM})$ showed a recovery rate ranging from $80192.6 \%$ to $100.6 \%$, indicating a robust nonfouling performance. 802 In another study by the same group, a 4-armed-PEG-COOH 803 based nonfouling sensor for BRCA1 electroanalytical detection 804 in serum was developed. ${ }^{38}$ Specifically, polyaniline (PANI) 805 nanowires were introduced onto GC through electropolyme806 rization. Subsequently, this interface was modified with PEG 807 on which the amino functionalized methylene blue-modified 808 DNA capture probes were coupled. Upon target capture, the 809 hybridization of complementary target DNA and DNA probe 810 displaced the MB out of the duplex structure, leading to a 811 reduction in current with a LoD of $0.01 \mathrm{pM}$. Similarly, Chen et 812 al. investigated an antifouling support of PEG/tannic acid/ 813 PDA on GC onto which gold nanoparticles (AuNPs) were electrodeposited to allow attachment of thiolated probe DNA. 814 Impedimetric BRCA1 detection in $10 \%$ serum was achieved 815 with a sub femtomolar LoD. ${ }^{135}$ PEG adlayer methodologies 816 can also be applied to the modification of particulate systems 817 to enable optical based target detection in complex media. For 818 example, Luo's group has investigated PEGylated $\mathrm{Au} / \mathrm{Fe}_{3} \mathrm{O}_{4} 819$ particles for the optical detection of human papilloma virus 820 (HPV) ${ }^{136}$ In this study, the additional decoration of the PEG- 821 based particles with chondroitin sulfate (CSA) resulted in 822 enhanced colloidal stability and endowed the particles with 823 apparent excellent fouling-resistance. A DNA probe for HPV 824 was then immobilized onto the particles. After hybridization 825 (target capture), a fluorescent dye (Hoechst 33258, which 826 exclusively binds to the minor groove of double-stranded 827 DNA) was utilized as a transducer. This fluorescent sensor was 828 observed to possess a wide linear range and a pM LoD. ${ }^{136} 829$

Another fluorescent nanosensor platform has been devel- 830 oped by the Rotello group, using functional PEG-thiol SAMs 831 on small AuNPs ( $2.6 \mathrm{~nm}$ diameter), where the head groups, 832 for example, arginine (Arg), served as noncovalent anchor sites 833 for three different fluorescent proteins (FPs, (EBFP2 (blue), $834 \mathrm{fl} 13$ 
835 EGFP (green), and tdTomato (red)) (Figure 13). ${ }^{137}$ This 836 configuration was then utilized in a displacement assay for the 837 sensing of target analytes that can compete with the FPs for the 838 headgroup binding sites. Depending on the binding properties 839 of the target analytes, the system generates different 840 fluorescence finger prints, which can be analyzed by a linear 841 discrimination assay (LDA). This was then successfully utilized 842 to investigate the physicochemical changes of cell surfaces (as 843 "analyte") upon exposure to chemotherapeutic drugs with 844 unique fluorescent signatures for different response mecha845 nisms. This PEG-based nanoparticle platform was also 846 successfully translated to protein sensing in serum (in addition 847 to bacterial sensing in culture media) by endowing the head 848 groups with specific interaction sites. ${ }^{138-141}$ These particles 849 allowed the high-throughput screening of multiple targets 850 without additional sample preprocessing, thus offering a 851 convenient approach for drug discovery, protein screening, 852 and cell-based sensing.

853 Kyloon et al. utilized OEG-based antifouling chemistry in a 854 silicon nitride nanopore sensor for the sensitive detection of 855 prostate specific antigen (PSA) in blood. ${ }^{142}$ Sensing was 856 carried out by capturing PSA from patient blood with anti-PSA 857 antibody-modified OEG-modified magnetic nanoparticles 858 (MNPs, $50 \mathrm{~nm}$ ). These MNPs were then further captured at 859 anti-PSA antibody-modified photolithographically generated 860 nanopores $(27 \mathrm{~nm})$ whereby the blocking of the pore results in 861 a modified current response (Figure 14). Importantly, the 862 MNPs can be magnetically driven whereby those particles that 863 have not captured the analyte can be magnetically removed, 864 which together with the antifouling OEG modification reduces 865 fouling/nonspecific response. This sandwich format afforded a 866 very high sensitivity (LoD $0.8 \mathrm{fM}$ ) and showcased both the 867 importance of antifouling design and a viable extension to 868 other trace analyte detection. ${ }^{143,144}$ In different work, 869 Giamblanco et al. demonstrated that $(5 \mathrm{kDa})$ PEG-modified $87020 \mathrm{~nm}$ nanopore sensors can be used to study protein 871 aggregate morphology. The intensity of the relative current 872 blockade (aggregate vs translocating) enabled the detection 873 and discrimination of the morphology of B-lactoglobulin, 874 lysozyme, and BSA. ${ }^{145}$

875 Despite its ubiquitous use as an antifouling material, it is 876 important to note that PEG chemistry suffers from a number 877 of major drawbacks such as those associated with chemical 878 tailorability and a noted susceptibility to oxidative dam879 age $^{146,147}$ and decomposition in the presence of oxygen and 880 transition metal ions (impeding long-term stability/function881 ality) (as summarized in Table 4). ${ }^{12,21,148}$ They are also only 882 rarely capable of a prolonged operation in high levels of blood 883 or serum. Under such circumstances, alternatives are required. $884 \quad$ 2.3.2.2. Zwitterions. In recent years, zwitterionic materials, 885 which possess high oxidative resistance and hydrolytic stability, 886 have attracted considerable attention as promising alternatives 887 to PEG in developing high performance antifouling inter888 faces. ${ }^{147,149-151}$ As discussed in Section 2.2, their hydration not 889 only arises from hydrogen-bonding (as is the case for PEG) 890 but also has an appreciable electrostatic component (a key 891 element of their antifouling properties). A range of functional 892 groups can be readily incorporated into zwitterionic surface893 confined architectures; most commonly, quaternary ammo894 nium groups are combined with phosphates/phosphonates 895 (phosphorylcholine), ${ }^{152,153}$ sulfone/sulfate groups (sulfobe896 taine), 154,155 or carboxylates (carboxybetaine) (Figure 897 1B). ${ }^{150,156}$ The latter can furthermore serve as convenient anchor groups for subsequent (bio)conjugation via, for 898 example, esterification or amidation as discussed further. ${ }^{157} 899$

In analogy to the PEG-based systems, zwitterionic 900 chemistries can be easily incorporated into (subsequently) 901 sensory interfaces via self-assembly or polymerization. The 902 former has been used by Wang et al., who investigated mixed 903 zwitterionic antifouling SAMs of SB and CB-modified thiols on 904 a gold SPR chip for the detection of rabbit polyclonal $\mathrm{IgG}^{158}{ }^{1505}$ The SAM possessed a high fouling resistance whereby the $\mathrm{CB} 906$ also served as an anchor point for antibody immobilization. 907 The sensor exhibited high sensitivity (LoD of $0.4 \mathrm{nM}$ ) and 908 high target specificity without interference from chicken 909 polyclonal IgG. As shown in Figure 15, a CB-based SPR $910 \mathrm{f} 15$

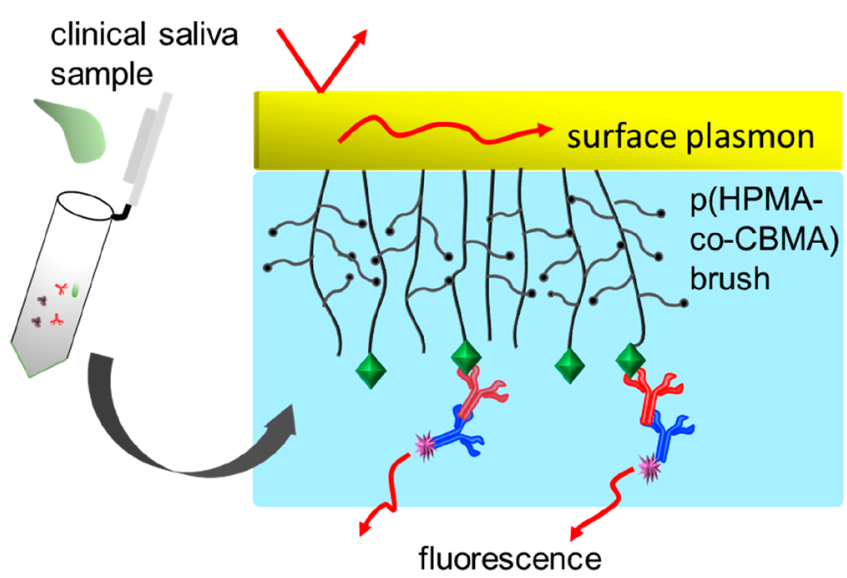

Figure 15. Schematic depiction of a plasmonically enhanced fluorescence biosensor where a gold sensor chip functionalized with a poly(HPMA-co-CBMAA) brush functions as a nonfouling support for Hepatitis B antigen. After anti-Hepatitis B capture, a second fluorophore labeled antibody is introduced enabling sensitive detection anti-Hepatitis B via plasmonically enhanced fluorescence. Adapted with permission from ref 43. Copyright 2017 American Chemical Society.

sensory system for the detection of Hepatitis B antibody in 911 clinical saliva has also been reported by Riedel et al. ${ }^{43}$ In this 912 case, poly[(N-(2-hydroxypropyl)methacrylamide)-co-(carbox- 913 ybetaine methacrylamide)] (poly[HPMA-co-CBMAA]) 914 brushes were synthesized on an initiator-modified Au surface 915 via $\mathrm{ATRP}$ and the Hepatitis B antigen, then immobilized 916 through standard methods. Upon exposure to saliva, the target 917 antibody is bound followed by addition of a fluorophore- 918 labeled secondary antibody ( $\operatorname{IgG}$ ) enabling detection via 919 surface plasmon-enhanced fluorescence. This sandwich 920 immunosensor showed excellent antifouling properties in 921 saliva and was capable of distinguishing between positive 922 clinical saliva samples (respective serum ELISA response $>1$ IU 923 $\mathrm{mL}^{-1}$ ) and negative clinical saliva samples (respective serum 924 ELISA response $<0.01 \mathrm{IU} \mathrm{mL}^{-1}$ ).

A broad range of optical sensors based on zwitterionic 926 antifouling chemistries have also been reported. In one 927 example, pCB functionalized cellulose paper has been applied 928 to detect glucose in undiluted human serum. ${ }^{156}$ To this end, 929 pCB brushes were first grafted from cellulose paper via SI- 930 ATRP, which was incorporated into a microfluidic device. The 931 subsequent colorimetric glucose assay was based on the 932 enzymatic oxidation of iodide to iodine. Compared to bare 933 cellulose paper, the pCB-modified cellulose showed an 934 improved performance (color intensity and response time) 935 

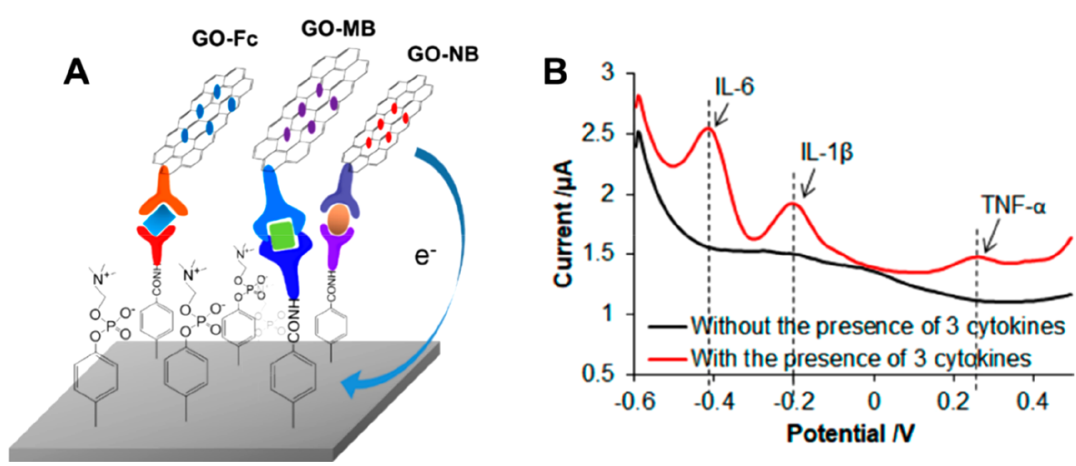

Figure 16. (A) Zwitterionic sandwich immunosensor for the multiplexed detection of three cytokines IL-6, IL-1 $\beta$, and TNF- $\alpha$ in serum. (B) Square wave voltammetry response at the interface before and after incubation with three cytokines IL- 6 , IL- $1 \beta$, and TNF- $\alpha$, followed by incubation of anti-IL-6 Ab-GO-NB, anti-IL-1 $\beta$ Ab-GO-MB, and anti-TNF- $\alpha$ Ab-GO-Fc. Adapted with permission from ref 161 . Copyright 2018 American Chemical Society.

936 for all glucose concentrations $(0.5-5.0 \mathrm{mM})$, particularly in 937 undiluted human serum samples. In other work, optical sensors 938 based on a bifunctionalized silica nanoparticle modified with $939-\mathrm{CB}$ and $-\mathrm{COOH}$ terminated silanes were developed by $\mathrm{Hu}$ 940 et al. ${ }^{113}$ This short-chained interface showed effective 941 antifouling properties in both BSA based model protein 942 solution and 50\% FBS as assessed by the BCA assay. These 943 functionalized nanoparticles were then successfully applied for 944 chemiluminescent detection of human chorionic gonadotro945 phin in 50\% FBS by formation of a sandwich complex on the 946 horseradish peroxidase antibody-modified particles.

947 Brault et al. extended the application of $\mathrm{pCB}$ to the 948 development of an SPR sensor interface capable of the 949 multiplexed profiling of protein targets. ${ }^{159}$ pCB brushes were 950 coated on a gold chip through ATRP and were then 951 functionalized with three antibodies via microcontact printing. 952 Human monoclonal antibody against the activated leukocyte 953 cell adhesion molecule (anti-ALCAM), an antibody against 954 thyroid stimulating hormone (anti-TSH), and an antibody 955 against $\beta$-human chorionic gonadotropin (anti-hCG) were 956 used to generate the printed antibody spots on $\mathrm{pCB}$ films. The 957 authors demonstrated that such a configuration exhibited 958 similar sensitivities to conventional SPR sensors with the same $959 \mathrm{pCB}$ films $\left(10 \mathrm{ng} \mathrm{mL}^{-1}\right.$ vs $\left.7.8 \mathrm{ng} \mathrm{mL}^{-1}\right)$ but with high960 throughput capabilities. This work represents the first 961 demonstration of a low fouling pCB-based antibody sensing 962 array for analysis of undiluted human plasma and shows great 963 potential for the assaying of a wide range of protein analytes. ${ }^{159}$ 964 Similarly, Homola's group has presented a multichannel SPR 965 sensor coated with a functionalizable and low-fouling pCBAA 966 brush for simultaneous detection of E. coli O157:H7 and 967 Salmonella sp. in hamburger and cucumber samples. ${ }^{39}$ The SPR 968 chips were based on a sandwich format of capture $\mathrm{Ab}$-analyte969 streptavidin-labeled detection $\mathrm{Ab}$ on pCBAA brushes. 970 Recognition and binding of biotinylated gold nanoparticles 971 were used to enhance the SPR sensor response and amplify the 972 signal. The limits of detection for the two species of bacteria in 973 cucumber and hamburger extracts were determined to be 57 974 colony-forming unit (CFU) $\mathrm{mL}^{-1}$ and $17 \mathrm{CFU} \mathrm{mL}^{-1}$ for $E$. 975 coli, $7.4 \times 10^{3} \mathrm{CFU} \mathrm{mL}{ }^{-1}$ and $11.7 \times 10^{3} \mathrm{CFU} \mathrm{mL}^{-1}$ for 976 Salmonella sp., respectively.

977 Vaisocherová et al. reported an extraction-free (no pretreat978 ment) and polymerase chain reaction (PCR)-free sensor based 979 on a zwitterionic PCBAA SPR array that enabled the direct 980 multiplexing of four miRNAs (miR-16, miR-181, miR-34a, and $981 \mathrm{miR}-125 \mathrm{~b}$ ) in crude erythrocyte lysates (EL). ${ }^{41}$ pCBAA brushes approximately $40 \mathrm{~nm}$ thick (generated by ATRP) 982 were able to load $\sim 9.8 \times 10^{12}$ probes per $\mathrm{cm}^{2}$ and showed a 983 strong resistance to fouling from EL. samples $\left(<2 \mathrm{ng} \mathrm{cm}^{-2}\right)$. 984 Different capture probes for the four miRNAs were 985 immobilized onto this support facilitating RNA quantification 986 after hybridization with biotinylated detection probes (the 987 signal of the sandwich hybridization assay can be further 988 amplified with the aid of streptavidin-functionalized AuNPs). 989 The developed sensor successfully assayed for miRNA 990 biomarkers of myelodysplastic syndrome in clinical EL samples 991 enabling detection of miRNAs at levels $<0.5 \mathrm{pM}$. 992

Other electrochemical sensors have been explored by Jiang 993 et al., who modified electrodes with short and low-impedance 994 binary aryl layers constructed via the electrodeposition of 995 aryldiazonium salts containing zwitterionic PC or carboxy 996 groups for anchoring biorecognition units. ${ }^{36,56}$ For example, an 997 electrochemical sensor for TNF- $\alpha$ detection has been 998 constructed by in situ simultaneous electrografting of PPC 999 and phenyl butyric acid (PBA) onto indium tin oxide (ITO).$^{54} 1000$ This was employed in an amperometric sandwich assay with a 1001 low LoD $(<1 \mathrm{pM})$ reported for TNF- $\alpha$ in whole blood. A 1002 similar approach has utilized reduced graphene oxide 1003 immobilized onto a gold electrode through diazonium 1004 chemistry. ${ }^{160}$ This interface was then further decorated with 1005 mixed zwitterionic PPC and carboxyphenyl groups via 1006 diazonium reduction onto which anti-TNF- $\alpha$ was coupled. 1007 Subsequent amperometric detection of targets was then carried 1008 out by a sandwich method whereby the recruited analytes were 1009 exposed to ferrocene and anti-TNF- $\alpha$-modified rGO. This 1010 electrochemical immunosensor was successfully used for the 1011 detection of secreted TNF- $\alpha$ from BV-2 cell lines (in cell 1012 media). In a more recent study, the same group reported a 1013 similar sensor capable of multiplexed sensing of cytokines 1014 TNF- $\alpha$, IL-6, and IL-1 $\beta$ in serum. ${ }^{161}$ As shown in Figure 16, $1015 \mathrm{f} 16$ mixed layers of PPC and CP were electrodeposited, followed 1016 by antibody immobilization. Analyte detection was carried out 1017 amperometrically with the rGO both analyte antibody and 1018 redox reporter (nile blue (NB), methylene blue (MB), or 1019 ferrocene $(\mathrm{Fc})$ )-modified. Because of the distinct redox peaks 1020 associated with these reporters $(-0.4 \mathrm{~V},-0.2$ and $0.2 \mathrm{~V}, 1021$ respectively), a simultaneous sensing of all three analytes was 1022 deemed possible in undiluted mouse serum. This study 1023 demonstrates a promising adaptive methodology for multi- 1024 plexed sensing in complex media by rational selection and 1025 evaluation of redox labels. In another study, electrochemical 1026 sensors based on a zwitterionic polymer interface were 1027 


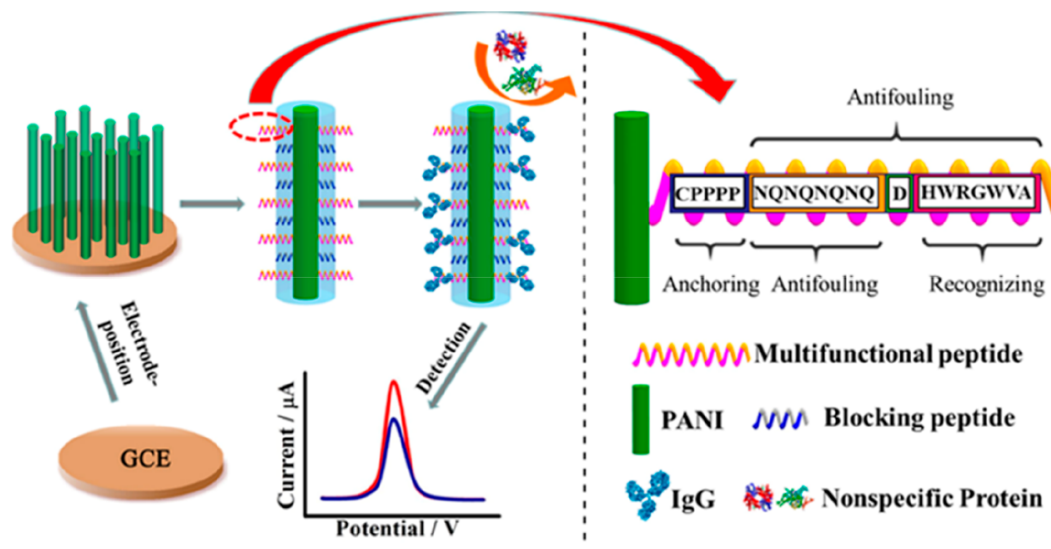

Figure 17. Multifunctional peptide-polyaniline nanowire based sensor for the detection of IgG in serum. Reproduced with permission from ref 164 . Copyright 2018 American Chemical Society.

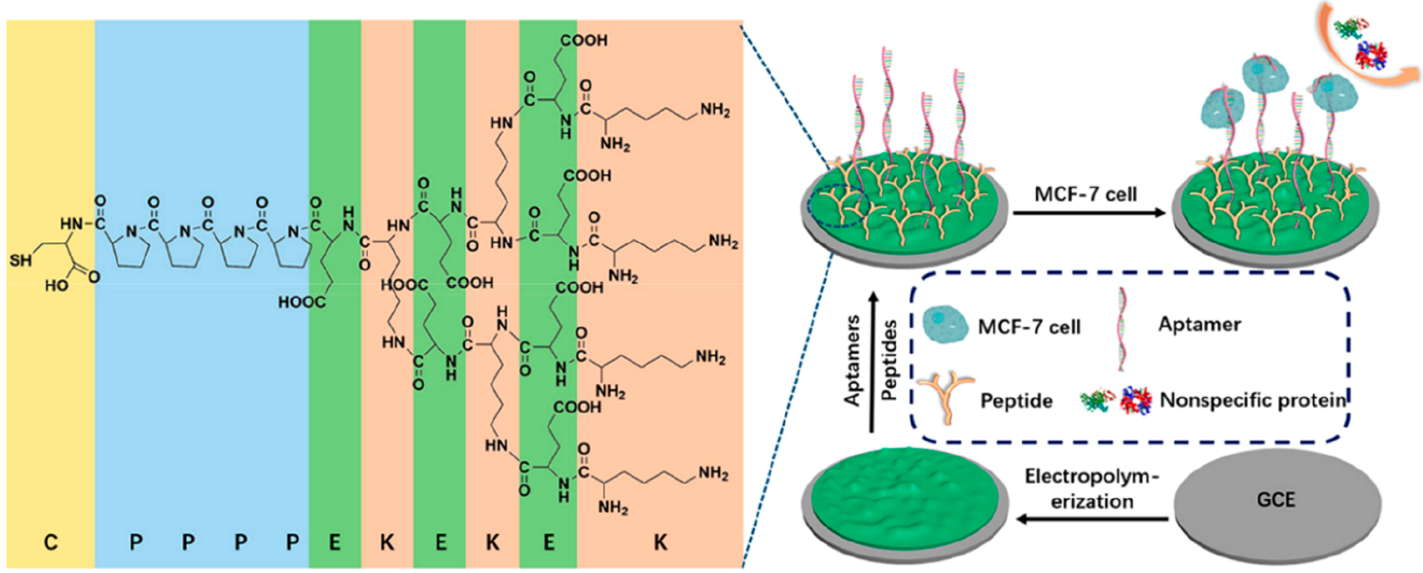

Figure 18. Schematic illustration of the preparation of a PANI-supported branched peptide-based amperometric cell sensor (C, cysteine; P, proline; E, glutamic acid; K, lysine.) Reproduced with permission from ref 168. Copyright 2019 American Chemical Society.

1028 reported by Davis and co-workers who developed a pCBMA 1029 based label-free biosensor to support the $\mathrm{fM}$ detection of 1030 insulin $(\mathrm{LoD}=40 \mathrm{fM})$ in neat serum, which, some years later, 1031 still represents a benchmark in the selective label free 1032 electrochemical detection of protein targets in complex 1033 fluid. ${ }^{102}$ In spite of their frequent use and excellent antifouling 1034 capabilities, few zwitterionic compounds are commercially 1035 available necessitating the often complicated synthesis and 1036 purification of these highly charged molecules. Furthermore, it 1037 should be taken into account that the external $\mathrm{pH}$ as well as 1038 electric field (e.g., in electrochemical sensing) may influence 1039 the overall interfacial charge status and thus the antifouling 1040 behavior.

1041 2.3.2.3. Peptides. Peptides are natively biocompatible with 1042 physicochemical characteristics (hydrophilicity, charge, con1043 formational rigidity, anchor groups) tunable through their 1044 specific sequence. With the high hydrogen bond-donor/ 1045 acceptor abilities of their polar functional groups and their 1046 zwitterionic charge, peptides are usually strongly hy1047 drated. $^{18,162}$ Charged side chains (glutamic acid (E), lysine $1048(\mathrm{~K})$, aspartic acid (D), histidine $(\mathrm{H})$, and arginine $(\mathrm{R}))$ or 1049 surface anchor groups (cysteine $(\mathrm{C})$ ) are usually incorporated 1050 in derived applications, ${ }^{27,34,163}$ with nonfouling character also 1051 being reported on noncharged hydrophilic ${ }^{164}$ and amphiphilic 1052 peptides. ${ }^{165}$ Peptides of various length and sequence have been 1053 assembled onto a range of planar and nanoparticulate surfaces most commonly by incorporation of a cysteine motif at the 1054 peptide terminus to facilitate the formation of ("thiolate") 1055 SAMs on gold. The influence of complex amphiphilic and 1056 zwitterionic peptide structures on the resistance of protein 1057 adsorption has been investigated by $\mathrm{He}$ and co-workers. 1058 Specifically, two different heptapeptides, the noncharged 1059 CYSYSYS and the zwitterionic CRERERE, have been 1060 synthesized and self-assembled on gold. ${ }^{165}$ Ultralow levels of 1061 protein adsorption $\left(1.97-11.78 \mathrm{ng} \mathrm{cm}^{-2}\right.$ ) on these surfaces 1062 were quantified by SPR with single proteins (BSA, lysozyme, 1063 and $\beta$-lactoglobulin) as well as biological media (serum, 1064 soybean milk, and cow milk) confirming an improved 1065 performance of the zwitterionic peptide interface (over the 1066 amphiphilic noncharged peptide) for both single protein 1067 solutions and natural complex fluids.

1068

On the basis of such background observations, a number of 1069 peptide-based sensors have been constructed. In one recent 1070 study, a SPR sensor for the detection of platelet-derived 1071 growth factor (PDGF-BB) was developed. ${ }^{166}$ To this end, a 1072 gold coated optical fiber has been modified with mixed SAMs 1073 of the antifouling CPPPP-EKEKEKE peptide and an aptamer 1074 by simultaneous self-assembly to produce a film that was then 1075 employed in a sandwich format using AuNPs as signal 1076 amplifiers. This enabled PDGF-BB sensing in 10\% human 1077 serum with a sub pM LoD. 


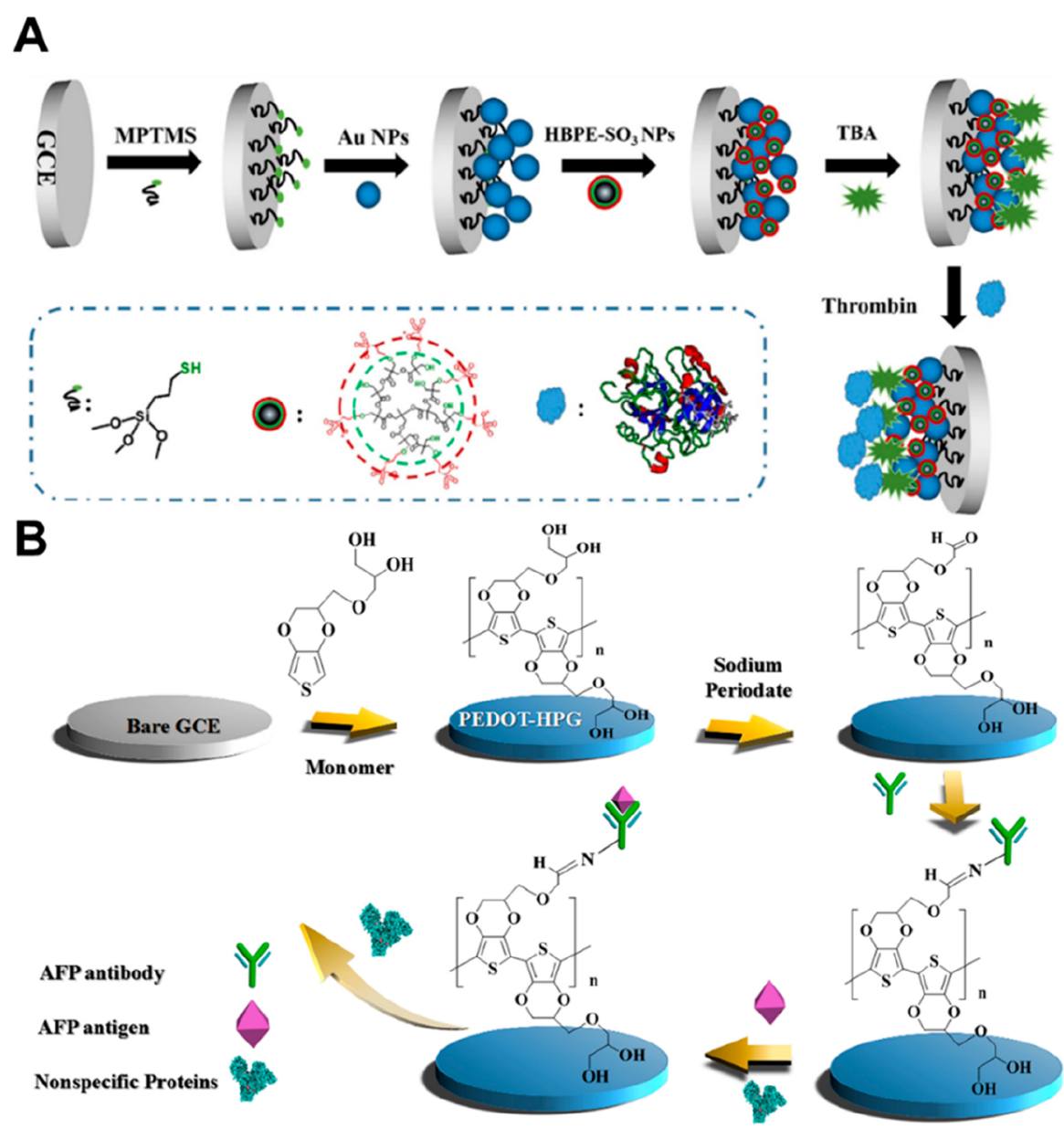

Figure 19. (A) Schematic illustration of the fabrication of a hyperbranched polyester-sulfonic acid nanoparticle-based aptasensor for electrochemical sensing of thrombin in undiluted serum. Reproduced with permission from ref 170. Copyright 2017 Elsevier. (B) Schematic illustration of the fabrication of the AFP biosensor interface using HPG. Reproduced with permission from ref 175 . Copyright 2019 Elsevier.

1079 Peptide films have also been used in a range of electro1080 chemical sensor formats. Luo's group, for example, recently 1081 reported a peptide-based nonfouling interface for the sensitive 1082 detection of IgG in clinical human serum using DPV. ${ }^{164}$ As 1083 illustrated in Figure 17, PANI nanowire arrays were first 1084 constructed on GC electrodes via electropolymerization. 1085 Immobilization of a multifunctional peptide onto this modified 1086 electrode was then carried out via covalent cross-linking of the 1087 amino groups of PANI and thiol groups of the peptide via 1088 sulfo-SMCC. The peptide was specifically designed to contain 1089 an anchoring unit (CPPPP), a nonfouling sequence 1090 (NQNQNQNQ), and a specific IgG recognition sequence 1091 (HWRGWVA). This aptasensor was capable of selective IgG 1092 detection via DPV in neat serum and real clinical samples with 1093 a low pM LoD. The analysis of patient samples was resolved to 1094 be in good agreement with hospital nephelometry IgG 1095 quantifications. The same group employed a EKEKEKE 1096 peptide sequence in assays of BRCA1. ${ }^{33,167}$ Specifically, 1097 PEDOT was electrodeposited onto GC to anchor the 1098 zwitterionic peptide via nickel cation coordination. Receptive 1099 DNA probes were immobilized via EDC/NHS facilitating the 1100 detection of BRCA1 in 5\% human plasma with a sub fM 1101 sensitivity by DPV. ${ }^{145}$ A similar sensing interface on gold has 1102 been utilized for the impedimetric detection of BRCA1 in 10\% 1103 human serum. ${ }^{27}$
In a recent study, a novel branched peptide has been 1104 explored, wherein a mucin 1 aptamer (for capture of MCF-7 1105 cancer cell) and a branched zwitterionic peptide (CPPPPEK- 1106 $\left.(\mathrm{EK})_{2}(\mathrm{EK})_{4}\right)$ were simultaneously immobilized on PANI 1107 coated GC to generate the sensory interface (Figure 18). $1108 \mathrm{f} 18$ The branch-structured peptide was shown to offer better 1109 antifouling performance than that of linear analogues or PEG 1110 (likely related to conformational entropy and higher levels of 1111 hydration). The developed sensor was capable of detecting 1112 cancer cell with a LoD of 20 cells $\mathrm{mL}^{-1}$ in spiked human 1113 serum. $^{168}$

1114

These studies clearly demonstrate the potential of peptide 1115 based antifouling interfaces in sensors whereby their chemical 1116 tunability (i.e., amino acid sequence) can be rationally 1117 designed for a given specific applications. Comparatively little 1118 analysis has been done of the operational mechanisms active at 1119 such, but it is likely that hydration levels exceed those at PEG 1120 or CB/SB/PC-based interfaces. Caution should also be applied 1121 to the use of sequences containing cysteine or methionine 1122 residues where oxidative degradation is possible. Depending on 1123 application, protease degradation may also result in a 1124 compromised antifouling performance (Table 4).

1125

2.3.2.4. Other Antifouling Chemistries. In addition to the 1126 aforementioned chemistries, a myriad of other hydrated 1127 molecular systems have been employed in sensors designed 1128 to recruit targets selectively under testing conditions. For 1129 
1130 example, hyaluronic acid (HA, Figure 1D), an anionic 1131 glycosaminoglycan of polysaccharides, has been explored as 1132 antifouling decoration in several studies. ${ }^{37,169}$ The disaccharide 1133 unit in HA contains amide and carboxylic acid groups are 1134 believed to provide hydrogen-bond donor/acceptor and thus 1135 enhance the hydration that ultimately presents a thermody1136 namic repulsion to proteins. ${ }^{169}$ In the meantime, hyper1137 branched polymers, a class of synthetic dendritic macro1138 molecules with irregularly dense branched structures, and a 1139 large number of functional end groups, are promising 1140 antifouling materials. The surface-immobilization of these 1141 polymers, via, for example, polymerization of glycidol, can 1142 introduce a high density of hydrophilic functional groups on 1143 surfaces facilitating both further functionalization as well as the 1144 formation of a strong hydration layer.

1145 In a recent study, the detection of carcinoembryonic antigen 1146 (CEA) in human serum was demonstrated by construction of a 1147 sensor based on HA and PANI nanowires. ${ }^{31}$ By modification 1148 of HA with conducting PANI, the label-free anti-CEA/HA/ 1149 PANI sensor was capable of sensitive amperometric detection 1150 of CEA with $a>3$ order of magnitude linear response and a fM 1151 detection limit in buffer and a high recovery $(\sim 100 \%)$ in 1152 serum samples.

1153 The use of a heparin-mimicking hyperbranched polyester 1154 nanoparticle based aptasensor for the detection of thrombin in 1155 whole blood has been explored by Niu et al. ${ }^{170}$ As shown in 1156 Figure 19A, the proposed sensor interface was obtained 1157 through a layer-by-layer self-assembly approach, with 31158 mercaptopropyltrimethoxysilane (MPTMS) first grafted onto 1159 the surface of GC to enable Au NPs anchoring. Then 1160 hyperbranched polyester-sulfonic acid nanoparticles (HBPE$1161 \mathrm{SO}_{3} \mathrm{NPs}$ ) were attached via electrostatic adsorption, followed 1162 by immobilization of a $5^{\prime}$-amino thrombin aptamer by 1163 physisorption. This sensing interface showed good resistance 1164 to nonspecific proteins and blood cell adhesion compared to 1165 MPTMS- or HBPE-OH-modified interfaces and was applied 1166 to detect thrombin (TB) in whole blood by DPV with 1167 comparable performance to that of the aptamer-based 1168 fluorescence assay. There are also reports of hyperbranched 1169 polyglycerol (HPG) antifouling coatings on glass, silicon, 1170 porous silicon, and GC electrodes. ${ }^{171-175}$ In one recent 1171 example, HPG-EDOT was electropolymerized onto GC, which 1172 was then partially oxidized by periodate to an antibody 1173 anchoring aldehyde (Figure 19B). ${ }^{175}$ This sensory interface 1174 permitted the sensitive detection of $\alpha$-fetoprotein (AFP) with a $1175 \mathrm{fM}$ LoD in serum by square wave voltammetry (SWV).

1176 Qi et al. have studied a ternary monolayer based TB 1177 aptasensor created by the coassembly of dithiothreitol (DTT), 1178 a thiolated TB aptamer, and 6-mercapto-1-hexanol $(\mathrm{MCH})$ 1179 backfilling on gold. ${ }^{176}$ The hydroxyl groups on DTT and MCH 1180 provide a hydrophilic microenvironment most likely highly 1181 relevant to the observed nonfouling properties. It was 1182 demonstrated that (within an impedimetric assay) the increase 1183 in the electron transfer resistance was linearly proportional to 1184 the concentration of thrombin in the range from 28 to $556 \mathrm{pM}$ 1185 with a detection limit of $8 \mathrm{pM}$. The direct quantification of 1186 extrinsic thrombin in undiluted serum samples was sub1187 sequently achieved.

1188 In other work, McQuistan et al. have explored negatively 1189 charged oligonucleotides as antifouling components together 1190 with a methylene blue (MB) labeled peptide epitope (from 1191 HIV p24 antigen) as probe for a HIV antibody (Figure 20). ${ }^{177}$ 1192 When the target antibody binds to the peptide, the MB probe

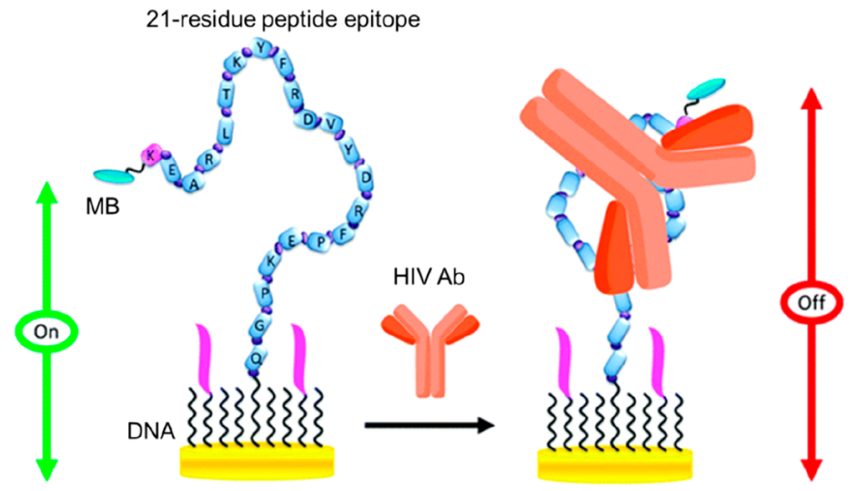

Figure 20. Electrochemical peptide-based sensor with DNA as antifouling diluent for the detection of a HIV antibody in saliva. Before analyte binding the peptide epitope is flexible ("on state") resulting in a measurable methylene blue (MB) tag derived faradaic current. After analyte binding, the associated conformational change results in an attenuated electrochemical response ("off" state). Reproduced with permission from ref 177. Copyright 2014 Royal Society of Chemistry.

flexibility is significantly reduced, leading to a conveniently 1193 resolved reduction in measured current. Different surface ratios 1194 of the mixed layers were investigated revealing an optimal 1195 DNA/peptide surface ratio of $1.37 \times 10^{3}: 1$ in enabling a low 1196 $\mathrm{nM}$ sensitivity assay in saliva. Significantly, the oligonucleotide 1197 was shown to play a crucial role in preventing fouling; 1198 replacement with 6-mercaptohexanol was observed to result in 1199 significant fouling and substantial faradaic signal suppression. 1200 Liús and Meyeŕs groups have recently expanded the scope of 1201 DNA-based antifouling interfaces by immobilizing DNA 1202 triangle nanostructures on $\mathrm{SiO}_{2}$ through DNA-lithography 1203 and formation of layer-by-layer assemblies with polycations 1204 (i.e., poly(ethyleneimine), PEI) on glass, respectively. ${ }^{178,179} 1205$ Both interfaces have been observed to effectively resist biofilm 1206 formation. In related work, DNA tetrahedra on gold have been 1207 shown to exhibit excellent antifouling performance ( $\leq 8.0 \mathrm{ng} 1208$ $\mathrm{cm}^{-2}$ ) even in neat serum. ${ }^{180}$ These and related configurations 1209 are likely to be of value in further extending DNA based 1210 interfaces for highly selective biosensing in complex biofluids. 1211

In contrast to the aforementioned negatively charged DNA- 1212 based antifouling interfaces, a recent report investigated the 1213 performance of the positively charged lipid bilayer mimic, 2- 1214 dioleoyl-sn-glycero-3-ethylphosphocholine $\left(\mathrm{EPC}^{+}\right)$, as a non- 1215 fouling support. ${ }^{181}$ As shown in Figure 21A, a gold chip was $1216 \mathrm{f} 21$ first modified with a mixed SAM of 3-mercapto-1-propanol 1217 (MPO) and protein A. The latter was utilized for the 1218 subsequent oriented recruitment of anti-IgG after which a 1219 nonfouling lipid membrane was introduced. Interestingly, the 1220 natural, zwitterionic, 1-palmitoyl-2-oleoyl-sn-glycero-3-phos- 1221 phocholine (POPC) membrane displayed higher fouling than 1222 the positively charged EPC membrane, which was sub- 1223 sequently utilized to support the detection of IgG in undiluted 1224 mouse serum in a sandwich format via SPR (Figure 21B). 1225 Special attention should be drawn to the necessary precise 1226 control over the lipid bilayer formation and antibody 1227 integration into this architecture. It should also be noted that 1228 in this, and previous examples of permanently charged 1229 interfaces, a clear rational for the observed antifouling 1230 performance is lacking; while these interfaces are certainly 1231 strongly hydrated, biofouling from oppositely charged (bio)- 1232 molecules could be expected. These concerns thus require the 1233 

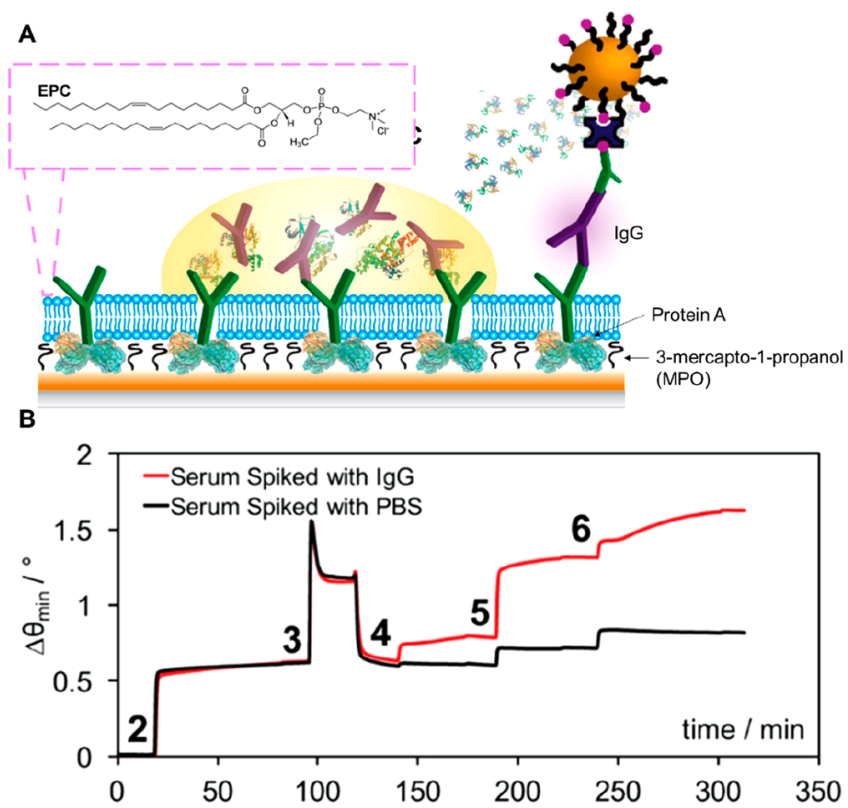

Figure 21. (A) Schematic representation of a sensory interface for IgG detection in undiluted serum based on a positively charged lipid bilayer coating. (B) SPR sensogram showing the assembly of the interface and subsequent IgG detection. After self-assembly of the underlying $\mathrm{MPO} /$ protein $\mathrm{A} /$ anti-IgG interface, EPC lipid vesicles were utilized to form the lipid bilayer (2), after which serum spiked with mouse IgG was injected (3), followed by a biotinylated detection antibody (4), a streptavidin bridge (5), and biotin-labeled gold nanoparticles (6) for signal enhancement. Adapted with permission from ref 181. Copyright 2019 American Chemical Society.

1234 further theoretical and experimental investigation. It is worth 1235 noting that a similar strategy based on biomimetic cell 1236 membranes, for example, human platelet membrane-coated 1237 poly(lactic-co-glycolic acid) (PLGA) particles $^{182}$ or red blood 1238 cell membrane-coated gold nanoparticles, ${ }^{183}$ has been applied 1239 to drug delivery and therapeutics, where the membrane coating 1240 apparently confers the nanoparticles with platelet-mimicking 1241 properties reducing cellular uptake by macrophage-like cells 1242 and securing a prolonged circulation time.

1243 An interesting addition to the chemical antifouling approach 1244 was recently reported by Demirci et al. ${ }^{120}$ The authors 1245 reported a stimuli-responsive sensing interface that can switch 1246 between two states: an antifouling/repelling state and a 1247 detection state. This was achieved by the formation of a 1248 mixed polymeric film on silicon whereby long polymer brushes 1249 of poly(2-N-morpholinoethyl methacrylate) (PMEMA) serve 1250 as the antifouling decoration, while a shorter beta-cyclodextrin $1251(\beta$-CD)-terminated polymer is utilized as an anchor to 1252 immobilize an adamantylamine-modified capture antibody. In 1253 this "resting" state, the longer, extended PMEMA chains 1254 completely cover the $\beta$-CD anchor groups preventing 1255 passivating the surface. At temperatures above $37{ }^{\circ} \mathrm{C}$, the 1256 PMEMA brush collapses and exposes the $\beta$-CD. This interface 1257 can then be modified with the capture antibody and utilized to 1258 detect the target analyte in a fluorescent sandwich format. As a 1259 proof-of-concept, the authors demonstrate that this state can 1260 indeed be utilized to qualitatively detect antihepatitis C in PBS. 1261 This platform can potentially be adapted to other antifouling/ 1262 receptor functionalities to create switchable sensing formats.

1263 To summarize so far, a range of antifouling chemistries have 1264 been applied in a multitude of electrochemical and optical sensors that are capable of detecting different biomarkers in 1265 complex samples and more examples based on the design 1266 strategy (illustrated in Figure 9) are summarized in Table 2, $1267 \mathrm{t} 2$ whereby different chemical configurations present different 1268 advantages and limitations. To overcome the functional 1269 chemical or performance limitations that are frequently 1270 associated with the use of a single chemical entity, recent 1271 work has progressively moved toward the use of combinations. 1272 To date, these have included, for example, the use of mixed 1273 assemblies based on a combination of, for example, PEG/ 1274 $\mathrm{HA},{ }^{184} \mathrm{PC} / \mathrm{HA},{ }^{185} \mathrm{GSH} / \mathrm{HA},{ }^{186}$ or $\mathrm{PDA} / \mathrm{HA},{ }^{187,188}$ peptide/ 1275 $\mathrm{HA}^{189}$ or HA, PC, PDA with lubricin. ${ }^{190-192}$ Currently, 1276 zwitterionic (including peptidic) and polymeric interfaces 1277 currently represent the most tunable high performance 1278 platforms encompassing not only significant antifouling 1279 properties but also structural adaptability, and it is likely that 1280 a continued exploration will furnish a realistic translation to in 1281 vitro PoC diagnostics. Interestingly, some of the above 1282 interfacial designs have also been adapted for the in vivo 1283 monitoring of a range of analytes, as discussed in the next 1284 section.

2.3.3. In Vivo Sensing Based on Antifouling Chem- 1286 istries. In vivo sensors are designed to work in the internal 1287 microenvironment of the body to sense specific environmental 1288 change relevant to health management or an improved 1289 understanding of physiological processes. ${ }^{210-212}$ In compar- 1290 ison to in vitro sensors, in vivo sensors encounter harsher, and 1291 more prolonged, challenges associated with biofouling (e.g., 1292 proteins and cells) as well as potential immune reactions in 1293 response to the introduction of the sensor into the active body. 1294 These challenges brought about by the complex and dynamic 1295 microenvironment can partially or completely impede any pre- 1296 engineered sensing capability in terms of accuracy and usable 1297 lifetime. ${ }^{213,214}$ Antifouling approaches have to be biocompat- 1298 ible with low toxicity and also highly tolerant of the in vivo 1299 dynamic environment, which includes exposure to dissolved 1300 oxygen, transition metals, and enzymes. Thus, the need for 1301 high performance nonfouling designs in such work is crucial; to 1302 date, functional sensors consist of two main phenotypes: 1303 implanted electrochemical sensors and circulating (nano- 1304 particulate) optical sensors. The former are generally capable 1305 of high analytical sensitivity and high temporal resolution and 1306 are most commonly based on cylindrically shaped wire or 1307 multielectrode needle-based microelectrodes (size of a few tens 1308 of micrometers or less), which are directly inserted into 1309 tissue. $^{214,215}$ As with all sensors, biofouling on the sensory 1310 interface can result in profound problems associated with loss 1311 of analyte mass transport or (electric) communication between 1312 the (implantable) in vivo sensor and the body. ${ }^{216,217}$ A broad 1313 range of nanoparticulate platforms such as semiconducting 1314 quantum dots (QDs), metallic nanoparticles, mesoporous 1315 particles, and metal oxide particles have been designed for in 1316 vivo applications like drug delivery, sensing, or imaging. The 1317 decoration of such solid supports with antifouling chemistry is 1318 key to minimizing the formation of an associated protein 1319 corona and can be an absolute requirement if both colloidal 1320 stability and designed functionality are to be retained in 1321 vivo. ${ }^{218,219}$ For example, PEGylated iron oxide nanoparticles 1322 (biotin-PEG, $5 \mathrm{kDa}$ ) and zwitterionic dopamine sulfonate 1323 coated iron oxide nanoparticles have been shown to possess 1324 low levels of toxicity and to prevent phagocytic uptake in 1325 vivo. ${ }^{20,221}$ Similarly, CdSe(ZnCdS) core(shell) QDs with a 1326 compact cysteine coating $\left(D_{\mathrm{h}}<6 \mathrm{~nm}\right)$ were found to prevent 1327 
Table 2. Fabrication of Sensors Using Different Antifouling Molecular Systems and Their In Vitro Sensing Performance ${ }^{a}$

\begin{tabular}{|c|c|c|c|c|c|c|c|}
\hline & sensor & & & sensing perfo & nance & & \\
\hline $\begin{array}{l}\text { antifouling } \\
\text { unit }\end{array}$ & overall composition & analyte & $\begin{array}{l}\text { detection } \\
\text { method }\end{array}$ & dynamic range & detection limit & complex sample & ref \\
\hline OEG & $\begin{array}{l}\text { Fc-GO-detection Ab/troponin-I/Ab1/GO- } \\
\text { Ph-AuNPs-Ph-OEG/Ph-amine/GC }\end{array}$ & troponin-I & SWV & $2.1-126 \mathrm{pM}$ & $2.1 \mathrm{pM}$ & human plasma & 193 \\
\hline PEG & $\begin{array}{l}\text { Anti-insulin Ab/PEGylated thiol-backfilled } \\
\text { BSA/Au }\end{array}$ & insulin & EIS & $5 \mathrm{pM}-50 \mathrm{nM}$ & $1.2 \mathrm{pM}$ & human serum & 123 \\
\hline PEG & Anti-CRP Ab/PEG thiol-Fc/Au & CRP & EIS & $0.5 \mathrm{pM}-50 \mathrm{nM}$ & $1 \mathrm{pM}$ & human serum & 125 \\
\hline PEG & Anti-CRP Ab/PEG thiol/Au & CRP & EIS & $0.5-50 \mathrm{nM}$ & $176 \mathrm{pM}$ & human serum & 126 \\
\hline PEG & $\mathrm{Ab} / \mathrm{PEG} / \mathrm{AuNPs} / \mathrm{PANI} / \mathrm{GC}$ & AFP & DPV & $147 \mathrm{aM}-14.7 \mathrm{pM}$ & $103 \mathrm{aM}$ & human serum & 115 \\
\hline PEG & Capture DNA/PEG/PANI/GC & BRCA1 & DPV & $0.01 \mathrm{pM}-1 \mathrm{nM}$ & $3.8 \mathrm{fM}$ & human serum & 38 \\
\hline PEG & Aptamer-PEG/PDA/GC & ATP & EIS & $0.1-1000 \mathrm{pM}$ & $0.1 \mathrm{pM}$ & $\begin{array}{l}\text { human plasma; cancer } \\
\text { cell lysates }\end{array}$ & 194 \\
\hline PEG & $\mathrm{Ab} / \mathrm{PEG}$ epoxide-PEG amine/Au & insulin, CRP & EIS & $0.5-100 \mathrm{pM}, 0.5-50 \mathrm{nM}$ & $171 \mathrm{fM}, 150 \mathrm{pM}$ & human serum & 131 \\
\hline PEG & $\mathrm{Abl} / \mathrm{SH}-\mathrm{PEG}-\mathrm{COOH} / \mathrm{Au}$ & $\alpha$-synuclein & EIS & $0.5-10 \mathrm{nM}$ & $55 \mathrm{pM}$ & human serum & 47 \\
\hline PEG & $\mathrm{Ab} /$ thiolated $\mathrm{PEG}$ acid/Au & IL-8 & EIS & $81 \mathrm{fM}-81 \mathrm{nM}$ & $8.1 \mathrm{fM}$ & human serum & 195 \\
\hline PEG & $\begin{array}{l}\text { Brucellosis } \mathrm{Ab} / \mathrm{OMP} 31 / \mathrm{Fe}_{3} \mathrm{O}_{4} / \mathrm{Au} / \mathrm{PEG} / \\
\text { HA NPs/GC }\end{array}$ & Brucellosis Ab & DPV & $50 \mathrm{aM}-500 \mathrm{fM}$ & $18 \mathrm{aM}$ & human serum & 196 \\
\hline EGDMA & $\begin{array}{l}\text { TB aptamer/p(HEMA-EGDMA-VC) } \\
\text { /microbeads/gold chip }\end{array}$ & thrombin & QCM & $1-100 \mathrm{nM}$ & $1 \mathrm{nM}$ & human serum & 197 \\
\hline PEG & $\begin{array}{l}\text { reporter-antiEpCAM/EVs/anti-EpCAM/ } \\
\text { PEG/Pt }\end{array}$ & tdEVs & $\mathrm{CV}$ & $10-10^{6}$ tdEVs $\mu \mathrm{L}^{-1}$ & 5 tdEVs $\mu \mathrm{L}^{-1}$ & cell media & 198 \\
\hline PPC & HRP-Ab2/analyte/Ab1/PPC-PBA/ITO & TNF- $\alpha$ & CA & $0.57 \mathrm{pM}-28.5 \mathrm{nM}$ & $0.57 \mathrm{pM}$ & human blood & 54 \\
\hline PPC & $\begin{array}{l}\text { GO-Ab2-redox probes }(\mathrm{NB} / \mathrm{Fc} / \mathrm{MB}) / \\
\text { analyte/Ab1/PPC-CP/GC }\end{array}$ & $\begin{array}{l}\text { IL-6, IL- } 1 \beta \text {, } \\
\text { TNF- } \alpha\end{array}$ & SWV & $\begin{array}{l}0.21-6.2 \mathrm{pM} \\
0.16-6.4 \mathrm{pM} \\
0.285-11.4 \mathrm{pM}\end{array}$ & $\begin{array}{l}0.21 \mathrm{pM}, 0.16 \\
\mathrm{pM}, 0.285 \mathrm{pM}\end{array}$ & mouse serum & 161 \\
\hline PPC & $\begin{array}{l}\text { Ab2-GO-Ph-Fc/TNF- } \alpha / \mathrm{Ab} 1 / \mathrm{PPC}-\mathrm{CP} / \\
\text { rGO-AuNPs/Ph/Au }\end{array}$ & TNF- $\alpha$ & SWV & $5.7 \mathrm{fM}-8.55 \mathrm{pM}$ & $5.7 \mathrm{fM}$ & $\begin{array}{l}\text { cell conditioned } \\
\text { media }\end{array}$ & 160 \\
\hline pCBMA & pCBMA/cysteamine/Au & insulin & EIS & $0.1-200 \mathrm{pM}$ & $42.6 \mathrm{fM}$ & human serum & 102 \\
\hline PVIS & PVIS-GMA-Ab/GO/GC & streptomycin & DPV & $0.09-171 \mathrm{nM}$ & $3 \mathrm{pM}$ & milk & 199 \\
\hline peptide & $\mathrm{MCH} /$ Aptamer-Peptide/AuNPs/Au & ATP & EIS & $0.1 \mathrm{pM}-5 \mathrm{nM}$ & $0.1 \mathrm{pM}$ & $\begin{array}{l}\text { human whole blood; } \\
\text { cancer cell lysates }\end{array}$ & 105 \\
\hline peptide & ssDNA/Peptide $/ \mathrm{Ni}^{2+} /$ Citrate-PEDOT/GC & BRCA1 & DPV & $10 \mathrm{fM}-10 \mathrm{nM}$ & $0.03 \mathrm{fM}$ & human plasma & 167 \\
\hline peptide & $\begin{array}{l}\text { mixed antifouling peptide-blocking peptide/ } \\
\text { PANI/GC }\end{array}$ & IgG & DPV & $6.7 \mathrm{pM}-67 \mathrm{nM}$ & $1.7 \mathrm{pM}$ & human serum & 164 \\
\hline peptide & mixed antifouling peptide-AFP aptamer/Au & AFP & DPV & $147 \mathrm{aM}-1.47 \mathrm{pM}$ & $45.6 \mathrm{aM}$ & human serum & 200 \\
\hline peptide & $\begin{array}{l}\text { MTase/Au@luminol-H1/peptide/AuNPs/ } \\
\text { PANI/ITO }\end{array}$ & MTase & ECL & $0.05-100 \mathrm{U} \mathrm{mL}^{-1}$ & $0.02 \mathrm{U} \mathrm{mL}^{-1}$ & human serum & 201 \\
\hline peptide & $\begin{array}{l}\text { Ig E aptamer/zwitterionic peptide/porous } \\
\text { gold/GC }\end{array}$ & $\operatorname{Ig} \mathrm{E}$ & DPV & $5.1 \mathrm{fM}-0.51 \mathrm{pM}$ & $2.1 \mathrm{fM}$ & FBS & 202 \\
\hline peptide & $\begin{array}{l}\text { aptamer/MCH/Peptide/ } \mathrm{ZnIn}_{2} \mathrm{~S}_{4} / \mathrm{TiO} 2 / \\
\text { ITO }\end{array}$ & Hela cell & PEC & $100-10^{6}$ cells $\mathrm{mL}^{-1}$ & 34 cell $\mathrm{mL}^{-1}$ & cell media & 203 \\
\hline peptide & $\begin{array}{l}\text { AuNPs@Fe-MOF-detection DNA/target } \\
\text { DNA/peptide/capture DNA/Au }\end{array}$ & T4 PNK & DPV & $1.0 \times 10^{-3}-10 \mathrm{U} \mathrm{mL}^{-1}$ & $\begin{array}{l}3.5 \times 10^{-4} \\
\mathrm{U} \mathrm{mL}\end{array}$ & human serum & 204 \\
\hline $\begin{array}{l}\text { zwitterion- } \\
\text { peptide }\end{array}$ & sulfophenyl-aminophenyl-S7 peptide/GC & $\begin{array}{r}\text { Streptococcus } \\
\text { pneumonia }\end{array}$ & EIS & $50-5 \times 10^{4} \mathrm{CFU} \mathrm{mL} \mathrm{m}^{-1}$ & $50 \mathrm{CFU} \mathrm{mL} \mathrm{m}^{-1}$ & human serum & 205 \\
\hline HA & $\mathrm{CEA} /$ anti-CEA Ab/HA/PANI/GC & CEA & DPV & $0.56 \mathrm{fM}-0.56 \mathrm{nM}$ & $0.42 \mathrm{fM}$ & human serum & 37 \\
\hline PEG-HA & CD44/PEG-HA-PDA/ $\mathrm{TiO}_{2} / \mathrm{ITO}$ & $\mathrm{CD} 44$ & PEC & $0.06 \mathrm{pM}-6.25 \mathrm{nM}$ & $5.5 \mathrm{fM}$ & human serum & 184 \\
\hline $\mathrm{MCH}$ & aptamer-MCH/PDA/AgNPs/GC & TB & EIS & $0.1 \mathrm{pM}-5.0 \mathrm{nM}$ & $36 \mathrm{fM}$ & human serum & 206 \\
\hline $\begin{array}{l}\text { backfilled } \\
\text { MEA }\end{array}$ & Ab-MEA/PEDOT-HA/GC & CEA & DPV & $56 \mathrm{fM}-5.6 \mathrm{nM}$ & $16.8 \mathrm{fM}$ & human serum & 207 \\
\hline DTT, MCH & aptamer-DTT-MCH/Au & TB & EIS & $27.8-556 \mathrm{pM}$ & $8.3 \mathrm{pM}$ & human serum & 176 \\
\hline DTT, MCH & $\begin{array}{l}\text { HRP-aptamer@AuNPs/lysozyme/MCH/ } \\
\text { DTT/aptamer/Au }\end{array}$ & lysozyme & DPV & $0.71 \mathrm{pM}-7.1 \mathrm{nM}$ & $0.2 \mathrm{fM}$ & human serum & 208 \\
\hline polyglycerol & antiAFP $\mathrm{Ab} / \mathrm{PEDOT}-\mathrm{HPG} / \mathrm{GC}$ & AFP & SWV & $1.4 \mathrm{fM}-14 \mathrm{pM}$ & $0.49 \mathrm{fM}$ & human serum & 175 \\
\hline PA-PANI & antiCRP $\mathrm{Ab} / \mathrm{PA}-\mathrm{PANI} / \mathrm{SPGE}$ & CRP & EIS & $2.25 \mathrm{nM}-144 \mathrm{nM}$ & $4.5 \mathrm{nM}$ & FBS & 209 \\
\hline
\end{tabular}

${ }^{a}$ Abbreviations: Fc, ferrocene; GO, graphene oxide; ph, phenyl; CRP, C-reactive protein; EIS, electrochemical impedance spectroscopy; PANI, polyaniline; HA, hyaluronic acid; p(HEMA-EGDMA-VC), poly(2-hydroxyethyl methacrylate-ethylene glycol dimethacrylate-vinylene carbonate); DPV, differential pulse voltammetry; QCM, quartz crystal microbalance; CA, chronoamperometry; CV, cyclic voltammetry; tdEVs, tumor derived extracellular vesicles; SWV, square wave voltammetry; Ag, antigen; Ab1, capture antibody; Ab2, detection antibody; NB, nile blue; MB, methylene blue; ITO, indium tin oxide; AFP, alpha-fetoprotein; PEDOT, poly(3,4-ethylenedioxythiophene); MCH, 6-mercapto-1-hexanol; DTT, dithiothreitol; MEA, monoethanolamine; CEA, carcinoembryonic antigen; IL-6, Interleukin-6; IL-1 $\beta$, Interleukin-1 $\beta$; CP, carboxylic phenyl; $\mathrm{MPH}$, methylparathionhydrolase; Ig E, immunoglobulin E; PVIS, 1-propyl-3-vinylimidazole sulfonate; GMA, glycidyl methacrylate; ECL, electrogenerated chemiluminescence; DNA MTase, DNA methyltransferase; TB, thrombin; PEC, photoelectrochemistry; AgNPs, silver nanoparticles; T4 PNK, T4 polynucleotide kinase; CFU, colony-forming unit; MOF, metal-organic frameworks; HPG, hyperbranched polyglycerol; CRP, C-reactive protein; SPGE, screen-printed graphene nanoplate electrode; PA-PANI, phytic acid-doped polyaniline; FBS, fetal bovine serum. 


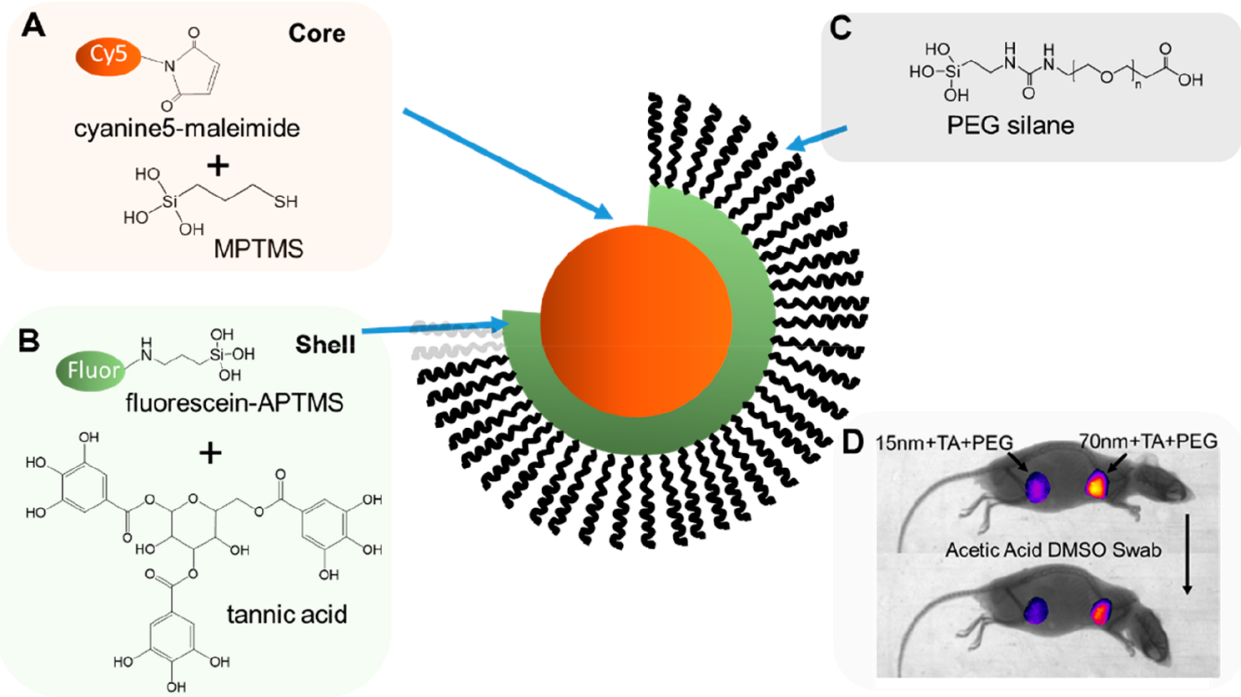

Figure 22. Schematic representation of the synthesis and application of a PEG-coated organosilica core/shell nanoparticle based optical pH sensor. Adapted with permission from ref 233. Copyright 2018 American Chemical Society.

1328 nonspecific serum protein adsorption (as assessed by 1329 DLS). ${ }^{222,223}$ Breus et al. have further investigated coating $1330 \mathrm{CdSe}-\mathrm{ZnS}$ core-shell QDs with zwitterionic D-penicillamine 1331 and demonstrated an antifouling performance, which was 1332 comparable to cysteine coated QDs. ${ }^{24}$ Such antifouling in vivo 1333 nanovectors pave a way for the development of in vivo 1334 nanosensors when further modification with receptive and 1335 transductive elements is carried out. The most successful 1336 examples of such sensors based on the two phenotypes will be 1337 discussed in the following sections.

1338 2.3.3.1. PEG. Benefiting from a commercial availability, high 1339 biocompatibility, and low toxicity, PEG has been the most 1340 frequently used antifouling agent for in vivo applications. For 1341 example, Kozai et al. have reported an ultrasmall implantable 1342 composite microelectrode that enabled single-neuron record1343 ing in rats. ${ }^{225}$ The integrated composite electrode $(\sim 8.5 \mu \mathrm{m}$ 1344 diameter) consists of a carbon fiber core $(7 \mu \mathrm{m}$ diameter $)$ 1345 coated with $50 \mathrm{~nm}$ poly ( $p$-xylylene) as well as a $200 \mathrm{~nm}$ thick 1346 PEG methacrylate polymer as an antifouling layer (both 1347 generated by ATRP). The microelectrodes were implanted 1.6 $1348 \mathrm{~mm}$ into the rat motor cortex to facilitate electrophysiological 1349 recordings. They were reported to provide a stable neural 1350 signal over 5 weeks in the brain without signal degradation, an 1351 important step toward the realization of long-lasting im1352 plants. $^{225}$ On this note, Schwerdt et al. have reported another 1353 ultrasmall sensor capable, apparently, of tracking and 1354 monitoring dopamine in the rat brain for up to one year. ${ }^{226}$ 1355 For this purpose, the authors used an electrode with a diameter 1356 as small as individual neuronal cell bodies $(10 \mu \mathrm{m})$. One 1357 advantage of using electrodes of this scale is that they do not 1358 produce assay-interfering scar tissue. In the study, a thin layer $1359(0.7-1.3 \mu \mathrm{m})$ of parylene-C (a Food and Drug Administration 1360 (FDA)-approved biocompatible dielectric) was deposited on a 1361 bare carbon fiber, and $\mathrm{PEG}_{6000}(0.5-1 \mathrm{~mm}$ thick) was then 1362 cast onto the electrode (by melting) to facilitate implantation 1363 into the rat brain. The microelectrode was exposed at the tip 1364 and then implanted into the forebrain bundle to monitor the 1365 release of DA by fast-scan cyclic voltammetry. It was 1366 demonstrated that, with the incorporation of the PEG coating, 1367 low noise and high sensitivity amperometric performance 1368 could be retained enabling a longitudinal tracking of dopamine over chronic time scales without signal degradation. These 1369 sensors could be useful in Parkinson's patients on receipt of 1370 deep brain stimulation (with additionally implanted stimula- 1371 tion electrodes). Using a sensor to monitor dopamine levels 1372 could help doctors deliver the stimulation more selectively, 1373 only when it is needed. ${ }^{227}$ Inspiringly, existing commercial 1374 systems like closed loop deep brain stimulation systems with 1375 sensing electrodes have been tested in clinical research to 1376 provide continuous neural signal monitoring and therapy for 1377 patients with chronic neuropathic pain. ${ }^{228-230} 1378$

In addition to sensors capable of monitoring neuro- 1379 transmitters, a range of sensor electrodes have been generated 1380 to enable ion level analysis in vivo. Machado et al., for example, 1381 studied an impedimetric sensor for monitoring extracellular 1382 potassium in the mouse brain. ${ }^{231}$ Imbalances in $\left[\mathrm{Na}^{+}\right]$and 1383 $\left[\mathrm{K}^{+}\right]$have been implicated in numerous central nervous system 1384 (CNS) disorders such as epilepsy. ${ }^{232}$ In their study, mixed 1385 monolayers of 18-crown-6-ether (as the $\mathrm{K}^{+}$probe) and 1386 thiolated monoethylene glycol with a molar ratio of 1:10 1387 were formed on gold microelectrode arrays allowing real-time 1388 in vivo non-Faradaic impedimetric measurements of extrac- 1389 ellular $\left[\mathrm{K}^{+}\right]$in the mouse brain (although only over multiple 1390 minutes).

1391

Robinson et al. proposed an organosilica core-shell 1392 ratiometric nanosensor capable of the continuous monitoring 1393 of $\mathrm{pH}$ in the skin of mice via in vivo fluorescence imaging 1394 following subcutaneous injection (Figure 22). ${ }^{233}$ Both core $1395 \mathrm{f} 22$ and shell were formed via a silane based hydrolysis- 1396 condensation procedure. $^{234}$ The $\mathrm{pH}$-sensitive fluorophores 1397 Cy5-maleimide or fluorescein-(3-aminopropyl)- 1398 trimethoxysilane (APTMS) were mixed into the hydrolyzed 1399 (3-mercaptopropyl)trimethoxysilane (MPTMS) solution to 1400 allow covalent incorporation into the core and shell structure, 1401 respectively. A $1 \mathrm{kDa}$ PEG antifouling layer was then 1402 introduced through silanization on the shell. This ratiometric 1403 fluorescence sensor was highly sensitive to $\mathrm{pH}$ over a 1404 physiologically relevant range $(\mathrm{pH} 4.5-8.0)$ with a fast 1405 response time of $<100 \mathrm{~ms}$. The nanosensors were shown to 1406 successfully monitor $\mathrm{pH}$ changes within a bacterial culture over 1407 $15 \mathrm{~h}$. In vivo $\mathrm{pH}$ sensing was then examined by monitoring the 1408 fluorescence after injection of the nanosensors into the 1409 

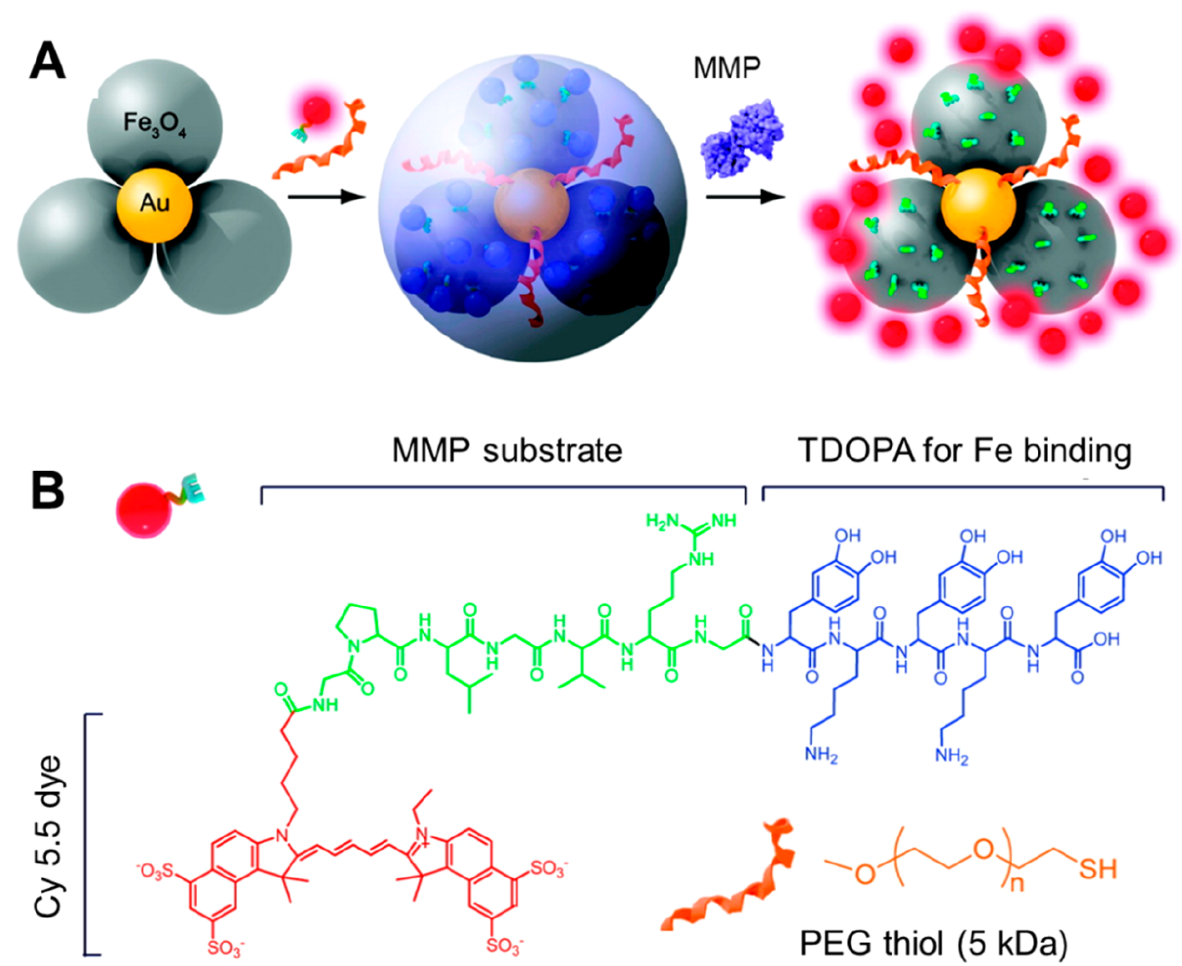

Figure 23. (A) Schematic illustration of the formation of an $\mathrm{Au}-\mathrm{Fe}_{3} \mathrm{O}_{4}$ nanocomposite matrix metalloproteinase (MMP) optical sensor comprising Cy5.5-GPLGVRG-TDOPA and SH-PEG 5000 . (B) PEG integration into the Au-core endows the particles with fouling resistance while the MMP substrate is tagged with TDOPA for assembly onto the $\mathrm{Fe}_{3} \mathrm{O}_{4}$ domains. The substrate is furthermore conjugated to a Cy5.5 dye, which, upon cleavage by MMP, is liberated and strongly fluoresces (initially the fluorescence is quenched on the particle through the underlying gold). Adapted with permission from ref 240. Copyright 2011 American Chemical Society.

1410 subcutaneous tissue of mouse flank skin. After injection, the 1411 skin surface was swabbed with an acidifying solution prior to 1412 imaging at defined times thereafter (to ensure sufficient time 1413 for sample diffusion). The fluorescein and Cy5 emissions were 1414 isolated using spectral unmixing and overlaid with X-ray 1415 images, showing a significant and localized $\mathrm{pH}$ response 1416 (Figure 22D). Notably, the PEG coating resulted in higher 1417 colloidal stability of the optical nanosensor and a better signal1418 to-noise ratio than for the unmodified control nanoparticles or 1419 those nanoparticles containing either tannic acid or PEG alone. 1420 In another use of PEG-based in vivo systems, Clark's group 1421 studied a phosphorescent nanosensor for the monitoring of 1422 histamine. ${ }^{235}$ The $\sim 100 \mathrm{~nm}$ sensor particles were fabricated by 1423 the encapsulation of a sodium optode, diamine oxidase, $\mathrm{Pt}(\mathrm{II})$ 1424 meso-tetra(pentafluorophenyl)porphine (PtTPFPP), and a 1425 hydrophobic platinum porphyrin dye into amphiphilic PEG 1426 lipids via an established nanoemulsion approach. ${ }^{236}$ In this 1427 design, PtTPFPP acts as an oxygen responsive element by 1428 exploiting a reversible, oxygen-dependent phosphorescence 1429 signal. In the presence of oxygen and histamine, diamine 1430 oxidase enzymatically produces ammonia and imidazole-41431 acetaldehyde, which lowers the local oxygen concentration 1432 resulting in increased phosphorescence. A surface coating with 1433 a PEG-lipid (12 EG units) was shown to minimize protein 1434 fouling and prolong nanoparticle lifetime (circulation) as 1435 revealed by liver or kidney tests. In vivo monitoring of 1436 phosphorescence every $30 \mathrm{~s}$ demonstrated that the injected 1437 nanosensor facilitated a continuous monitoring of histamine in 1438 live mice across several hours. This study highlights the 1439 potential of such approaches to widen the range of measurable 1440 analytes by incorporating an enzymatic recognition element; the modular approach applied to histamine sensing is readily 1441 extendable to lactate, creatinine, urea, etc. simply by swapping 1442 out the enzyme.

1443

Optical sensors with an inbuilt PEGylated polymer coat have 1444 also been investigated for in vivo detection of reactive oxygen 1445 species (ROS) and reactive nitrogen species (RNS), hallmarks 1446 of many pathological processes including chronic diseases such 1447 as cancer, cardiovascular disease, and arthritis. An ability to 1448 detect and track these species would both facilitate our 1449 understanding of the etiology of these diseases and support 1450 therapeutic intervention. ${ }^{237}$ With this in mind, Zhen et al. have 1451 reported the development of polymer nanoparticles capable of 1452 the sensitive in vivo detection of hydrogen peroxide $\left(\mathrm{H}_{2} \mathrm{O}_{2}\right){ }^{238}{ }_{1453}$ These multifunctional nanoparticles were generated by nano- 1454 precipitation of a PEG polymer network (subsequently an 1455 antifouling shell), a semiconducting polymer (SP), and the 1456 chemiluminescent substrate peroxalate bis(2,4,6-trichlorophen- 1457 yl) oxalate (TCPO). The amphiphilic PEG-b-PPG-b-PEG 1458 triblock polymer served as a matrix for the encapsulation of SP 1459 and TCPO while also endowing the particles with good fouling 1460 resistance, water-solubility, and biocompatibility. The sensor 1461 showed excellent colloidal stability in aqueous solution as no 1462 precipitation or change in size observed after two months. 1463 Doping of the SP with a naphthalocyanine dye leads to 1464 intraparticle chemiluminescence resonance energy transfer 1465 (CRET), resulting in near-infrared (NIR) luminescence in 1466 the presence of $\mathrm{H}_{2} \mathrm{O}_{2}$. The circulating nanocomposites $(\sim 101467$ $\mathrm{nm}$ ) were shown to detect $\mathrm{H}_{2} \mathrm{O}_{2}$ down to $5 \mathrm{nM}$ in living mice 1468 and to exhibit a chemiluminescence quantum yield significantly 1469 higher than that of previous nanoparticle probes. Importantly, 1470 


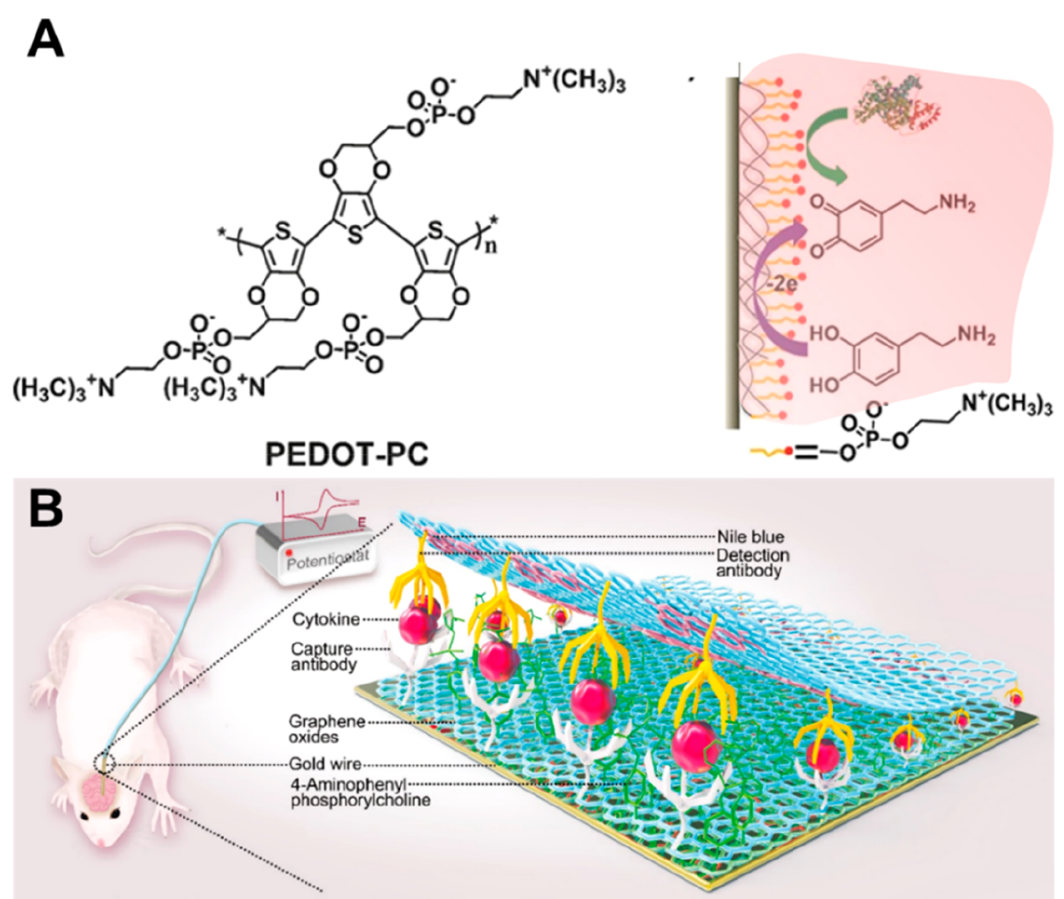

Figure 24. (A) Structure of PEDOC-PC and schematic of the interface between PEDOC-PC/CFE and bloodstream. Adapted with permission from ref 244. Copyright 2017 John Wiley and Sons. (B) Schematic depiction of zwitterionic PPC-GO decorated sensor for electrochemical sandwich sensing of IL-6 cytokine in a mouse brain. Reproduced with permission from ref 245. Copyright 2017 American Chemical Society.

1471 these NIR probes with CRET capability sense $\mathrm{H}_{2} \mathrm{O}_{2}$ without 1472 the need of external light excitation. ${ }^{238}$

1473 Shuhendler et al. have also reported the use of a 1474 functionalized PEG polymer within an optical nanosensor for 1475 the simultaneous detection of drug (e.g., antipyretic 1476 acetaminophen (APAP)) induced RNS and ROS in the liver 1477 of living mice to support the evaluation of acute hepatotoxicity 1478 in real time. ${ }^{239}$ This sensor (average diameter $\sim 50 \mathrm{~nm}$ ) is also 1479 a nanocomposite generated by coprecipitation, in which the $1480 \mathrm{H}_{2} \mathrm{O}_{2}$-sensitive chemiluminescent compound bis(2,4,5-tri1481 chloro-6-(pentyloxycarbonyl)phenyl)oxalate (CPPO) and a 1482 ROS-sensitive fluorescent dye (IR775 S) are integrated into a 1483 PEG-polymer. The CPPO enabled the detection of $\mathrm{H}_{2} \mathrm{O}_{2}$ 1484 without external light excitation via CRET, and the IR775 S 1485 facilitated ratiometric fluorescence resonance energy transfer 1486 (FRET) detection of $\mathrm{ONOO}^{-}$upon light excitation. The 1487 nanosensors were injected intravenously through a tail-vein 1488 catheter $15 \mathrm{~min}$ after drug administration, after which imaging 1489 of the liver using an IVIS Spectrum imaging system was 1490 performed. The authors assessed the rate of nanosensor uptake 1491 and retention in the liver by measurement of the total liver 1492 fluorescence intensity for $80 \mathrm{~min}$. The sensor exhibited specific 1493 and nanomolar sensitivity toward oxidative and nitrosative 1494 species generated during triggered pathophysiological con1495 ditions.

1496 Xie et al. have described the construction of a PEG 1497 containing nonfouling flower-shaped $\mathrm{Au}-\mathrm{Fe}_{3} \mathrm{O}_{4}$ based nanop1498 robe $\left(D_{\mathrm{h}} \sim 40 \mathrm{~nm}\right.$ ideally define for all particle cases) that can 1499 be injected into mice for optically sensing matrix metal1500 loproteinase (MMP) expression in vivo. ${ }^{240}$ MMP is a protease 1501 related to tumor invasion and metastasis and is heavily secreted 1502 by malignant tumor cells, making it a valuable biomarker of 1503 cancer. As illustrated in Figure 23, the presynthesized $\mathrm{Au}-$ $1504 \mathrm{Fe}_{3} \mathrm{O}_{4}$ nanoparticles were modified with a Cy5.5-labeled 1505 GPLGVRG peptide containing a tridihydroxyphenylalanine
(TDOPA) anchor (Cy5.5-GPLGVRG-TDOPA), and PEG $5000^{-} 1506$ $\mathrm{SH}$. Cy5.5 serves as a NIR optical reporter, which is linked to 1507 the particle by the enzyme substrate (GPLGVRG) spacer. 1508 Because of the quenching of the Cy5.5 emission by the 1509 underlying gold substrate, the fluorescence of the sensor is 1510 turned off in its native state. Upon cleavage of the peptide 1511 spacer by MMP, the fluorophore is released and the 1512 fluorescence restored. The nanoprobes were injected intra- 1513 venously in SCC-7 (head and neck squamous cell carcinoma) 1514 tumor xenograft mouse models, which are known to express 1515 high levels of MMP, and subsequent NIR imaging demon- 1516 strated a 10-times stronger signal than control experiments in 1517 which a MMP inhibitor was used. Notably, almost no 1518 fluorescence signal was observed in blood while control 1519 particles without PEG were strongly fluorescent due to 1520 nonspecific activation of the sensor upon fouling. ${ }^{106,240} 1521$ Importantly, this platform could also be applicable to detect 1522 other proteases implicated in cancer pathogenesis if their 1523 respective enzymatic substrates are incorporated into this 1524 platform.

1525

Despite these promising works, it should be noted that 1526 recent reports indicate that PEG may induce immunogenicity 1527 and subsequently produce anti-PEG antibodies, which may 1528 limit the application of PEG coatings for in vivo sensing or drug 1529 delivery, prompting the exploration of other antifouling 1530 chemistries (Table 4). ${ }^{241-243}$

1531

2.3.3.2. Zwitterions. In taking advantage of the discussed 1532 properties of zwitterionic interfaces, Liu et al. have recently 1533 reported an amperometric sensor for in vivo tracking of DA by 1534 electropolymerizing a EDOT-PC composite on carbon fiber 1535 electrode (CFEs) (Figure 24A). ${ }^{244}$ Fast scan CV was then used 1536 f24 to record the electrically stimulated DA release in the rat 1537 nucleus accumbens. Notably, the authors demonstrated that 1538 the amperometric response toward DA was almost completely 1539 maintained at the PEDOT-PC/CFE after in vivo implantation 1540 
Table 3. Summary of Antifouling Durable (Implantable) Sensors ${ }^{a}$

\begin{tabular}{|c|c|c|c|c|c|c|}
\hline antifouling chemistry & sensor & detection mode & operation site & lifetime & analyte & ref \\
\hline PEG & CFE & voltammetry & rat brain & $\sim 1$ year & dopamine & 226 \\
\hline PEG & $\begin{array}{l}\text { semiconducting polymer nanoparticle based } \\
\text { nanosensor }\end{array}$ & CRET & mouse peritoneal cavity & $\sim 4 \mathrm{~h}$ & $\mathrm{H}_{2} \mathrm{O}_{2}$ & 238 \\
\hline PEG & $\mathrm{Au} @ \mathrm{Fe}_{3} \mathrm{O}_{4}$ based nanosensor & fluorescence & mouse venous blood & $\sim 8 \mathrm{~h}$ & MMP & 240 \\
\hline PEG & organosilica core-shell NP based nanosensor & fluorescence & mouse venous blood & $\sim 15 \mathrm{~min}$ & $\mathrm{pH}$ & 233 \\
\hline PEG & carbon dot based nanosensor & fluorescence & mouse vein & $\sim 10 \mathrm{~d}$ & cancer cell & 251 \\
\hline PEG & $\begin{array}{l}\text { semiconducting polymer nanoparticle based } \\
\text { nanosensor }\end{array}$ & FRET & mouse vein & $\sim 24 \mathrm{~h}$ & RONS & 252 \\
\hline PEG & DNA-CNT based nanosensor & fluorescence & mouse vein & $\sim 40 \mathrm{~h}$ & $\mathrm{NO}$ & 253 \\
\hline PEG & Pt nanosensor & phosphorescence & mouse venous blood & $\sim 2 \mathrm{~h}$ & histamine & 235 \\
\hline PEG & $\begin{array}{l}\text { semiconducting polymer dot based } \\
\text { nanosensor }\end{array}$ & FRET & mouse vein & $\sim 24 \mathrm{~h}$ & cancer cell & 254 \\
\hline PEG & hydrogel microbeads & fluorescence & mouse vein & $\sim 3.5 \mathrm{~h}$ & glucose & 218 \\
\hline OEG & gold electrode & EIS & mouse brain & $\sim 6 \mathrm{~min}$ & $\mathrm{~K}^{+}$ & 231 \\
\hline cysteine & CdTeS QD based nanosensor & FRET & mouse venous blood & $\sim 36 \mathrm{~h}$ & MMP-2 & 250 \\
\hline PEOT-PC & $\mathrm{CFE}$ & amperometry & rat striatum & $\sim 50 \mathrm{~min}$ & dopamine & 244 \\
\hline Phosphatidylcholine & gold wire & amperometry & rat vein & $\sim 3 \mathrm{~h}$ & doxorubicin & 246 \\
\hline PVA-CS & silicon microneedle & colorimetry & rabbit skin & $\begin{array}{l}\sim 17 \\
\quad \text { days }\end{array}$ & glucose & 255 \\
\hline $\begin{array}{l}\text { silica nanoporous } \\
\text { membrane }\end{array}$ & CFE & amperometry & rat brain & $\sim 2 \mathrm{~h}$ & $\mathrm{O}_{2}$ & 256 \\
\hline ion-selective membrane & CFE & potentiometry & rat brain & $\sim 50 \mathrm{~min}$ & $\mathrm{pH}$ & 257 \\
\hline $\begin{array}{l}\text { nanoporous polymer } \\
\text { membrane }\end{array}$ & CFE & amperometry & rat brain & $\sim 1 \mathrm{~h}$ & dopamine & 258 \\
\hline $\begin{array}{l}\text { continuos-flow diffusion } \\
\text { filter }\end{array}$ & CFE & SWV & rat jugular vein & $\sim 4 \mathrm{~h}$ & doxorubicin & 259 \\
\hline polysulfone membrane & gold wire & amperometry & rat external jugulars & $\sim 12 \mathrm{~h}$ & doxorubicin & 260 \\
\hline Nafion membrane & Pt wire & amperometry & $\begin{array}{l}\text { rabbit femoral quadriceps } \\
\text { muscle }\end{array}$ & $\sim 15 \mathrm{~min}$ & $\mathrm{O}_{2}$ & 261 \\
\hline Nafion membrane & CFE & amperometry & rat brain & $\sim 6 \mathrm{~h}$ & dopamine & 262 \\
\hline
\end{tabular}

a Abbreviations: CFE, carbon-fiber electrode; PEDOT-PC, polyethylenedioxythiophene-phosphorylcholine; PVA, poly(vinyl alcohol); CS, chitosan; MPA, 3-mercaptopropionic acid; FSCV, fast-scan cyclic voltammetry; MMP, matrix metalloproteinase; CRET, chemiluminescence resonance energy; RONS, reactive oxygen and nitrogen species; FRET, fluorescence resonance energy transfer.

1541 in the striatum of the rat brain compared to in vitro detection 1542 in cerebral spinal fluid (CSF), indicating a high resistance to 1543 nonspecific adsorption. Moreover, DA could be monitored 1544 within $400 \mathrm{~s}$ after local injection of $\mathrm{KCl}$ (to evoke DA release) 1545 with good sensitivity. In a recent study, Qi et al. presented 1546 another phosphorylcholine decorated electrochemical sensor 1547 for amperometric monitoring secretion of Interleukin-6 (IL-6, 1548 a major cytokine in the central nervous system) in mouse 1549 brain. $^{245}$ They introduced a sandwich assay consisting of a 1550 capture antibody and electrografted zwitterionic PPC-modified 1551 graphene oxide on gold wire electrodes together with a 1552 detection antibody and Nile blue-modified GO (Figure 24B). 1553 In in vitro tests, this platform was capable of the highly selective 1554 amperometric detection of IL-6 down to sub pM levels. The 1555 developed sensor was also capable of monitoring IL-6 secretion 1556 in live mice after intraperitoneal injection of lipopolysaccharide 1557 (LPS), without inducing an inflammatory response. ${ }^{245}$ 1558 Although this study was not designed for continuous real 1559 time and long-term monitoring, it presents a pioneering 1560 exploration in translating in vitro surface chemistry design 1561 (zwitterion-GO composite) into an in vivo application.

1562 Another phosphatidylcholine (PC) based electrochemical in 1563 vivo sensor has been reported by the Plaxco group, who 1564 utilized mixed SAMs of a biomimetic PC-thiol and a 1565 methylene-blue tagged aptamer for sensing of doxorubicin in 1566 the external jugular vein of an anesthetized rat. ${ }^{246}$ The working 1567 principle of this system is akin to in vitro aptamer sensors 1568 discussed in Section 2.3.2.4 (Figure 21), where target binding triggers conformation changes that affect electric communica- 1569 tions between a redox-labeled capture probe and the 1570 underlying electrode. ${ }^{247,248}$ The incorporation of the cell- 1571 membrane mimetic PC antifouling system allowed successful 1572 translational to in vivo monitoring of doxorubicin (DOX). On 1573 the basis of this system, the authors demonstrated significantly 1574 improved baseline stability, with a stable current response over 1575 $12 \mathrm{~h}$. In contrast, control interfaces based on $\mathrm{MCH}$ or 1- 1576 mercapto-1-undecanol (MCU) diluents suffered significant 1577 signal loss due to fouling. This platform thus enabled a stable 1578 real-time monitoring of pharmacokinetic information, poten- 1579 tially of value in disease management. ${ }^{249}$

1580

The discussed physicochemical characteristics of amino acid 1581 interfaces have been exploited in a recent study, where a 1582 cysteine-modified quantum dot based FRET nanosensor was 1583 developed by $\mathrm{Li}$ and co-workers to detect matrix metal- 1584 loproteinase-2 (MMP-2) in vivo. ${ }^{250}$ Specifically, cysteine- 1585 decorated NIR emitting $(720 \mathrm{~nm})$ CdTeS QDs (donor) 1586 were linked to an NIR organic dye (ICG-Der02, acceptor) via 1587 a MMP-2-specific peptide substrate (GPLGVRGKGG) to 1588 form a QD-peptide-dye nanoassembly. Exposure of the 1589 sensor to MMP-2 in the tumor environment via intratumoral 1590 injection led to selective cleavage of the peptide (due to 1591 specific recognition by the MMP-2), resulting in the recovery 1592 of fluorescence from QDs. These particles were reported to 1593 show high colloidal stability with no precipitation in MCF-7 1594 cancer cell medium. The authors then successfully examined 1595 MMP-2 expression in tumors on nude mice noting a clear 1596 
1597 difference between tumor and symmetrical muscles in the two 1598 upper arms as tracked by fluorescence for up to $36 \mathrm{~h}$.

1599 In summary, in vivo sensors need to satisfy the target 1600 selective criteria that in vitro sensors must operate with when 1601 applied within (usually diluted) biological samples but 1602 additionally require high levels of biocompatibility, low 1603 toxicity, and need to be miniaturizable/machinable to enable 1604 effective operation in harsh, spatially confined and often 1605 dynamic conditions. The presented examples largely rely on 1606 the use of an appropriate combination of a solid support (e.g., 1607 QDs, fiber or microelectrodes with an antifouling protection, 1608 often PEG-based). The application of these designs can not 1609 only minimize fouling but also decrease host immunoresponse 1610 and thrombus formation and can stabilize colloidal nano1611 sensors as well as reduce their clearance (thus increasing the 1612 sensor lifetime). Though the highlighted examples hold great 1613 promise for monitoring internal body dynamics, and thus 1614 supporting both health management and disease theranostics, 1615 routine applications remain some way off. More detailed 1616 studies are required to assess the long-term stability, safety, and 1617 biocompatibility of these in vivo agents, but there remains 1618 enormous bandwidth in chemical and physical tailoring (as 1619 detailed in Sections 2 and 4) in a manner that might, 1 day 1620 support routine automated monitoring of a wide range of 1621 relevant analytes. Of particular importance in the design of in 1622 vivo sensors is their lifetime, as highlighted throughout this 1623 review (see, for example, Tables 1 and 3 ). The requirements 1624 here are very application specific; the monitoring of 1625 pharmacokinetics is typically envisioned to be over compara1626 tively short time spans (hours to days), while, for example, 1627 continuous glucose sensing may be sought over many weeks. 1628 The challenges of long-term stability and antifouling character 1629 scale, of course, with time but are also a factor of the analytical 1630 mode. In "reagentless" or immunorecognition configurations, 1631 this can be a very substantial challenge, markedly more so than 1632 when analyte capture and signal generation is enzymic. As 1633 indicated in Table 3, there remains much work to be done 1634 before academic work can be robustly translated into clinical 1635 application.

\section{SENSORS BASED ON PHYSICAL ANTIFOULING STRATEGIES} 1636

\subsection{Porous Transducer Topographies}

1637 Apart from the previously discussed sensors that rely on highly 1638 hydrated interfacial chemistries to resist nonspecific adsorp1639 tion, alternative strategies to alleviate the impediments 1640 associated with interfacial fouling have also been explored. 1641 One such approach is the direct physical manipulation of 1642 sensory interfaces. For example, the engineering of porous 1643 electrodes or the use of membrane filters are promising 1644 approaches to alleviate fouling by large molecules or cells by 1645 presenting a diffusional barrier (sieving) that restricts access of 1646 these large molecules to the underlying sensor while still 1647 allowing diffusive access to smaller analytes. To this end Patel 1648 et al. have explored the relationship between electrode pore 1649 size and antifouling performance (Figure 25A). ${ }^{263}$ In this work 1650 it was found that nanoporous ( $<50 \mathrm{~nm}$ pore size) electrodes 1651 possess greater fouling resistance compared to planar, 1652 macroporous (1200 nm pore size) or hierarchical (60 nm16531200 pore size) gold electrodes in the presence of BSA (2 mg $\left.1654 \mathrm{~mL}^{-1}\right)$ and fibrinogen $\left(1 \mathrm{mg} \mathrm{mL}^{-1}\right)$. Cyclic voltammetry in a 1655 solution containing a redox probe $\left(\left[\mathrm{Fe}(\mathrm{CN})_{6}\right]^{3-/ 4-}\right)$ indicated
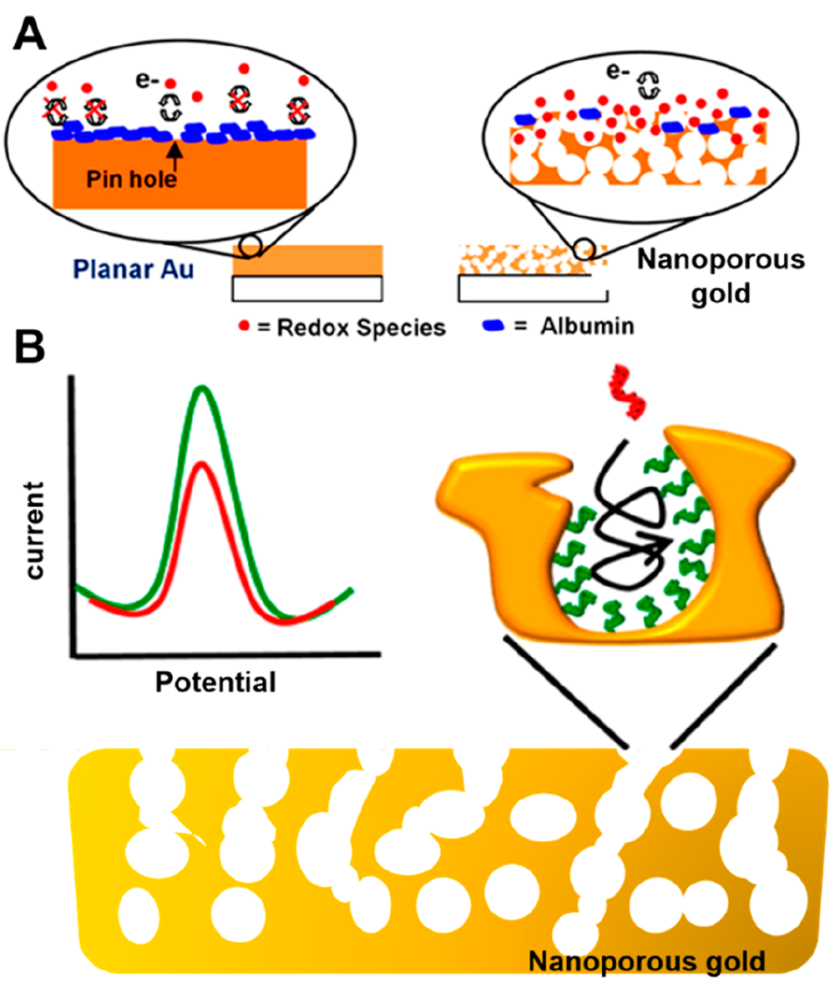

Figure 25. (A) Graphical representation of the surface of nanoporous gold and planar gold in the presence of albumin and a small redox molecule in solution. Electron transfer is hindered at planar gold but not at nanoporous gold. Reproduced with permission from ref 263. Copyright 2013 American Chemical Society. (B) Schematised summary of the use of a porous gold thin film morphology for electrochemical DNA Sensing. Adapted with permission from ref 264. Copyright 2015 American Chemical Society.

negligible fouling at the nanoporous electrode. It was proposed 1656 that restricted mass transport through small pores was key to 1657 this attenuation, an operational feature that can be feasibly 1658 exploited for sensing of small analytes.

1659

The same group investigated a similar strategy that utilizes 1660 porous gold electrodes obtained via dealloying. ${ }^{263}$ No 1661 significant loss in voltammetric response of ferricyanide was 1662 observed when the nanoporous gold (np-Au) was immersed in 1663 serum or heparinized blood while a significant reduction in 1664 Faradaic peak currents occurred at planar gold. The high 1665 receptive loading possible with these large surface area 1666 supports has also been shown to enable an approximately 1667 10-fold higher electrochemical current upon probe-target 1668 hybridization as compared to the planar Au electrodes (Figure 1669 25B). ${ }^{264}$ On the basis of these discoveries, the Seker group 1670 have applied porous gold based sensors for assaying DNA in 1671 complex biological media. ${ }^{264-266}$ In one example, they 1672 reported using $\mathrm{np}$-Au electrodes onto which MB functionalized 1673 DNA probes where self-assembled. This allowed detection of 1674 target DNA in the presence of BSA and FBS. ${ }^{266}$ The np-Au 1675 electrodes enabled sensitive detection with a dynamic range of 1676 10 to $100 \mathrm{nM}$ that improves by 1 order of magnitude for 1677 coarsened np-Au morphology due to increased target 1678 penetration into the porous network and hence enhanced 1679 hybridization efficiency. In comparison with planar $\mathrm{Au}$ and 1680 annealed $\mathrm{np}-\mathrm{Au}$ electrodes, unannealed porous Au electrodes 1681 suffered least signal suppression in the presence of BSA or FBS, 1682 with highest suppression observed at planar $\mathrm{Au}$ electrodes 1683 
1684 indicating that the pores act as efficient sieves for larger 1685 (fouling) biomolecules. Importantly, a tuning of the pore size 1686 may allow the design of sensors in which molecules of specific 1687 sizes are restricted from accessing the sensory (electrode) 1688 interface. Nanoporous gold has also been applied to electro1689 chemical detection of L-cysteine in urine ${ }^{267}$ while in another 1690 recent study, simultaneous amperometric electrochemical 1691 sensing of ascorbic acid and uric acid in FBS was achieved 1692 with a LoD of 63.0 and $9.0 \mu \mathrm{M}$, respectively. ${ }^{268}$

\subsection{Filtration Methodologies}

1693 An alternative physical approach, the application of membrane 1694 filters to transducer, has received considerable attention 1695 recently. For example, a drop-cast nanocomposite of graphene 1696 nanoribbons and a Nafion-modified electrode for the selective 1697 detection of cysteine in serum, was reported by Wu et al. ${ }^{269}$ 1698 The graphene nanoribbons impart high conductivity and 1699 extremely highly electrocatalytic activity toward the electro1700 chemical oxidation of cysteine at $+0.025 \mathrm{~V}$ while the negatively 1701 charged Nafion can not only apparently prevent the 1702 aggregation of graphene nanoribbons, but also prevent 1703 electroactive interference from ascorbic acid and uric acid 1704 allowing an in vitro assay of cysteine in serum samples without 1705 any pretreatment. A different filtering approach was utilized by 1706 Su's group in preparing isoporous silica micelle membranes 1707 (iSMM) and used them for the electrochemical detection of 1708 drug molecules (chloramphenicol, CAP) in 10\% heparinized 1709 whole blood. ${ }^{270}$ The interfaces were formed by immersion of 1710 ITO into tetraethoxysilane solution forming small channels in 1711 the silica support that facilitate access of small molecules to the 1712 underlying electrode while blocking (sieving) large biointerfer1713 ents, preventing fouling and passivation. By monitoring CAP's 1714 direct reduction peak current using DPV, this electrochemical 1715 sensor enabled the quantification of CAP in whole blood with 1716 a $100 \mathrm{nM}$ LoD substantially lower than that achieved at a bare 1717 ITO electrodes.

1718 Membrane filtration based sensors have also been applied 1719 for in vivo sensing. For example, for the sensitive in vivo 1720 monitoring of $\mathrm{pH}$ (important marker for physiological 1721 function) in a live rat brain, ${ }^{271}$ Mao's group developed a $\mathrm{H}^{+}$ 1722 selective electrode based on a carbon fiber coated with a 1723 polyvinyl chloride $(\mathrm{pVC})$ based $\mathrm{H}^{+}$selective membrane $\left(\mathrm{H}^{+}\right.$ 1724 ISM) containing the $\mathrm{H}^{+}$ionophore tridodecylamine. ${ }^{257}$ Both in 1725 vitro and in vivo studies demonstrated that the resultant 1726 membrane-coated potentiometric $\mathrm{pH}$ sensor possessed strong 1727 antifouling properties against protein adsorption without loss 1728 of sensitivity when exposing to BSA (up to $70 \mathrm{mg} \mathrm{mL}^{-1}$ ) or 1729 cerebrospinal fluid (CSF) containing $40 \mathrm{mg} \mathrm{mL}^{-1}$ BSA. 1730 Moreover, this sensor was reported to exhibit a fast response $1731(<1 \mathrm{~s})$ to $\mathrm{pH}$ change within the narrow physiological $\mathrm{pH}$ range 1732 ( $\mathrm{pH} 6.0$ to 8.0 ) with high reversibility and selectivity when 1733 animals were subjected to $\mathrm{CO}_{2}$ inhalation or injection of 1734 sodium bicarbonate to evoke acid-base disturbance. The 1735 translation of such ISMs for in vivo monitoring of other ions 1736 could be achieved if suitable ionophores are incorporated into 1737 these architectures. Recently, Maós group demonstrated in vivo 1738 monitoring of oxygen in rat brain with carbon fiber 1739 microelectrode (CFME)-modified with an antifouling silica 1740 nanoporous membrane. Specifically, a silica nanoporous 1741 membrane (SNM) consisting of uniform, closely packed, and 1742 vertically aligned nanochannels was electrografted (from a 1743 silane solution containing CTAB as a surfactant) onto the 1744 CFME surface, which was permeable to $\mathrm{O}_{2}$ while nonfouling
(Figure 26). ${ }^{256}$ Continuous monitoring of $\mathrm{O}_{2}$ for up to $2 \mathrm{~h}$ was $1745 \mathrm{f} 26$ achieved with this sensor in the mouse brain with retained 1746

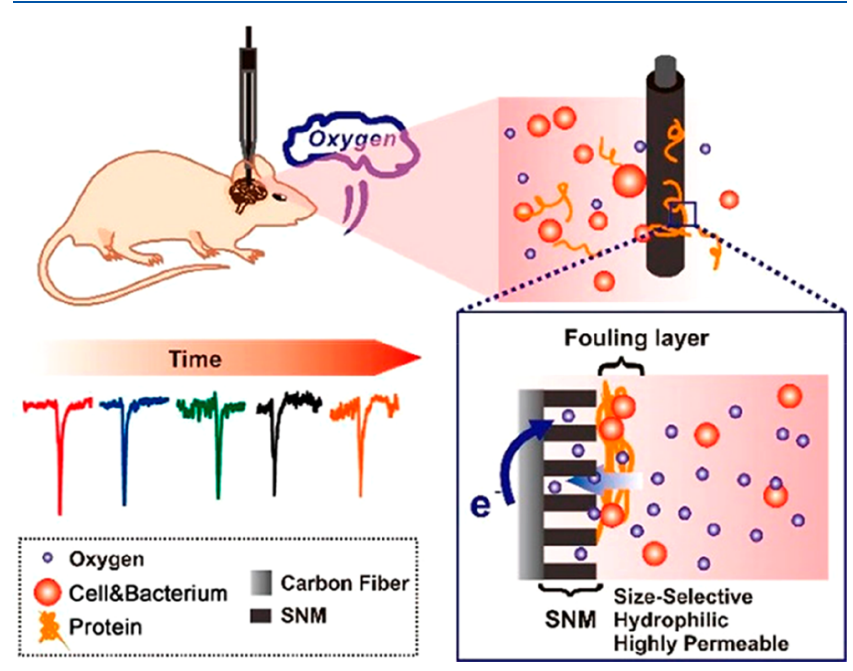

Figure 26. Illustration of hydrophilic, highly permeable and antibiofouling SNM-coated CFME for continuous monitoring of $\mathrm{O}_{2}$ in rat brain. Reproduced with permission from ref 256. Copyright 2019 American Chemical Society.

current stability. A similar strategy using silica porous 1747 membrane has also been applied as antifouling coating for 1748 electrochemical sensing of dopamine ${ }^{272}$ or chloramphenicol ${ }^{273} 1749$ in human blood.

1750

Another filtration-based methodology to prevent electrode 1751 surface biofouling has been utilized by Soh and Plaxco to 1752 facilitate the amperometric electrochemical sensing of small 1753 molecules. $^{259}$ In an example of an off-surface filter, a 1754 continuous monitoring of the pharmacokinetics of DOX, was 1755 enabled. A "continuous diffusion filter" (CDF) was specifically 1756 utilized to block access of large molecules or blood cells while 1757 enabling diffusion of small analytes to the underlying electrode 1758 (Figure 27). Amperometric detection was achieved through $1759 \mathrm{f} 27$ the use of a conformationally responsive DOX binding 1760 methylene blue-tagged specific aptamer. It was demonstrated 1761 that this sensor allowed continuous DOX monitoring in live 1762 rats over $4.5 \mathrm{~h}$ with good sensitivity and a highly selective 1763 performance ultimately relying on the $\sim 100$ times higher 1764

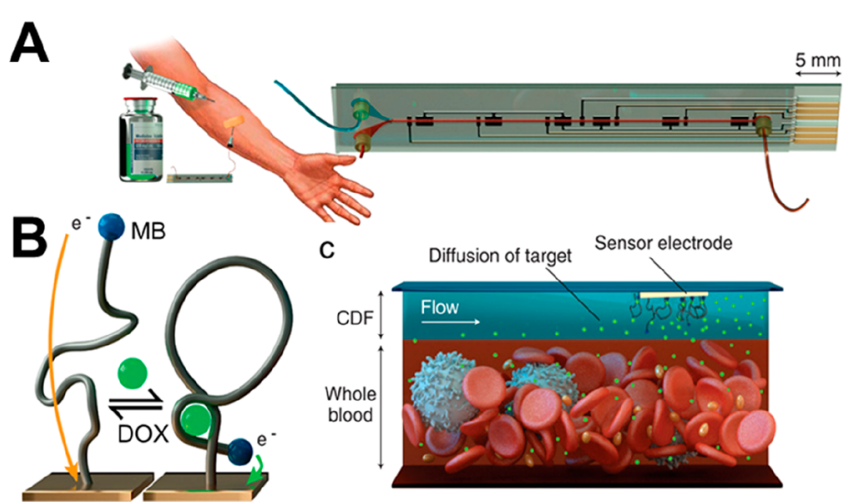

Figure 27. Microfluidic electrochemical sensor incorporating a continuous diffusion filter (CDF) allowing real-time quantitative measurement of small molecules in the blood flow of humans. Adapted with permission from ref 259. Copyright 2017 American Association for the Advancement of Science (AAAS). 
1765 diffusivity of the DOX analyte in comparison to background 1766 HSA, IgG and Fib. Though conceptually simple, and 1767 inherently limiting, this strategy might be generally applicable 1768 to the sensing of a wide range of small-molecule analytes. In a 1769 recent study, the Plaxo group applied an alternative approach 1770 to produce an electrode that could be inserted directly into a 1771 catheter to monitor blood flow in the jugular vein. ${ }^{260}$ Rather 1772 than using the off-surface filter, the authors integrated a 1773 biocompatible polysulfone membrane $(0.2 \mu \mathrm{m}$ pore size $)$ onto 1774 the electrode. The system was used to measure the real-time 1775 concentrations and pharmacokinetics of DOX and several 1776 aminoglycoside antibiotics (intravenous or intramuscular 1777 injection) using redox-tagged aptamer films. The sensor was 1778 able to identify pharmacokinetic characteristics across multiple 1779 injection cycles and on both awake and mobile rats. These in 1780 vivo sensing systems are applicable for many small molecule 1781 therapeutic drugs but are ultimately limited to the detection of 1782 analytes that can penetrate through the filtering membrane. It 1783 is also highly unlikely this purely physical approach would be 1784 effective as coexisting nontarget small molecules may also 1785 access and disturb the sensing interface. Taking this into 1786 account, the concurrent integration of a chemically antifouling 1787 system in a manner perhaps analogous to the zwitterionic 1788 membranes, which have been widely applied in water 1789 desalination, might further improve these systems. ${ }^{274}$ Such 1790 an approach was recently reported by Feng et al., who utilized 1791 a polytannic acid-doped nanoporous conductive PANI 1792 membrane on CFEs through potential-static electropolymeri1793 zation. $^{258}$ The concurrent integration of physical (porous 1794 membrane) and chemical antifouling strategies (polytannic 1795 acid/PANI) resulted in excellent antifouling performance, 1796 enabling in vivo DA quantification in mouse brain, where high 1797 sensitivity was retained pre- and postcalibration.

1798 These physical antifouling approaches present (Table 3) a 1799 promising new approach to circumvent fouling by restricting 1800 access of large biomolecules to the underlying sensory 1801 interface. They are, however, inherently restricted to the 1802 sensing of small analytes. Through the tuning of the pore size 1803 of a filter or the porous support, a relatively distinct cutoff size/ 1804 molecular weight can be achieved. This will not only reduce 1805 any fouling from larger molecules, but also inherently impact 1806 the selectivity of the sensor in a manner that needs to be 1807 carefully considered.

\section{SENSORS BASED ON BIOLOGICAL ANTIFOULING APPROACHES}

\subsection{Affinity Depletion}

1809 Another approach to the detection of analytes in complex 1810 samples is off-sensor depletion. In contrast to chemical and 1811 physical strategies, this methodology serves to directly reduce 1812 the concentration of interfering (bio)molecules. Such a sample 1813 prepurification can also be carried out via other "conventional" 1814 techniques such as liquid chromatography, although these are 1815 time-consuming or considerably dilute the sample. To 1816 circumvent this, antibody-modified magnetic beads can 1817 directly and conveniently capture and remove abundant 1818 coexisting proteins, such as HSA or IgG in blood, thereby 1819 reducing the fouling capabilities of the sample. For example, 1820 Kongsuphol et al. have used two types of magnetic beads 1821 (MBs) decorated with anti-HSA/anti-IgG and anti-TNF- $\alpha .^{275}$ 1822 The former was used to deplete highly abundant HSA and IgG 1823 from serum to generate precleared serum, followed by specific capture of TNF- $\alpha$ by the latter beads (Figure 28). The $1824 \mathrm{f} 28$ captured TNF- $\alpha$ was then eluted from the beads and assayed 1825

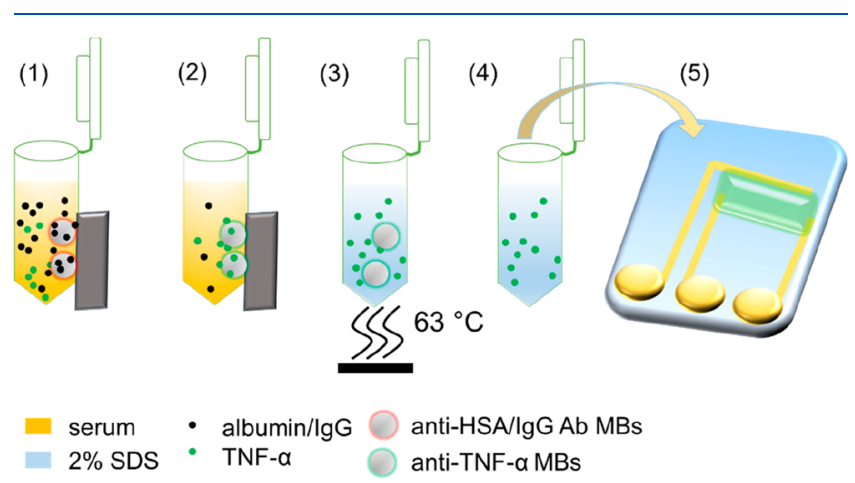

Figure 28. Schematic depiction of a TNF- $\alpha$ impedimetric sensor with an integrated background depletion strategy. (1) Removal of the main serum background interferents (HSA and IgG). (2) The background depleted sample is collected for TNF- $\alpha$ capture. (3) The captured TNF- $\alpha$ is eluted from the magnetic beads using $2 \%$ sodium dodecyl sulfate (SDS) at $63{ }^{\circ} \mathrm{C}$ and (4) the magnetic beads are separated from the eluted sample. (5) Eluted TNF- $\alpha$ is detected at gold array electrode via EIS. Adapted with permission from ref 275. Copyright 2014 Elsevier.

by impedance spectroscopy at bare gold microelectrode arrays 1826 in a manner that was reportedly able to detect TNF- $\alpha$ with a 1827 $\mathrm{fM}$ sensitivity in undiluted serum samples. The assay is small 1828 sample volume only as the total protein level in serum $(\sim 50-1829$ $\left.60 \mathrm{mg} \mathrm{mL}^{-1}\right)$ is usually significantly higher than specific target 1830 concentrations requiring large input quantities of antibody- 1831 modified depletion beads (Table 4) and this remains a time- $1832 \mathrm{t} 4$ consuming two-step process. It does, though, allow specific 1833 depletion and could be combined with traditional chemical 1834 nonfouling approaches (i.e., decoration of bead/sensory 1835 interface with antifouling chemistries). It is also likely to be 1836 more efficient if applied within an integrated fluidic device 1837 where more efficient (and designed) mixing of low sample 1838 volumes is possible. An early example of this was reported by 1839 Oleschuk and co-workers who developed a microfluidic 1840 platform in which three depletion beads (anti-HSA, protein 1841 $A$, and protein $G$ ) purified up to four human serum samples 1842 simultaneous depleting up to $95 \%$ of IgG and HSA within 101843 min. This enabled a 4-fold increase in signal-to-noise ratio in 1844 the subsequent mass spectrometric detection of hemopexin, a 1845 low abundance protein biomarker. ${ }^{276}$

1846

\subsection{Degradation by Enzyme Catalysis}

An entirely different biological antifouling strategy has been 1847 developed based on the use of enzymes capable of actively 1848 degrading (physisorbed) biomolecules thus reducing fouling. 1849 This has been widely utilized for the modification of 1850 membranes utilized in the water treatment industry. ${ }^{277,278} 1851$ For example, in the construction of an antifouling and self- 1852 cleaning membrane based on the covalent attachment of 1853 trypsin on a poly(methacrylic acid)-graft-poly(ether sulfone) 1854 (PMAA-g-PES) membrane via EDC/NHS coupling. ${ }^{279}$ This 1855 suppressed protein fouling under dynamic flow where $95.0 \%$ of 1856 the initial flux of BSA solution (through the membrane) was 1857 maintained after multicycle BSA filtration over a 15-day period. 1858 A control PES membrane suffered more significantly from 1859 fouling ( $<35 \%$ flux recovery) within a shorter time span of 501860 h. In another example, Koseoglu-Imer et al. reported the 1861 successful modification of a cellulose acetate membrane with a 1862 


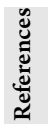

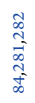

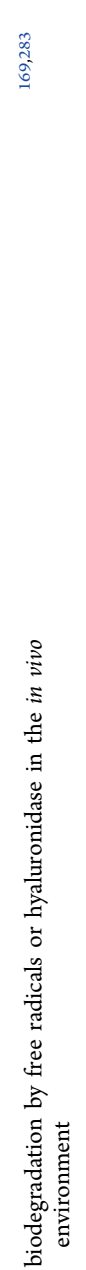

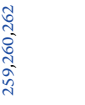

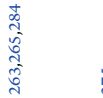
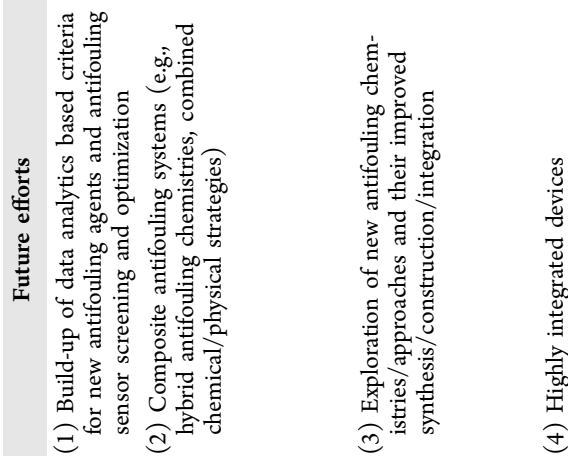

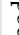

serine protease enzyme (Savinase) in retaining higher flux 1863 value and protein (BSA) rejection during filtration in 1864 comparison to the unmodified membrane. ${ }^{280}$ This approach 1865 could be incorporated into a variety of existing microfluidic 1866 devices or sensors relying on chemical or physical antifouling 1867 approaches. One note of caution is that, in addition to an 1868 appropriate selection and immobilization of the enzyme, the 1869 degradation products must not interfere with the operation of 1870 the sensor.

1871

\section{CONCLUSIONS AND PERSPECTIVES}

The development of nonfouling sensors for the detection of 1872 target analytes in complex fluids has witnessed great progress 1873 in recent years as shown in Figure 29. This review has $1874 \mathrm{f} 29$ attempted to systematically summarize the comprehensive 1875 spectrum on the antifouling interface construction, character- 1876 ization, incorporation strategies with receptor to form final 1877 antifouling sensors, and comment on the key antifouling 1878 strategies that facilitate sensitive and selective sensing under 1879 such demanding conditions (in vitro or in vivo). The chemical 1880 modification of sensory interfaces (flat or spherical) with 1881 suitably designed hydrated molecular systems (such as PEG, 1882 zwitterions or peptides) is undoubtedly an accessible and thus 1883 common approach that is increasing in its sophistication. 1884

Physical or biological approaches to circumvent fouling have 1885 recently gained significant attention as an alternative or 1886 complementary approach to more established chemical 1887 formats. All approaches bring specific advantages to the table 1888 and inherently possess limitations (Table 4) that may have to 1889 be improved if robust real-world applications, at scale, are to be 1890 viable. We thus believe that future efforts within the field will 1891 focus on the following (see also Table 4):

1892

\subsection{Exploration of New Antifouling Materials and} Approaches for Sensing

As discussed within this review, the majority of sensors 1894 operating in complex fluids rely on chemical barriers and 1895 currently these are largely limited to those based on PEG or 1896 accessible zwitterions. Though demonstrably effective, these 1897 have identified specific disadvantages (Table 4), for example 1898 zwitterionic systems can be field- or $\mathrm{pH}$-dependent, peptides or 1899 natural polymers can be prone to hydrolysis (degradation) 1900 while PEG suffers from oxidative damage in the presence of 1901 oxygen and transition and may cause adverse immune 1902 responses in vivo. ${ }^{242}$ It should also be noted that these 1903 problems are especially amplified when continuous, long-term 1904 sensing is carried out or derived devices are expected to have a 1905 tenable shelf life.

1906

The exploration of novel chemical architectures or hybrid 1907 synergic systems as antifouling interfaces is also likely to be 1908 very important as real world applications become increasingly 1909 relevant. This is all the more apparent when considering that in 1910 most applications these interfaces must perform well 1911 diagnostically and be nontoxic. For example, as an alternative 1912 to traditional zwitterions, polyampholytes (polyelectrolytes 1913 comprising mixed charge component polymers) are both 1914 highly tunable and potentially very potent. ${ }^{290}$ Moreover, 1915 zwitterionic inorganic/organic hybrid polymers with high 1916 stability to degradation have been increasingly explored in 1917 the material sciences (for example for the removal of heavy 1918 metal ions from environmental samples) but have not yet been 1919 applied in a sensor format. ${ }^{291,292}$ Inspired by trimethylamine 1920 N-oxide (TMAO), a natural zwitterionic osmolyte, Jiang's 1921 


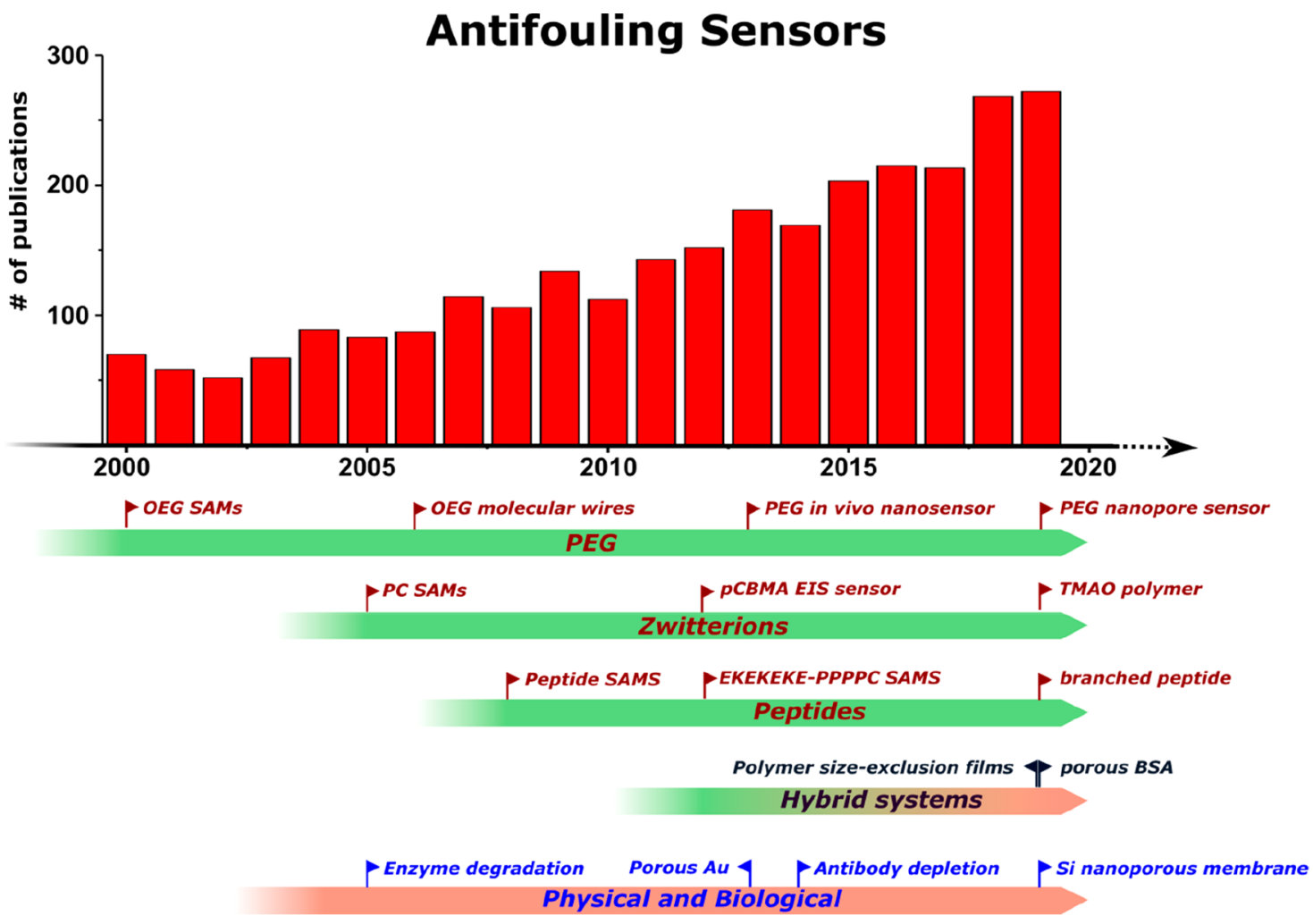

Figure 29. Overview of developments in the field of antifouling sensors. The histogram displays the number of publications per year containing the search terms ("fouling" OR "antifouling" OR "antifouling" OR "nonfouling OR "nonfouling") AND ("sensing" OR sensor" OR "detection") as analyzed by Web of Science (on 24.02.2020). This search found 3116 articles published with these keywords (since 1945) with a total of 79299 citations. Displayed underneath is an approximate timeline of the emergence of the most commonly employed antifouling materials/approaches in the context of sensor development as well as selected milestone examples. These are the introductions of PEG and related systems, ${ }^{142,235,285,286}$ zwitterionic systems, ${ }^{60,102,28}$ peptides, ${ }^{34,168,288}$ hybrid systems ${ }^{117,289}$ as well as physical ${ }^{256,263}$ and biological approaches. ${ }^{275,277}$

1922 group recently developed TMAO-based zwitterionic polymers 1923 (PTMAO) as a new class of ultralow fouling materials with 1924 promising in vitro and in vivo performance. ${ }^{287}$ An interesting 1925 observation with potential ramifications in sensing applications 1926 is a recent report by Yeon et al., demonstrating that 1927 supraparticles (clusters of NPs) decorated with cysteine 1928 moieties of different chirality display a significantly different 1929 interaction with serum proteins (as well as cellular uptake). ${ }^{293}$ 1930 Specifically, the $D$-cysteine-modified particles displayed a 3-4 1931 fold enhanced cell internalization as well as increased stability 1932 against enzymatic degradation and longer biological half-life. 1933 The latter was attributed to the incompatibility of natural 1934 proteins and enzymes with the D-enantiomer-capped particles. 1935 This then potentially offers a new consideration in tuning 1936 biofouling, an approach which may be particularly promising in 1937 peptide based interfaces.

1938 Apart from these chemical antifouling approaches, physical 1939 approaches such as bioinspired topographies, for example, 1940 shark skin patterns ${ }^{268,269}$ or mollusc shell mimics, $190,294,295$ 1941 have been used to resist marine fouling and control cell 1942 adhesion but have, again, rarely found application in sensors. 1943 In another recent study, Yang et al. developed an injectable 1944 antibiofouling "spiky microparticle" where a commercial 1945 fluorosilane was used to entrap a fluorinated lubricant on the 1946 surface. $^{296}$ Interestingly, these particles could be stably 1947 dispersed in water and retained excellent long-term ( $>14$ 1948 days) antiadhesive properties to proteins, cells (both in vitro 1949 and in vivo) and bacterial adsorption (e.g., E. coli and S. 1950 aureus). A similar approach was previously developed by Badv et al. where, in addition to the nonfouling fluorosilanes, 1951 aminosilanes were introduced allowing bioconjugation of cell- 1952 binding ligands or receptors. ${ }^{297}$ A strong adhesion of 1953 endothelial cells with concurrent low levels of fouling in 1954 human whole blood was achieved with the mixed film. These 1955 discoveries highlight the potential of fluorinated, "slippery 1956 surfaces" as a new class of antifouling interfaces with promising 1957 improved or complementary performance to traditional 1958 (hydrated) nonfouling chemistries. ${ }^{298,299} 1959$

In another nice example of a potential new way forward, 1960 Wang et al. have recently reported a proof-of-concept approach 1961 in which electrodes were coated with a commercial transient 1962 polymer (Eudragit L100) for glucose sensing in undiluted 1963 serum and saliva. ${ }^{300}$ This protective and dissolvable coating 1964 can be programmed (by adjusting adlayer density or 1965 thicknesses) to expose fresh sensor surface at preselected 1966 times (up to $6 \mathrm{~h}$ ) (Figure 30). This delayed actuation concept $1967 \mathrm{f} 30$ holds considerable promise for adaption to other electro- 1968 chemical sensing platforms (e.g., sequential activation of 1969 different electrodes within an array) as well as for the 1970 protection of sensing interfaces based on other transduction 1971 modes.

1972

In a very recent example of a combined physical and 1973 chemical approach, Ingber and co-workers have reported the 1974 generation of a cross-linked BSA-nanowire composite interface 1975 with apparent excellent long-term antifouling performance. ${ }^{289} 1976$ The cross-linked BSA matrix in this work presents not only a 1977 strongly hydrated interface but also acts as a physical porous 1978 filter preventing (fouling) access to the embedded electrode 1979 


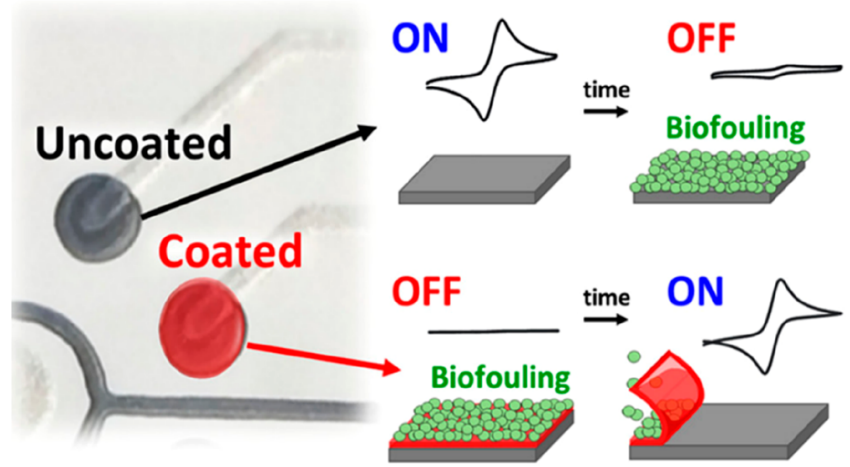

Figure 30. Antibiofouling approach using a delayed sensor activation system exploiting a dissolvable coating which can expose fresh sensor surface at designed times and thus actively remove adsorbed interferents. Reproduced with permission from ref 300. Copyright 2018 American Chemical Society.

1980 materials. This was exploited to indirectly sense IL-6 in human 1981 plasma. Composite interfaces such as these are likely to offer 1982 much to the development of scalable in vitro and in vivo 1983 sensory applications.

1984 Another similar example was reported by Paloni et al., in 1985 which immobilized lamellae of protein-polymer conjugates 1986 (based on poly $(N$-isopropylacrylamide) (PNIPAM)) were able 1987 to allow the access (and subsequent detection) of the small 1988 monomeric streptavidin $(15.6 \mathrm{kDa})$ to the embedded receptive 1989 sites, while sterically blocking larger proteins (e.g., streptavidin $1990(52.8 \mathrm{kDa})) .^{117}$ Importantly, a tuning of the polymer length 1991 facilitates some control over the protein size the film is 1992 pervious to.

\subsection{Simulation Assisted Sensory Design and Data} 1993 Analytics

1994 While various computational methods have been utilized to 1995 elucidate interfacial processes associated with fouling, many 1996 designs have not been analyzed computationally. With the 1997 advent of ever stronger computational methods the simulation1998 assisted design of chemically complex or chemical-physical 1999 antifouling composite-based sensing interfaces will become 2000 more routine in supporting highly specific sensor design. ${ }^{301}$ 2001 For example, in a recent study simulations were carried out on 2002 the performance of polymer coatings on nanoparticles with 2003 respect to their antifouling and cellular uptake properties. ${ }^{302}$ 2004 Analyte diffusion across fluidic channels has also been 2005 intensively scrutinized by simulations, ${ }^{303-305}$ and models 2006 developed within this could be expanded to elucidate the 2007 diffusion of analytes through porous antifouling architectures. 2008 This would then offer a more rational sensory design pathway. 2009 An additionally important consideration in the construction 2010 of sensors is the number of parameters (e.g., see Table 1) that 2011 are (ideally) optimized and monitored, particularly for in vivo 2012 applications. For any viable demonstration of scalability, the 2013 use of advanced data and statistical analyses is critical. One 2014 important parameter in almost all sensory applications is the 2015 transducing signal baseline stability; any drift here has a very 2016 direct impact on signal/noise and thus analytical sensitivity. An 2017 ability to track and mathematically correct for such drift can 2018 potentially mitigate deleterious effects. Data analysis via 2019 optimized algorithms can also identify the parameters which 2020 are most analytically useful, as demonstrated via an immittance 2021 function analysis of impedimetric data (see Figure 11). ${ }^{125}$ 2022 Though applied here to an impedance configuration, such analysis is applicable to a variety of different sensors, especially 2023 when complex signal outputs are encountered. Another data 2024 analytical tool that has received increasing attention is machine 2025 learning (ML). ML algorithms can potentially aid in in the 2026 design of improved sensory (antifouling) interfaces optimizing 2027 for desired sensory performance based on existing data 2028 sets. $^{306,307}$ These, and related, mathematical and statistical 2029 tools become increasingly important when analyzing a large 2030 number of repeat measurements (e.g., to assess reproduci- 2031 bility) or when sensing is performed in a multiplexed manner 2032 or on sensing arrays.

2033

\subsection{Highly Integrated PoC Sensing Devices}

There are many emerging technologies based on advanced 2034 micro/nanofabrication, 3-D printing, self-powered devices and 2035 soft electronic techniques with significant potential for a 2036 myriad of sensing applications. ${ }^{308,309}$ The integration of the 2037 antifouling technologies detailed herein (especially chemical/ 2038 physical strategies) could further enhance their long-term 2039 stability and application most notably for in vitro and in vivo 2040 diagnostics/theranostics. Such integrated approaches will also 2041 find home in stimuli-responsive (intelligent) biointerfaces in, 2042 for example, improved wearable sensors, ${ }^{310,311}$ organ-on-a-chip 2043 applications $^{312}$ and cell-based sensors for drug screening. ${ }^{313,314} 2044$

\section{AUTHOR INFORMATION}

2045

Corresponding Authors

Xiliang Luo - Key Laboratory of Optic-electric Sensing and 2047 Analytical Chemistry for Life Science, MOE, Shandong Key 2048 Laboratory of Biochemical Analysis, College of Chemistry and 2049 Molecular Engineering, Qingdao University of Science and 2050 Technology, Qingdao 266042, China; (1) orcid.org/0000- 2051 0001-6075-7089; Email: xiliangluo@qust.edu.cn 2052

Jason J. Davis - Department of Chemistry, University of Oxford, 2053 Oxford OX1 3QZ, United Kingdom; (1) orcid.org/0000-0001- 2054 7734-1709; Email: jason.davis@chem.ox.ac.uk 2055

Authors

2056

Cheng Jiang - Department of Chemistry and Nuffield 2057 Department of Clinical Neurosciences, University of Oxford, 2058 Oxford OX1 3QZ, United Kingdom

2059

Guixiang Wang - Key Laboratory of Optic-electric Sensing and 2060 Analytical Chemistry for Life Science, MOE, Shandong Key 2061 Laboratory of Biochemical Analysis, College of Chemistry and 2062 Molecular Engineering, Qingdao University of Science and 2063 Technology, Qingdao 266042, China; College of Chemistry and 2064 Chemical Engineering, Taishan University, Taian 271021, 2065 China

2065

Robert Hein - Department of Chemistry, University of Oxford, 2067 Oxford OX1 3QZ, United Kingdom; (1) orcid.org/0000-0001- 2068 8567-0924

Nianzu Liu - Key Laboratory of Optic-electric Sensing and 2070 Analytical Chemistry for Life Science, MOE, Shandong Key 2071 Laboratory of Biochemical Analysis, College of Chemistry and 2072 Molecular Engineering, Qingdao University of Science and 2073 Technology, Qingdao 266042, China

Complete contact information is available at:

https://pubs.acs.org/10.1021/acs.chemrev.9b00739

2074

\section{Author Contributions}

${ }^{\mathrm{I}}$ These authors contributed equally to this work. 


\section{Notes}

2080 The authors declare no competing financial interest.

\section{Biographies}

2082 Cheng Jiang received his $\mathrm{PhD}$ degree in chemistry from the University 2083 of New Souths Wales (Australia) in 2016. He was then recruited in a 2084 joint programme between Nuffield Department of Clinical Neuro2085 sciences (NDCN) and the Department of Chemistry as a 2086 postdoctoral fellow at the University of Oxford. His main research 2087 interests include the screening and validation of circulating 2088 biomarkers using high-throughput multiplexed sensing platforms 2089 towards disease diagnostics, stratification, and heath management.

2090 Guixiang Wang received a Bachelor's degree in Chemistry from 2091 Liaocheng University in 2002, a Master's degree in analytical 2092 chemistry from Liaocheng University in 2005, and a $\mathrm{PhD}$ degree in 2093 Applied Chemistry from Qingdao University of Science and 2094 Technology in 2018. She joined the Taishan University in 2005 2095 and was promoted to lecturer in 2007. She currently serves as lecturer 2096 of College of Chemistry and Chemical Engineering, Taishan 2097 University. Her scientific research interests mainly focus on 2098 conducting polymers and antifouling biosensing.

2099 Robert Hein obtained his B.Sc. from Jacobs University Bremen, 2100 Germany in 2016, where he worked in the field of supramolecular 2101 chemistry under the supervision of Prof. Werner M. Nau. During his 2102 studies, he spent a semester abroad at Cornell University (with Prof. 2103 Geoffrey W. Coates). He is currently pursuing a $\mathrm{PhD}$ at the University 2104 of Oxford under the guidance of Prof. Paul D. Beer and Prof. Jason J. 2105 Davis working on electrochemical, supramolecular anion sensors. 2106 Other research interests include the development of novel antifouling 2107 interfaces for electrochemical (bio)sensors, surface functionalization 2108 via diazonium chemistry, and host-guest chemistry.

2109 Nianzu Liu received her B.S. degree in Marine Science from Qingdao 2110 University of Science and Technology in 2016. She is currently a PhD 2111 candidate majoring in Applied Chemistry of the College of Chemistry 2112 and Molecular Engineering, Qingdao University of Science and 2113 Technology, under the supervision of Prof. Xiliang Luo. Her scientific 2114 research interests focus on biochemical analysis, nanobiosensing, and 2115 electrochemistry.

2116 Xiliang Luo received his $\mathrm{PhD}$ degree from Nanjing University in 2117 2005. He then worked as a postdoctoral fellow at Dublin City 2118 University, Arizona State University, and the University of Pittsburgh 2119 successively. In 2011, he became a research assistant professor in the 2120 Department of Bioengineering, University of Pittsburgh and a senior 2121 Marie Curie Fellow in the Department of Chemistry, University of 2122 Oxford. He then joined the Qingdao University of Science and 2123 Technology at the end of 2011 as a Taishan Scholar professor. His 2124 scientific interests are focused on biochemical analysis, electro2125 chemistry, and conducting polymers.

2126 Jason Davis is a Professor of Chemistry and a Tutorial Fellow and Dr. 2127 Lee's Reader in Chemistry at Christ Church, Oxford. He studied 2128 Chemistry at Kings College London, where he was awarded a First 2129 Class honours degree in 1993. He then undertook a DPhil in 2130 Chemistry at Oxford (1998) prior to being elected to an 2131 Extraordinary Junior Research Fellowship at The Queens College in 2132 1998, a Royal Society University Research Fellowship in 1999, and a 2133 Lectureship in Chemistry at Jesus College, Oxford, in 2001. He was 2134 made a University Lecturer and Official Student and Tutor in 2135 Chemistry at Christ Church in 2003. He was made a full professor in 21362014 . His research interests are broad and primarily focussed on the 2137 design and utilization of advanced functional interfaces, particularly those associated with diagnostics, sensing, molecular switches, and 2138 imaging.

\section{ACKNOWLEDGMENTS}

We acknowledge financial support from the National Natural 2141 Science Foundation of China (21974075, 21675093), the 2142 Taishan Scholar Program of Shandong Province of China 2143 (ts20110829), the Shandong Provincial Natural Science 2144 Foundation (ZR2019MB039), the Shandong Key Laboratory 2145 of Biochemical Analysis (SKLBA1905), and Doctoral Research 2146 Initiation Project of Taishan University (Y-01-2018018). J.J.D. 2147 wishes to acknowledge support from the EPSRC, Weston 2148 Foundation, CRUK, and the Royal Society and Osler 2149 Diagnostics. The authors would like to thank Dr. Clare F. 2150 Megarity and Dr. Amol V. Patil for their helpful comments. 2151

\section{REFERENCES}

(1) Wu, L.; Qu, X. G. Cancer Biomarker Detection: Recent 2153 Achievements and Challenges. Chem. Soc. Rev. 2015, 44, 2963-2997. 2154

(2) Sawyers, C. L. The Cancer Biomarker Problem. Nature 2008, 2155 $452,548-552$.

2156

(3) Wulfkuhle, J. D.; Liotta, L. A.; Petricoin, E. F. Proteomic 2157 Applications for the Early Detection of Cancer. Nat. Rev. Cancer 2003, 2158 3, 267.

2159

(4) Luo, X.; Davis, J. J. Electrical Biosensors and the Label Free 2160 Detection of Protein Disease Biomarkers. Chem. Soc. Rev. 2013, 42, 2161 $5944-5962$.

(5) Gooding, J. J. What Is a "Real Sample"? ACS. Sens. 2018, 3, 2163 1609-1609.

2164

(6) Barfidokht, A.; Gooding, J. J. Approaches toward Allowing 2165 Electroanalytical Devices to Be Used in Biological Fluids. Electro- 2166 analysis 2014, 26, 1182-1196.

(7) Jiang, C. Protein-resistant Electrodes for Biosensing, $\mathrm{PhD}$ thesis, 2168 University of New Souths Wales, 2016.

2169

(8) Harding, J. L.; Reynolds, M. M. Combating Medical Device 2170 Fouling. Trends Biotechnol. 2014, 32, 140-146.

2171

(9) Jaffer, I. H.; Fredenburgh, J. C.; Hirsh, J.; Weitz, J. I. Medical 2172 Device-induced Thrombosis: What Causes It and How Can We 2173 Prevent It? J. Thromb. Haemostasis 2015, 13, S72-S81. 2174

(10) Liu, B. S.; Liu, X.; Shi, S.; Huang, R. L.; Su, R. X.; Qi, W.; He, 2175 Z. M. Design and Mechanisms of Antifouling Materials for Surface 2176 Plasmon Resonance Sensors. Acta Biomater. 2016, 40, 100-118. 2177 (11) Vaisocherová, H.; Brynda, E.; Homola, J. Functionalizable Low- 2178 Fouling Coatings for Label-Free Biosensing in Complex Biological 2179 Media: Advances and Applications. Anal. Bioanal. Chem. 2015, 407, 2180 3927-3953.

2181

(12) Lowe, S.; O’Brien-Simpson, N. M.; Connal, L. A. Antibiofoul- 2182 ing Polymer Interfaces: Poly(ethylene glycol) and Other Promising 2183 Candidates. Polym. Chem. 2015, 6, 198-212.

2184

(13) Emilsson, G.; Schoch, R. L.; Feuz, L.; Höök, F.; Lim, R. Y. H.; 2185 Dahlin, A. B. Strongly Stretched Protein Resistant Poly(ethylene 2186 glycol) Brushes Prepared by Grafting-To. ACS Appl. Mater. Interfaces 2187 2015, 7, 7505-7515.

2188

(14) Akbari, A.; Yegani, R.; Pourabbas, B. Synthesis of Poly 2189 (ethylene glycol)(PEG) Grafted Silica Nanoparticles with a Minimum 2190 Adhesion of Proteins via One-Pot One-Step Method. Colloids Surf., A 2191 2015, 484, 206-215.

2192

(15) Schlenoff, J. B. Zwitteration: Coating Surfaces with Zwitterionic 2193 Functionality to Reduce Nonspecific Adsorption. Langmuir 2014, 30, 2194 9625-9636.

2195

(16) Izquierdo-Barba, I.; Colilla, M.; Vallet-Regí, M. Zwitterionic 2196 Ceramics for Biomedical Applications. Acta Biomater. 2016, 40, 201- 2197 211.

2198

(17) He, M.; Gao, K.; Zhou, L.; Jiao, Z.; Wu, M.; Cao, J.; You, X.; 2199 Cai, Z.; Su, Y.; Jiang, Z. Zwitterionic Materials for Antifouling 2200 Membrane Surface Construction. Acta Biomater. 2016, 40, 142-152. 2201 
2202 (18) Ham, H. O.; Park, S. H.; Kurutz, J. W.; Szleifer, I. G.; 2203 Messersmith, P. B. Antifouling Glycocalyx-Mimetic Peptoids. J. Am. 2204 Chem. Soc. 2013, 135, 13015-13022.

2205 (19) Leng, C.; Buss, H. G.; Segalman, R. A.; Chen, Z. Surface 2206 Structure and Hydration of Sequence-Specific Amphiphilic Poly2207 peptoids for Antifouling/Fouling Release Applications. Langmuir 2208 2015, 31, 9306-9311.

2209 (20) Sakala, G. P.; Reches, M. Peptide-Based Approaches to Fight 2210 Biofouling. Adv. Mater. Interfaces 2018, 5, 1800073.

2211 (21) Huang, R.; Liu, X.; Ye, H.; Su, R.; Qi, W.; Wang, L.; He, Z. 2212 Conjugation of Hyaluronic Acid onto Surfaces via the Interfacial 2213 Polymerization of Dopamine to Prevent Protein Adsorption. 2214 Langmuir 2015, 31, 12061-12070.

2215 (22) Zhang, C.; Liu, S. T.; Tan, L.; Zhu, H. K.; Wang, Y. M. Star2216 shaped Poly(2-methyl-2-oxazoline)-Based Films: Rapid Preparation 2217 and Effects of Polymer Architecture on Antifouling Properties. J. 2218 Mater. Chem. B 2015, 3, 5615-5628.

2219 (23) Weydert, S.; Zurcher, S.; Tanner, S.; Zhang, N.; Ritter, R.; 2220 Peter, T.; Aebersold, M. J.; Thompson-Steckel, G.; Forro, C.; 2221 Rottmar, M.; et al. Easy to Apply Polyoxazoline-Based Coating for 2222 Precise and Long-Term Control of Neural Patterns. Langmuir 2017, 2223 33, 8594-8605.

2224 (24) Wang, W.; Jayachandran, S.; Li, M.; Xu, S.; Luo, X. Hyaluronic 2225 Acid Functionalized Nanostructured Sensing Interface for Voltam2226 metric Determination of microRNA in Biological Media with Ultra2227 High Sensitivity and Ultra-Low Fouling. Microchim. Acta 2018, 185, 2228156.

2229 (25) Caracciolo, G.; Farokhzad, O. C.; Mahmoudi, M. Biological 2230 Identity of Nanoparticles in Vivo: Clinical Implications of the Protein 2231 Corona. Trends Biotechnol. 2017, 35, 257-264.

2232 (26) Bertrand, N.; Grenier, P.; Mahmoudi, M.; Lima, E. M.; Appel, 2233 E. A.; Dormont, F.; Lim, J.-M.; Karnik, R.; Langer, R.; Farokhzad, O. 2234 C. Mechanistic Understanding of in Vivo Protein Corona Formation 2235 on Polymeric Nanoparticles and Impact on Pharmacokinetics. Nat. 2236 Commun. 2017, 8, 777.

2237 (27) Chen, S.; Cao, Z.; Jiang, S. Ultra-Low Fouling Peptide Surfaces 2238 Derived from Natural Amino Acids. Biomaterials 2009, 30, 589222395896.

2240 (28) Blackman, L. D.; Gunatillake, P. A.; Cass, P.; Locock, K. E. S. 2241 An Introduction to Zwitterionic Polymer Behavior and Applications 2242 in Solution and at Surfaces. Chem. Soc. Rev. 2019, 48, 757-770.

2243 (29) Baggerman, J.; Smulders, M. M. J.; Zuilhof, H. Romantic 2244 Surfaces: A Systematic Overview of Stable, Biospecific, and 2245 Antifouling Zwitterionic Surfaces. Langmuir 2019, 35, 1072-1084.

2246 (30) Hakobyan, S.; Rzhepishevska, O.; Barbero, D. R.; Ramstedt, M. 2247 Functionalization of Zwitterionic Polymer Brushes, Do They Remain 2248 Antifouling? Surf. Interface Anal. 2018, 50, 1001-1006.

2249 (31) Leckband, D.; Sheth, S.; Halperin, A. Grafted Poly (ethylene 2250 oxide) Brushes as Nonfouling Surface Coatings. J. Biomater. Sci., 2251 Polym. Ed. 1999, 10, 1125-1147.

2252 (32) Kruis, I. C.; Lowik, D.; Boelens, W. C.; van Hest, J. C. M.; 2253 Pruijn, G. J. M. An Integrated, Peptide-Based Approach to Site2254 Specific Protein Immobilization for Detection of Biomolecular 2255 Interactions. Analyst 2016, 141, 5321-5328.

2256 (33) Cui, M.; Wang, Y.; Wang, H.; Wu, Y.; Luo, X. A Label-Free 2257 Electrochemical DNA Biosensor for Breast Cancer Marker BRCA1 2258 Based on Self-Assembled Antifouling Peptide Monolayer. Sens. 2259 Actuators, B 2017, 244, 742-749.

2260 (34) Nowinski, A. K.; Sun, F.; White, A. D.; Keefe, A. J.; Jiang, S. 2261 Sequence, Structure, and Function of Peptide Self-Assembled 2262 Monolayers. J. Am. Chem. Soc. 2012, 134, 6000-6005.

2263 (35) Kuo, T. M.; Shen, M. Y.; Huang, S. Y.; Li, Y. K.; Chuang, M. C. 2264 Facile Fabrication of a Sensor with a Bifunctional Interface for Logic 2265 Analysis of the New Delhi Metallo- $\beta$-Lactamase (NDM)-Coding 2266 Gene. ACS. Sens. 2016, 1, 124-130.

2267 (36) Jiang, C.; Alam, M. T.; Parker, S. G.; Darwish, N.; Gooding, J. J. 2268 Strategies To Achieve Control over the Surface Ratio of Two 2269 Different Components on Modified Electrodes Using Aryldiazonium 2270 Salts. Langmuir 2016, 32, 2509-2517.
(37) Wang, J.; Hui, N. A Nonfouling Voltammetric Immunosensor 2271 for the Carcinoembryonic Antigen based on the Use of Polyaniline 2272 Nanowires Wrapped with Hyaluronic Acid. Microchim. Acta 2018, 2273 $185,329$. 2274

(38) Hui, N.; Sun, X. T.; Niu, S. Y.; Luo, X. L. PEGylated 2275 Polyaniline Nanofibers: Antifouling and Conducting Biomaterial for 2276 Electrochemical DNA Sensing. ACS Appl. Mater. Interfaces 2017, 9, 2277 2914-2923.

2278

(39) Vaisocherova-Lisalova, H.; Visova, I.; Ermini, M. L.; Springer, 2279 T.; Song, X. C.; Mrazek, J.; Lamacova, J.; Lynn, N. S.; Sedivak, P.; 2280 Homola, J. Low-Fouling Surface Plasmon Resonance Biosensor for 2281 Multi-Step Detection of Foodborne Bacterial Pathogens in Complex 2282 Food Samples. Biosens. Bioelectron. 2016, 80, 84-90. 2283

(40) Vaisocherova-Lisalova, H.; Surman, F.; Visova, I.; Vala, M.; 2284 Springer, T.; Ermini, M. L.; Sipova, H.; Sedivak, P.; Houska, M.; 2285 Riedel, T.; et al. Copolymer Brush-Based Ultralow-Fouling Bio- 2286 recognition Surface Platform for Food Safety. Anal. Chem. 2016, 88, 2287 10533-10539.

2288

(41) Vaisocherova, H.; Sipova, H.; Visova, I.; Bockova, M.; Springer, 2289 T.; Ermini, M. L.; Song, X.; Krejcik, Z.; Chrastinova, L.; Pastva, O.; 2290 et al. Rapid and Sensitive Detection of Multiple MicroRNAs in Cell 2291 Lysate by Low-fouling Surface Plasmon Resonance Biosensor. Biosens. 2292 Bioelectron. 2015, 70, 226-231.

2293

(42) Riedel, T.; Surman, F.; Hageneder, S.; Pop-Georgievski, O.; 2294 Noehammer, C.; Hofner, M.; Brynda, E.; Rodriguez-Emmenegger, C.; 2295 Dostalek, J. Hepatitis B Plasmonic Biosensor for the Analysis of 2296 Clinical Serum Samples. Biosens. Bioelectron. 2016, 85, 272-279. 2297

(43) Riedel, T.; Hageneder, S.; Surman, F.; Pop-Georgievski, O.; 2298 Noehammer, C.; Hofner, M.; Brynda, E.; Rodriguez-Emmenegger, C.; 2299 Dostálek, J. Plasmonic Hepatitis B Biosensor for the Analysis of 2300 Clinical Saliva. Anal. Chem. 2017, 89, 2972-2977.

2301

(44) Shen, W.; Chang, Y.; Liu, G.; Wang, H.; Cao, A.; An, Z. 2302 Biocompatible, Antifouling, and Thermosensitive Core-Shell Nano- 2303 gels Synthesized by RAFT Aqueous Dispersion Polymerization. 2304 Macromolecules 2011, 44, 2524-2530.

2305

(45) Kitano, H.; Kondo, T.; Kamada, T.; Iwanaga, S.; Nakamura, 2306 M.; Ohno, K. Anti-biofouling Properties of an Amphoteric Polymer 2307 Brush Constructed on a Glass Substrate. Colloids Surf., B 2011, 88, 2308 455-462.

2309

(46) Lin, P.; Chuang, T.-L.; Chen, P. Z.; Lin, C.-W.; Gu, F. X. Low- 2310 Fouling Characteristics of Ultrathin Zwitterionic Cysteine SAMs. 2311 Langmuir 2019, 35, 1756-1767.

2312

(47) Bryan, T.; Luo, X.; Forsgren, L.; Morozova-Roche, L. A.; Davis, 2313 J. J. The Robust Electrochemical Detection of a Parkinson's Disease 2314 Marker in Whole Blood Sera. Chem. Sci. 2012, 3, 3468-3473. 2315

(48) Li, Q.; Imbrogno, J.; Belfort, G.; Wang, X.-L. Making Polymeric 2316 Membranes Antifouling via "Grafting from" Polymerization of 2317 Zwitterions. J. Appl. Polym. Sci. 2015, 132, 41781.

(49) Sin, M.-C.; Chen, S.-H.; Chang, Y. Hemocompatibility of 2319 Zwitterionic Interfaces and Membranes. Polym. J. 2014, 46, 436. 2320

(50) Heggestad, J. T.; Fontes, C. M.; Joh, D. Y.; Hucknall, A. M.; 2321 Chilkoti, A. In Pursuit of Zero 2.0: Recent Developments in 2322 Nonfouling Polymer Brushes for Immunoassays. Adv. Mater. 2020, 2323 32, 1903285.

2324

(51) Cao, C.; Zhang, Y.; Jiang, C.; Qi, M.; Liu, G. Advances on 2325 Aryldiazonium Salt Chemistry Based Interfacial Fabrication for 2326 Sensing Applications. ACS Appl. Mater. Interfaces 2017, 9, 5031- 2327 5049.

2328

(52) Pinson, J.; Podvorica, F. Attachment of Organic Layers to 2329 Conductive or Semiconductive Surfaces by Reduction of Diazonium 2330 Salts. Chem. Soc. Rev. 2005, 34, 429-439.

2331

(53) Liu, G.; Böcking, T.; Gooding, J. J. Diazonium salts: Stable 2332 Monolayers on Gold Electrodes for Sensing Applications. J. 2333 Electroanal. Chem. 2007, 600, 335-344.

2334

(54) Jiang, C.; Alam, M. T.; Silva, S. M.; Taufik, S.; Fan, S.; 2335 Gooding, J. J. Unique Sensing Interface That Allows the Development 2336 of an Electrochemical Immunosensor for the Detection of Tumor 2337 Necrosis Factor $\alpha$ in Whole Blood. ACS. Sens. 2016, 1, 1432-1438. 2338 
2339 (55) Khor, S. M.; Liu, G.; Fairman, C.; Iyengar, S. G.; Gooding, J. J. 2340 The Importance of Interfacial Design for the Sensitivity of a Label-free 2341 Electrochemical Immuno-Biosensor for Small Organic Molecules. 2342 Biosens. Bioelectron. 2011, 26, 2038-2044.

2343 (56) Jiang, C.; Silva, S. M.; Fan, S.; Wu, Y.; Alam, M. T.; Liu, G.; 2344 Gooding, J. J. Aryldiazonium Salt Derived Mixed Organic Layers: 2345 From Surface Chemistry to their Applications. J. Electroanal. Chem. 2346 2017, 785, 265-278.

2347 (57) Jiang, C.; Tanzirul Alam, M.; Parker, S. G.; Gooding, J. J. 2348 Zwitterionic Phenyl Phosphorylcholine on Indium Tin Oxide: a Low2349 Impedance Protein-Resistant Platform for Biosensing. Electroanalysis 2350 2015, 27, 884-889.

2351 (58) Chen, S.; Li, L.; Zhao, C.; Zheng, J. Surface Hydration: 2352 Principles and Applications toward Low-Fouling/Nonfouling Bio2353 materials. Polymer 2010, 51, 5283-5293.

2354 (59) Zhang, K.; Huang, H.; Hung, H.-C.; Leng, C.; Wei, S.; Crisci, 2355 R.; Jiang, S.; Chen, Z. Strong Hydration at the Poly(ethylene glycol) 2356 Brush/Albumin Solution Interface. Langmuir 2020, 36, 2030-2036. 2357 (60) Chen, S.; Zheng, J.; Li, L.; Jiang, S. Strong Resistance of 2358 Phosphorylcholine Self-Assembled Monolayers to Protein Adsorp2359 tion: Insights into Nonfouling Properties of Zwitterionic Materials. J. $2360 \mathrm{Am}$. Chem. Soc. 2005, 127, 14473-14478.

2361 (61) Shao, Q.; Jiang, S. Molecular Understanding and Design of 2362 Zwitterionic Materials. Adv. Mater. 2015, 27, 15-26.

2363 (62) Zhang, Y.; Liu, Y.; Ren, B.; Zhang, D.; Xie, S.; Chang, Y.; Yang, 2364 J.; Wu, J.; Xu, L.; Zheng, J. Fundamentals and Applications of 2365 Zwitterionic Antifouling Polymers. J. Phys. D: Appl. Phys. 2019, 52, 2366403001.

2367 (63) Wu, J.; Lin, W.; Wang, Z.; Chen, S.; Chang, Y. Investigation of 2368 the Hydration of Nonfouling Material Poly(sulfobetaine methacry2369 late) by Low-Field Nuclear Magnetic Resonance. Langmuir 2012, 28, $23707436-7441$

2371 (64) Wu, J.; Chen, S. Investigation of the Hydration of Nonfouling 2372 Material Poly(ethylene glycol) by Low-Field Nuclear Magnetic 2373 Resonance. Langmuir 2012, 28, 2137-2144.

2374 (65) Baumgartner, S.; Lahajnar, G.; Sepe, A.; Kristl, J. Quantitative 2375 Evaluation of Polymer Concentration Profile during Swelling of 2376 Hydrophilic Matrix Tablets Using $1 \mathrm{H}$ NMR and MRI Methods. Eur. 2377 J. Pharm. Biopharm. 2005, 59, 299-306.

2378 (66) Perry, A.; Neipert, C.; Space, B.; Moore, P. B. Theoretical 2379 Modeling of Interface Specific Vibrational Spectroscopy: Methods 2380 and Applications to Aqueous Interfaces. Chem. Rev. 2006, 106, 123423811258.

2382 (67) Nagasawa, D.; Azuma, T.; Noguchi, H.; Uosaki, K.; Takai, M. 2383 Role of Interfacial Water in Protein Adsorption onto Polymer Brushes 2384 as Studied by SFG Spectroscopy and QCM. J. Phys. Chem. C 2015, $2385119,17193-17201$.

2386 (68) Wang, H.-F. Sum Frequency Generation Vibrational Spectros2387 copy (SFG-VS) for Complex Molecular Surfaces and Interfaces: 2388 Spectral Lineshape Measurement and Analysis Plus Some Con2389 troversial Issues. Prog. Surf. Sci. 2016, 91, 155-182.

2390 (69) Leng, C.; Hung, H.-C.; Sieggreen, O. A.; Li, Y.; Jiang, S.; Chen, 2391 Z. Probing the Surface Hydration of Nonfouling Zwitterionic and 2392 Poly(ethylene glycol) Materials with Isotopic Dilution Spectroscopy. 2393 J. Phys. Chem. C 2015, 119, 8775-8780.

2394 (70) Leng, C.; Hung, H.-C.; Sun, S.; Wang, D.; Li, Y.; Jiang, S.; 2395 Chen, Z. Probing the Surface Hydration of Nonfouling Zwitterionic 2396 and PEG Materials in Contact with Proteins. ACS Appl. Mater. 2397 Interfaces 2015, 7, 16881-16888.

2398 (71) Hankett, J. M.; Liu, Y.; Zhang, X.; Zhang, C.; Chen, Z. 2399 Molecular Level Studies of Polymer Behaviors at the Water Interface 2400 Using Sum Frequency Generation Vibrational Spectroscopy. J. Polym. 2401 Sci., Part B: Polym. Phys. 2013, 51, 311-328.

2402 (72) Leng, C.; Sun, S.; Zhang, K.; Jiang, S.; Chen, Z. Molecular 2403 Level Studies on Interfacial Hydration of Zwitterionic and Other 2404 Antifouling Polymers in Situ. Acta Biomater. 2016, 40, 6-15.

2405 (73) Leung, B. O.; Yang, Z.; Wu, S. S. H.; Chou, K. C. Role of 2406 Interfacial Water on Protein Adsorption at Cross-Linked Polyethylene 2407 Oxide Interfaces. Langmuir 2012, 28, 5724-5728.
(74) Ziemba, C.; Khavkin, M.; Priftis, D.; Acar, H.; Mao, J.; Benami, 2408 M.; Gottlieb, M.; Tirrell, M.; Kaufman, Y.; Herzberg, M. Antifouling 2409 Properties of a Self-Assembling Glutamic Acid-Lysine Zwitterionic 2410 Polymer Surface Coating. Langmuir 2019, 35, 1699-1713. 2411

(75) Wang, R. Y.; Himmelhaus, M.; Fick, J.; Herrwerth, S.; Eck, W.; 2412 Grunze, M. Interaction of Self-Assembled Monolayers of Oligo- 2413 (ethylene glycol)-Terminated Alkanethiols with Water Studied by 2414 Vibrational Sum-Frequency Generation. J. Chem. Phys. 2005, 122, 2415 164702 .

2416

(76) Han, X.; Leng, C.; Shao, Q.; Jiang, S.; Chen, Z. Absolute 2417 Orientations of Water Molecules at Zwitterionic Polymer Interfaces 2418 and Interfacial Dynamics after Salt Exposure. Langmuir 2019, 35, 2419 $1327-1334$.

2420

(77) Zhang, C.; Parada, G. A.; Zhao, X.; Chen, Z. Probing Surface 2421 Hydration and Molecular Structure of Zwitterionic and Polyacryla- 2422 mide Hydrogels. Langmuir 2019, 35, 13292-13300. 2423

(78) Leng, C.; Huang, H.; Zhang, K.; Hung, H.-C.; Xu, Y.; Li, Y.; 2424 Jiang, S.; Chen, Z. Effect of Surface Hydration on Antifouling 2425 Properties of Mixed Charged Polymers. Langmuir 2018, 34, 6538- 2426 6545.

2427

(79) Jeon, S. I.; Lee, J. H.; Andrade, J. D.; De Gennes, P. G. 2428 Protein-Surface Interactions in the Presence of Polyethylene Oxide: 2429 I. Simplified Theory. J. Colloid Interface Sci. 1991, 142, 149-158. 2430

(80) Zheng, J.; Li, L.; Tsao, H.-K.; Sheng, Y.-J.; Chen, S.; Jiang, S. 2431 Strong Repulsive Forces between Protein and Oligo (ethylene glycol) 2432 Self-Assembled Monolayers: A Molecular Simulation Study. Biophys. 2433 J. 2005, 89, 158-166. 2434

(81) He, Y.; Hower, J.; Chen, S.; Bernards, M. T.; Chang, Y.; Jiang, 2435 S. Molecular Simulation Studies of Protein Interactions with 2436 Zwitterionic Phosphorylcholine Self-Assembled Monolayers in the 2437 Presence of Water. Langmuir 2008, 24, 10358-10364. 2438

(82) Shao, Q.; He, Y.; White, A. D.; Jiang, S. Difference in 2439 Hydration between Carboxybetaine and Sulfobetaine. J. Phys. Chem. B 2440 2010, 114, 16625-16631.

(83) Shao, Q.; Mi, L.; Han, X.; Bai, T.; Liu, S.; Li, Y.; Jiang, S. 2442 Differences in Cationic and Anionic Charge Densities Dictate 2443 Zwitterionic Associations and Stimuli Responses. J. Phys. Chem. B 2444 2014, 118, 6956-6962.

2445

(84) Xie, Y.; Pan, Y.; Zhang, R.; Liang, Y.; Li, Z. Modulating Protein 2446 Behaviors on Responsive Surface by External Electric Fields: A 2447 Molecular Dynamics Study. Appl. Surf. Sci. 2015, 326, 55-65. 2448

(85) Rabe, M.; Verdes, D.; Seeger, S. Understanding Protein 2449 Adsorption Phenomena at Solid Surfaces. Adv. Colloid Interface Sci. 2450 2011, 162, 87-106.

2451

(86) Zhang, Z.; Zhang, M.; Chen, S.; Horbett, T. A.; Ratner, B. D.; 2452 Jiang, S. Blood Compatibility of Surfaces with Superlow Protein 2453 Adsorption. Biomaterials 2008, 29, 4285-4291.

2454

(87) Sun, F.; Hung, H.-C.; Sinclair, A.; Zhang, P.; Bai, T.; Galvan, D. 2455 D.; Jain, P.; Li, B.; Jiang, S.; Yu, Q. Hierarchical Zwitterionic 2456 Modification of A SERS Substrate Enables Real-Time Drug 2457 Monitoring in Blood Plasma. Nat. Commun. 2016, 7, 13437.

(88) Gui, A. L.; Luais, E.; Peterson, J. R.; Gooding, J. J. Zwitterionic 2459 Phenyl Layers: Finally, Stable, Anti-Biofouling Coatings That Do Not 2460 Passivate Electrodes. ACS Appl. Mater. Interfaces 2013, 5, 4827-4835. 2461

(89) Darwish, N. T.; Alias, Y.; Khor, S. M. Indium Tin Oxide with 2462 Zwitterionic Interfacial Design for Biosensing Applications in 2463 Complex Matrices. Appl. Surf. Sci. 2015, 325, 91-99.

2464

(90) Taufik, S.; Barfidokht, A.; Alam, M. T.; Jiang, C.; Parker, S. G.; 2465 Gooding, J. J. An Antifouling Electrode Based on Electrode-Organic 2466 Layer-Nanoparticle Constructs: Electrodeposited Organic Layers 2467 Versus Self-Assembled Monolayers. J. Electroanal. Chem. 2016, 779, 2468 229-235.

2469

(91) Parviz, M.; Darwish, N.; Alam, M. T.; Parker, S. G.; Ciampi, S.; 2470 Gooding, J. J. Investigation of the Antifouling Properties of Phenyl 2471 Phosphorylcholine-Based Modified Gold Surfaces. Electroanalysis 2472 2014, 26, 1471-1480.

(92) Zhang, Y. X.; Islam, N.; Carbonell, R. G.; Rojas, O. J. Specific 2474 Binding of Immunoglobulin G with Bioactive Short Peptides 2475 Supported on Antifouling Copolymer Layers for Detection in Quartz 2476 
2477 Crystal Microgravimetry and Surface Plasmon Resonance. Anal. 2478 Chem. 2013, 85, 1106-1113.

2479 (93) Cao, B.; Lee, C. J.; Zeng, Z. P.; Cheng, F.; Xu, F. J.; Cong, H. 2480 B.; Cheng, G. Electroactive poly(sulfobetaine-3,4-ethylenedioxythio2481 phene) (PSBEDOT) with Controllable Antifouling and Antimicrobial 2482 Properties. Chem. Sci. 2016, 7, 1976-1981.

2483 (94) Huang, C.-J.; Wang, L.-C.; Liu, C.-Y.; Chiang, A. S.; Chang, Y.2484 C. Natural Zwitterionic Organosulfurs as Surface Ligands for 2485 Antifouling and Responsive Properties. Biointerphases 2014, 9, 2486 No. 029010.

2487 (95) Luan, Y.; Li, D.; Wei, T.; Wang, M.; Tang, Z.; Brash, J. L.; 2488 Chen, H. Hearing Loss" in QCM Measurement of Protein Adsorption 2489 to Protein Resistant Polymer Brush Layers. Anal. Chem. 2017, 89, 2490 4184-4191.

2491 (96) Ogi, H.; Fukunishi, Y.; Nagai, H.; Okamoto, K.; Hirao, M.; 2492 Nishiyama, M. Nonspecific-Adsorption Behavior of Polyethylenglycol 2493 and Bovine Serum Albumin Studied by 55-MHz Wireless-Electro2494 deless Quartz Crystal Microbalance. Biosens. Bioelectron. 2009, 24, $24953148-3152$.

2496 (97) Jin, J.; Han, Y.; Zhang, C.; Liu, J.; Jiang, W.; Yin, J.; Liang, H. 2497 Effect of Grafted PEG Chain Conformation on Albumin and 2498 Lysozyme Adsorption: A Combined Study Using QCM-D and DPI. 2499 Colloids Surf., B 2015, 136, 838-844.

2500 (98) Li, B.-R.; Shen, M.-Y.; Yu, H.-h.; Li, Y.-K. Rapid Construction 2501 of An Effective Antifouling Layer on a Au Surface via Electro2502 deposition. Chem. Commun. 2014, 50, 6793-6796.

2503 (99) Cui, J.; Ju, Y.; Liang, K.; Ejima, H.; Lörcher, S.; Gause, K. T.; 2504 Richardson, J. J.; Caruso, F. Nanoscale Engineering of Low-Fouling 2505 Surfaces through Polydopamine Immobilisation of Zwitterionic 2506 Peptides. Soft Matter 2014, 10, 2656-2663.

2507 (100) Bahadır, E. B.; Sezgintürk, M. K. A Review on Impedimetric 2508 Biosensors. Artif. Cells, Nanomed., Biotechnol. 2016, 44, 248-262.

2509 (101) Xu, Q.; Cheng, H.; Lehr, J.; Patil, A. V.; Davis, J. J. Graphene 2510 Oxide Interfaces in Serum Based Autoantibody Quantification. Anal. 2511 Chem. 2015, 87, 346-350.

2512 (102) Luo, X.; Xu, M.; Freeman, C.; James, T.; Davis, J. J. 2513 Ultrasensitive Label Free Electrical Detection of Insulin in Neat Blood 2514 Serum. Anal. Chem. 2013, 85, 4129-4134.

2515 (103) Piccoli, J.; Hein, R.; El-Sagheer, A. H.; Brown, T.; Cilli, E. M.; 2516 Bueno, P. R.; Davis, J. J. Redox Capacitive Assaying of C-Reactive 2517 Protein at a Peptide Supported Aptamer Interface. Anal. Chem. 2018, 2518 90, 3005-3008.

2519 (104) Wang, G.; Xu, Q.; Liu, L.; Su, X.; Lin, J.; Xu, G.; Luo, X. 2520 Mixed Self-Assembly of Polyethylene Glycol and Aptamer on 2521 Polydopamine Surface for Highly Sensitive and Low-Fouling 2522 Detection of Adenosine Triphosphate in Complex Media. ACS 2523 Appl. Mater. Interfaces 2017, 9, 31153-31160.

2524 (105) Wang, G.; Su, X.; Xu, Q.; Xu, G.; Lin, J.; Luo, X. Antifouling 2525 Aptasensor for the Detection of Adenosine Triphosphate in Biological 2526 Media Based on Mixed Self-Assembled Aptamer and Zwitterionic 2527 Peptide. Biosens. Bioelectron. 2018, 101, 129-134.

2528 (106) Howes, P. D.; Chandrawati, R.; Stevens, M. M. Colloidal 2529 Nanoparticles as Advanced Biological Sensors. Science 2014, 346, 25301247390.

2531 (107) Wang, H.; Cheng, F.; Shen, W.; Cheng, G.; Zhao, J.; Peng, 2532 W.; Qu, J. Amino Acid-Based Anti-fouling Functionalization of Silica 2533 Nanoparticles Using Divinyl Sulfone. Acta Biomater. 2016, 40, 2732534281.

2535 (108) Sanchez-Salcedo, S.; Vallet-Regí, M.; Shanin, S. A.; Glackin, C. 2536 A.; Zink, J. I. Mesoporous Core-shell Silica Nanoparticles with Anti2537 fouling Properties for Ovarian Cancer Therapy. Chem. Eng. J. 2018, 2538 340, 114-124.

2539 (109) Affonso de Oliveira, J. F.; Scheffer, F. R.; Landis, R. F.; 2540 Teixeira Neto, É.; Rotello, V. M.; Cardoso, M. B. Dual 2541 Functionalization of Nanoparticles for Generating Corona-Free and 2542 Noncytotoxic Silica Nanoparticles. ACS Appl. Mater. Interfaces 2018, 2543 10, 41917-41923.
(110) Cheng, F.; Zhu, C.; He, W.; Zhao, J.; Qu, J. pSBMA- 2544 Conjugated Magnetic Nanoparticles for Selective IgG Separation. 2545 Langmuir 2019, 35, 1111-1118.

2546

(111) Kang, S.; Lee, M.; Kang, M.; Noh, M.; Jeon, J.; Lee, Y.; Seo, J.- 2547 H. Development of Anti-biofouling Interface on Hydroxyapatite 2548 Surface by Coating Zwitterionic MPC Polymer Containing Calcium- 2549 Binding Moieties to Prevent Oral Bacterial Adhesion. Acta Biomater. 2550 2016, 40, 70-77.

2551

(112) Estupiñán, D.; Bannwarth, M. B.; Mylon, S. E.; Landfester, K.; 2552 Muñoz-Espí, R.; Crespy, D. Multifunctional Clickable and Protein- 2553 Repellent Magnetic Silica Nanoparticles. Nanoscale 2016, 8, 3019- 2554 3030. 2555

(113) Hu, F.; Chen, K.; Xu, H.; Gu, H. Design and Preparation of 2556 Bi-Functionalized Short-Chain Modified Zwitterionic Nanoparticles. 2557 Acta Biomater. 2018, 72, 239-247. 2558

(114) Rodriguez-Emmenegger, C.; Hasan, E.; Pop-Georgievski, O.; 2559 Houska, M.; Brynda, E.; Alles, A. B. Controlled/Living Surface- 2560 Initiated ATRP of Antifouling Polymer Brushes from Gold in PBS 2561 and Blood Sera as a Model Study for Polymer Modifications in 2562 Complex Biological Media. Macromol. Biosci. 2012, 12, 525-532. 2563

(115) Hui, N.; Sun, X.; Song, Z.; Niu, S.; Luo, X. Gold 2564 Nanoparticles and Polyethylene Glycols Functionalized Conducting 2565 Polyaniline Nanowires for Ultrasensitive and Low fouling Immuno- 2566 sensing of Alpha-Fetoprotein. Biosens. Bioelectron. 2016, 86, 143-149. 2567 (116) Chen, C.-H.; Luo, S.-C. Tuning Surface Charge and 2568 Morphology for the Efficient Detection of Dopamine under the 2569 Interferences of Uric Acid, Ascorbic Acid, and Protein Adsorption. 2570 ACS Appl. Mater. Interfaces 2015, 7, 21931-21938. 2571

(117) Paloni, J. M.; Dong, X.-H.; Olsen, B. D. Protein-Polymer 2572 Block Copolymer Thin Films for Highly Sensitive Detection of Small 2573 Proteins in Biological Fluids. ACS. Sens. 2019, 4, 2869-2878. 2574

(118) Liu, D.; Xie, Y.; Shao, H.; Jiang, X. Using Azobenzene- 2575 Embedded Self-Assembled Monolayers To Photochemically Control 2576 Cell Adhesion Reversibly. Angew. Chem., Int. Ed. 2009, 48, 4406- 2577 4408.

2578

(119) Ng, C. C. A.; Magenau, A.; Ngalim, S. H.; Ciampi, S.; 2579 Chockalingham, M.; Harper, J. B.; Gaus, K.; Gooding, J. J. Using an 2580 Electrical Potential to Reversibly Switch Surfaces between Two States 2581 for Dynamically Controlling Cell Adhesion. Angew. Chem. 2012, 124, 2582 7826-7830. 2583

(120) Demirci, S.; Kinali-Demirci, S.; Jiang, S. A Switchable Polymer 2584 Brush System for Antifouling and Controlled Detection. Chem. 2585 Commun. 2017, 53, 3713-3716.

2586

(121) Ostuni, E.; Chapman, R. G.; Holmlin, R. E.; Shuichi 2587 Takayama, A.; Whitesides, G. M. A Survey of Structure-Property 2588 Relationships of Surfaces that Resist the Adsorption of Protein. 2589 Langmuir 2001, 17, 5605-5620.

2590

(122) Castiello, F. R.; Tabrizian, M. Multiplex Surface Plasmon 2591 Resonance Imaging-Based Biosensor for Human Pancreatic Islets 2592 Hormones Quantification. Anal. Chem. 2018, 90, 3132-3139. 2593 (123) Xu, M.; Luo, X.; Davis, J. J. The Label Free Picomolar 2594 Detection of Insulin in Blood Serum. Biosens. Bioelectron. 2013, 39, 2595 $21-25$.

2596

(124) Xu, Q.; Evetts, S.; Hu, M.; Talbot, K.; Wade-Martins, R.; 2597 Davis, J. J. An Impedimetric Assay of $\alpha$-Synuclein Autoantibodies in 2598 Early Stage Parkinson's Disease. RSC Adv. 2014, 4, 58773-58777. 2599

(125) Patil, A. V.; Bedatty Fernandes, F. C.; Bueno, P. R; Davis, J. J. 2600 Immittance Electroanalysis in Diagnostics. Anal. Chem. 2015, 87, 2601 944-950.

2602

(126) Bryan, T.; Luo, X.; Bueno, P. R.; Davis, J. J. An Optimised 2603 Electrochemical Biosensor for the Label-Free Detection of C-Reactive 2604 Protein in Blood. Biosens. Bioelectron. 2013, 39, 94-98. 2605

(127) Johnson, A.; Song, Q.; Ko Ferrigno, P.; Bueno, P. R.; Davis, J. 2606 J. Sensitive Affimer and Antibody Based Impedimetric Label-Free 2607 Assays for C-Reactive Protein. Anal. Chem. 2012, 84, 6553-6560. 2608 (128) Bedatty Fernandes, F. C.; Patil, A. V.; Bueno, P. R.; Davis, J. J. 2609 Optimized Diagnostic Assays Based on Redox Tagged Bioreceptive 2610 Interfaces. Anal. Chem. 2015, 87, 12137-12144. 
2612 (129) Li, Q.; Tofaris, G. K.; Davis, J. J. Concentration-Normalized 2613 Electroanalytical Assaying of Exosomal Markers. Anal. Chem. 2017, 2614 89, 3184-3190.

2615 (130) Santos, A.; Bueno, P. R.; Davis, J. J. A Dual Marker Label Free 2616 Electrochemical Assay for Flavivirus Dengue Diagnosis. Biosens. 2617 Bioelectron. 2018, 100, 519-525.

2618 (131) Luo, X.; Xu, Q.; James, T.; Davis, J. J. Redox and Label-Free 2619 Array Detection of Protein Markers in Human Serum. Anal. Chem. 2620 2014, 86, 5553-5558.

2621 (132) Liu, G.; Khor, S. M.; Iyengar, S. G.; Gooding, J. J. 2622 Development of an Electrochemical Immunosensor for the Detection 2623 of HbA1c in Serum. Analyst 2012, 137, 829-832.

2624 (133) Kaur, J.; Jiang, C.; Liu, G. Different Strategies for Detection of $2625 \mathrm{HbA1c}$ Emphasizing on Biosensors and Point-of-Care Analyzers. 2626 Biosens. Bioelectron. 2019, 123, 85-100.

2627 (134) Wang, W.; Fan, X.; Xu, S.; Davis, J. J.; Luo, X. Low Fouling 2628 Label-Free DNA Sensor Based on Polyethylene Glycols Decorated 2629 with Gold Nanoparticles for the Detection of Breast Cancer 2630 Biomarkers. Biosens. Bioelectron. 2015, 71, 51-56.

2631 (135) Chen, L.; Liu, X.; Chen, C. Impedimetric Biosensor Modified 2632 with Hydrophilic Material of Tannic Acid/Polyethylene Glycol and 2633 Dopamine-Assisted Deposition for Detection of Breast Cancer2634 Related BRCA1 Gene. J. Electroanal. Chem. 2017, 791, 204-210.

2635 (136) Chen, L.; Lv, S.; Liu, M.; Chen, C.; Sheng, J.; Luo, X. Low2636 Fouling Magnetic Nanoparticles and Evaluation of Their Potential 2637 Application as Disease Markers Assay in Whole Serum. ACS. Appl. 2638 Nano. Mater. 2018, 1, 2489-2495.

2639 (137) Rana, S.; Le, N. D. B.; Mout, R.; Duncan, B.; Elci, S. G.; Saha, 2640 K.; Rotello, V. M. A Multichannel Biosensor for Rapid Determination 2641 of Cell Surface Glycomic Signatures. ACS Cent. Sci. 2015, 1, 191-197. 2642 (138) Geng, Y.; Peveler, W. J.; Rotello, V. M. Array-Based "Chemical 2643 Nose” Sensing in Diagnostics and Drug Discovery. Angew. Chem., Int. 2644 Ed. 2019, 58, 5190-5200.

2645 (139) De, M.; Rana, S.; Akpinar, H.; Miranda, O. R.; Arvizo, R. R.; 2646 Bunz, U. H. F.; Rotello, V. M. Sensing of Proteins in Human Serum 2647 Using Conjugates of Nanoparticles and Green Fluorescent Protein. 2648 Nat. Chem. 2009, 1, 461-465.

2649 (140) Miranda, O. R.; Li, X.; Garcia-Gonzalez, L.; Zhu, Z.-J.; Yan, 2650 B.; Bunz, U. H. F.; Rotello, V. M. Colorimetric Bacteria Sensing Using 2651 a Supramolecular Enzyme-Nanoparticle Biosensor. J. Am. Chem. Soc. $26522011,133,9650-9653$.

2653 (141) Rana, S.; Le, N. D. B.; Mout, R.; Saha, K.; Tonga, G. Y.; Bain, 2654 R. E. S.; Miranda, O. R.; Rotello, C. M.; Rotello, V. M. A 2655 Multichannel Nanosensor for Instantaneous Readout of Cancer 2656 Drug Mechanisms. Nat. Nanotechnol. 2015, 10, 65.

2657 (142) Chuah, K.; Wu, Y.; Vivekchand, S. R. C.; Gaus, K.; Reece, P. 2658 J.; Micolich, A. P.; Gooding, J. J. Nanopore Blockade Sensors for 2659 Ultrasensitive Detection of Proteins in Complex Biological Samples. 2660 Nat. Commun. 2019, 10, 2109.

2661 (143) Wu, Y.; Bennett, D.; Tilley, R. D.; Gooding, J. J. How 2662 Nanoparticles Transform Single Molecule Measurements into 2663 Quantitative Sensors. Adv. Mater. 2019, 0, 1904339.

2664 (144) Wu, Y.; Tilley, R. D.; Gooding, J. J. Challenges and Solutions 2665 in Developing Ultrasensitive Biosensors. J. Am. Chem. Soc. 2019, 141, $26661162-1170$.

2667 (145) Giamblanco, N.; Coglitore, D.; Janot, J.-M.; Coulon, P. E.; 2668 Charlot, B.; Balme, S. Detection of Protein Aggregate Morphology 2669 through Single Antifouling Nanopore. Sens. Actuators, B 2018, 260, $2670736-745$.

2671 (146) Pidhatika, B.; Rodenstein, M.; Chen, Y.; Rakhmatullina, E.; 2672 Mühlebach, A.; Acikgöz, C.; Textor, M.; Konradi, R. Comparative 2673 Stability Studies of Poly(2-methyl-2-oxazoline) and Poly(ethylene 2674 glycol) Brush Coatings. Biointerphases 2012, 7, 1.

2675 (147) Du, Y.; Gao, J. Y.; Chen, T. T.; Zhang, C.; Ji, J.; Xu, Z. K. 2676 Understanding the Oxidative Stability of Antifouling Polymer 2677 Brushes. Langmuir 2017, 33, 7298-7304.

2678 (148) Li, L.; Chen, S.; Jiang, S. Protein Interactions with 2679 Oligo(ethylene glycol) (OEG) Self-Assembled Monolayers: OEG
Stability, Surface Packing Density and Protein Adsorption. J. Biomater. 2680 Sci., Polym. Ed. 2007, 18, 1415-1427.

2681

(149) Ong, C. S.; Al-anzi, B.; Lau, W. J.; Goh, P. S.; Lai, G. S.; 2682 Ismail, A. F.; Ong, Y. S. Anti-Fouling Double-Skinned Forward 2683 Osmosis Membrane with Zwitterionic Brush for Oily Wastewater 2684 Treatment. Sci. Rep. 2017, 7, 6904.

2685

(150) Bai, T.; Sun, F.; Zhang, L.; Sinclair, A.; Liu, S. J.; Ella-Menye, 2686 J. R.; Zheng, Y.; Jiang, S. Y. Restraint of the Differentiation of 2687 Mesenchymal Stem Cells by a Nonfouling Zwitterionic Hydrogel. 2688 Angew. Chem., Int. Ed. 2014, 53, 12729-12734.

2689

(151) Estephan, Z. G.; Schlenoff, P. S.; Schlenoff, J. B. Zwitteration 2690 as an Alternative to PEGylation. Langmuir 2011, 27, 6794-6800. 2691 (152) Rodriguez Emmenegger, C.; Brynda, E.; Riedel, T.; Sedlakova, 2692 Z.; Houska, M.; Alles, A. B. Interaction of Blood Plasma with 2693 Antifouling Surfaces. Langmuir 2009, 25, 6328-6333.

2694

(153) Konai, M. M.; Bhattacharjee, B.; Ghosh, S.; Haldar, J. Recent 2695 Progress in Polymer Research to Tackle Infections and Antimicrobial 2696 Resistance. Biomacromolecules 2018, 19, 1888-1917. 2697

(154) Le, N. L.; Ulbricht, M.; Nunes, S. P. How Do Polyethylene 2698 Glycol and Poly(sulfobetaine) Hydrogel Layers on Ultrafiltration 2699 Membranes Minimize Fouling and Stay Stable in Cleaning 2700 Chemicals? Ind. Eng. Chem. Res. 2017, 56, 6785-6795.

2701

(155) Chang, Y.; Shih, Y. J.; Lai, C. J.; Kung, H. H.; Jiang, S. Y. 2702 Blood-Inert Surfaces via Ion-Pair Anchoring of Zwitterionic 2703 Copolymer Brushes in Human Whole Blood. Adv. Funct. Mater. 2704 2013, 23, 1100-1110.

2705

(156) Zhu, Y.; Xu, X.; Brault, N. D.; Keefe, A. J.; Han, X.; Deng, Y.; 2706 $\mathrm{Xu}$, J.; Yu, Q.; Jiang, S. Cellulose Paper Sensors Modified with 2707 Zwitterionic Poly(carboxybetaine) for Sensing and Detection in 2708 Complex Media. Anal. Chem. 2014, 86, 2871-2875. 2709

(157) Sam, S.; Touahir, L.; Salvador Andresa, J.; Allongue, P.; 2710 Chazalviel, J. N.; Gouget-Laemmel, A. C.; Henry de Villeneuve, C.; 2711 Moraillon, A.; Ozanam, F.; Gabouze, N.; et al. Semiquantitative Study 2712 of the EDC/NHS Activation of Acid Terminal Groups at Modified 2713 Porous Silicon Surfaces. Langmuir 2010, 26, 809-814. 2714

(158) Wang, Y.-S.; Yau, S.; Chau, L.-K.; Mohamed, A.; Huang, C.-J. 2715 Functional Biointerfaces Based on Mixed Zwitterionic Self-Assembled 2716 Monolayers for Biosensing Applications. Langmuir 2019, 35, 1652- 2717 1661. 2718

(159) Brault, N. D.; White, A. D.; Taylor, A. D.; Yu, Q.; Jiang, S. 2719 Directly Functionalizable Surface Platform for Protein Arrays in 2720 Undiluted Human Blood Plasma. Anal. Chem. 2013, 85, 1447-1453. 2721 (160) Qi, M.; Zhang, Y.; Cao, C.; Zhang, M.; Liu, S.; Liu, G. 2722 Decoration of Reduced Graphene Oxide Nanosheets with Aryldiazo- 2723 nium Salts and Gold Nanoparticles toward a Label-Free Ampero- 2724 metric Immunosensor for Detecting Cytokine Tumor Necrosis 2725 Factor- $\alpha$ in Live Cells. Anal. Chem. 2016, 88, 9614-9621. 2726 (161) Wei, H.; Ni, S.; Cao, C.; Yang, G.-F.; Liu, G. Graphene Oxides 2727 Signal Reporters Based Multifunctional Immunosensing Platform for 2728 Amperometric Profiling of Multiple Cytokines in Serum. ACS. Sens. 2729 2018, 3, 1553-1561.

2730

(162) Ederth, T.; Lerm, M.; Orihuela, B.; Rittschof, D. Resistance of 2731 Zwitterionic Peptide Monolayers to Biofouling. Langmuir 2019, 35, 2732 $1818-1827$.

2733

(163) Lin, P.; Ding, L.; Lin, C. W.; Gu, F. Nonfouling Property of 2734 Zwitterionic Cysteine Surface. Langmuir 2014, 30, 6497-6507. 2735 (164) Liu, N.; Hui, N.; Davis, J. J.; Luo, X. Low Fouling Protein 2736 Detection in Complex Biological Media Supported by a Designed 2737 Multifunctional Peptide. ACS. Sens. 2018, 3, 1210-1216. 2738

(165) Ye, H.; Wang, L.; Huang, R.; Su, R.; Liu, B.; Qi, W.; He, Z. 2739 Superior Antifouling Performance of a Zwitterionic Peptide 2740 Compared to an Amphiphilic, Non-Ionic Peptide. ACS Appl. Mater. 2741 Interfaces 2015, 7, 22448-22457.

2742

(166) Qian, H.; Huang, Y.; Duan, X.; Wei, X.; Fan, Y.; Gan, D.; Yue, 2743 S.; Cheng, W.; Chen, T. Fiber Optic Surface Plasmon Resonance 2744 Biosensor for Detection of PDGF-BB in Serum Based on Self- 2745 Assembled Aptamer and Antifouling Peptide Monolayer. Biosens. 2746 Bioelectron. 2019, 140, 111350. 
2748 (167) Wang, G.; Han, R.; Su, X.; Li, Y.; Xu, G.; Luo, X. Zwitterionic 2749 Peptide Anchored to Conducting Polymer PEDOT for the Develop2750 ment of Antifouling and Ultrasensitive Electrochemical DNA Sensor. 2751 Biosens. Bioelectron. 2017, 92, 396-401.

2752 (168) Liu, N.; Song, J.; Yanwei, L.; Davis, J. J.; Gao, F.; Luo, X. 2753 Electrochemical Aptasensor for Ultralow Fouling Cancer Cell 2754 Quantification in Complex Biological Media Based on Designed 2755 Branched Peptides. Anal. Chem. 2019, 91, 8334-8340.

2756 (169) Liu, X.; Huang, R.; Su, R.; Qi, W.; Wang, L.; He, Z. Grafting 2757 Hyaluronic Acid onto Gold Surface to Achieve Low Protein Fouling 2758 in Surface Plasmon Resonance Biosensors. ACS Appl. Mater. Interfaces 2759 2014, 6, 13034-13042.

2760 (170) Niu, Y. L.; Chu, M. L.; Xu, P.; Meng, S. S.; Zhou, Q.; Zhao, 2761 W. B.; Zhao, B.; Shen, J. An Aptasensor Based on Heparin-Mimicking 2762 Hyperbranched Polyester with Anti-biofouling Interface for Sensitive 2763 Thrombin Detection. Biosens. Bioelectron. 2018, 101, 174-180.

2764 (171) Moore, E.; Delalat, B.; Vasani, R.; McPhee, G.; Thissen, H.; 2765 Voelcker, N. H. Surface-Initiated Hyperbranched Polyglycerol as an 2766 Ultralow-Fouling Coating on Glass, Silicon, and Porous Silicon 2767 Substrates. ACS Appl. Mater. Interfaces 2014, 6, 15243-15252.

2768 (172) Moore, E.; Delalat, B.; Vasani, R.; Thissen, H.; Voelcker, N. 2769 H. Patterning and Biofunctionalization of Antifouling Hyperbranched 2770 Polyglycerol Coatings. Biomacromolecules 2014, 15, 2735-2743.

2771 (173) Chen, P. R.; Wang, T. C.; Chen, S. T.; Chen, H. Y.; Tsai, W. 2772 B. Development of Antifouling Hyperbranched Polyglycerol Layers 2773 on Hydroxyl Poly-p-xylylene Coatings. Langmuir 2017, 33, 14657277414662.

2775 (174) Liu, Z.; An, X.; Dong, C.; Zheng, S.; Mi, B.; Hu, Y. 2776 Modification of Thin Film Composite Polyamide Membranes with 2777 3D Hyperbranched Polyglycerol for Simultaneous Improvement in 2778 Their Filtration Performance and Antifouling Properties. J. Mater. 2779 Chem. A 2017, 5, 23190.

2780 (175) Ma, L.; Jayachandran, S.; Li, Z.; Song, Z.; Wang, W.; Luo, X. 2781 Antifouling and Conducting PEDOT Derivative Grafted with 2782 Polyglycerol for Highly Sensitive Electrochemical Protein Detection 2783 in Complex Biological Media. J. Electroanal. Chem. 2019, 840, 2722784278.

2785 (176) Qi, H.; Li, S.; Li, C.; Li, X.; Gao, Q.; Zhang, C. Sensitive and 2786 Antifouling Impedimetric Aptasensor for the Determination of 2787 Thrombin in Undiluted Serum Sample. Biosens. Bioelectron. 2013, 2788 39, 324-328.

2789 (177) McQuistan, A.; Zaitouna, A. J.; Echeverria, E.; Lai, R. Y. Use 2790 of Thiolated Oligonucleotides as Anti-Fouling Diluents in Electro2791 chemical Peptide-Based Sensors. Chem. Commun. 2014, 50, 469027924692.

2793 (178) Hui, 1.; Xu, A.; Liu, H. DNA-Based Nanofabrication for 2794 Antifouling Applications. Langmuir 2019, 35, 12543-12549.

2795 (179) Subbiahdoss, G.; Zeng, G.; Aslan, H.; Ege Friis, J.; Iruthayaraj, 2796 J.; Zelikin, A. N.; Meyer, R. L. Antifouling Properties of Layer by 2797 Layer DNA Coatings. Biofouling 2019, 35, 75-88.

2798 (180) Nie, W.; Wang, Q.; Zou, L.; Zheng, Y.; Liu, X.; Yang, X.; 2799 Wang, K. Low-Fouling Surface Plasmon Resonance Sensor for Highly 2800 Sensitive Detection of MicroRNA in a Complex Matrix Based on the 2801 DNA Tetrahedron. Anal. Chem. 2018, 90, 12584-12591.

2802 (181) McKeating, K. S.; Hinman, S. S.; Rais, N. A.; Zhou, Z.; Cheng, 2803 Q. Antifouling Lipid Membranes over Protein A for Orientation2804 Controlled Immunosensing in Undiluted Serum and Plasma. ACS. 2805 Sens. 2019, 4, 1774-1782.

2806 (182) Hu, C.-M. J.; Fang, R. H.; Wang, K.-C.; Luk, B. T.; 2807 Thamphiwatana, S.; Dehaini, D.; Nguyen, P.; Angsantikul, P.; Wen, C. 2808 H.; Kroll, A. V.; et al. Nanoparticle Biointerfacing by Platelet 2809 Membrane Cloaking. Nature 2015, 526, 118-121.

2810 (183) Piao, J.-G.; Wang, L.; Gao, F.; You, Y.-Z.; Xiong, Y.; Yang, L. 2811 Erythrocyte Membrane Is an Alternative Coating to Polyethylene 2812 Glycol for Prolonging the Circulation Lifetime of Gold Nanocages for 2813 Photothermal Therapy. ACS Nano 2014, 8, 10414-10425.

2814 (184) Fan, B.; Fan, Q.; Cui, M.; Wu, T.; Wang, J.; Ma, H.; Wei, Q. 2815 Photoelectrochemical Biosensor for Sensitive Detection of Soluble 2816 CD44 Based on the Facile Construction of a Poly(ethylene glycol)/
Hyaluronic Acid Hybrid Antifouling Interface. ACS Appl. Mater. 2817 Interfaces 2019, 11, 24764-24770.

(185) Xia, Y.; Adibnia, V.; Shan, C.; Huang, R.; Qi, W.; He, Z.; Xie, 2819 G.; Olszewski, M.; De Crescenzo, G.; Matyjaszewski, K.; et al. 2820 Synergy between Zwitterionic Polymers and Hyaluronic Acid 2821 Enhances Antifouling Performance. Langmuir 2019, 35, 15535- 2822 15542 .

2823

(186) Chen, L.; Zhao, S.; Liu, M.; Wu, P.; Chen, C. A Novel Label- 2824 Free Electrochemical Immunosensor Modified by Glutathione and 2825 Hyaluronic Acid for the Ultrasensitive and Ultraselective Detection of 2826 Brucellosis in Dilute Serum Done. Sens. Actuators, B 2019, 287, 510- 2827 516.

2828

(187) Kim, S.; Lee, S.; Park, J.; Lee, J. Y. Electrochemical Co- 2829 deposition of Polydopamine/Hyaluronic Acid for Anti-Biofouling 2830 Bioelectrodes. Front. Chem. 2019, 7, 262.

2831

(188) Ye, H.; Xia, Y.; Liu, Z.; Huang, R.; Su, R.; Qi, W.; Wang, L.; 2832 $\mathrm{He}, \mathrm{Z}$. Dopamine-Assisted Deposition and Zwitteration of Hyaluronic 2833 Acid for the Nanoscale Fabrication of Low-Fouling Surfaces. J. Mater. 2834 Chem. B 2016, 4, 4084-4091.

2835

(189) Ye, H.; Che, J.; Huang, R.; Qi, W.; He, Z.; Su, R. Zwitterionic 2836 Peptide Enhances Protein-Resistant Performance of Hyaluronic Acid- 2837 Modified Surfaces. Langmuir 2020, 36, 1923-1929.

2838

(190) Wei, Q.; Liu, X.; Yue, Q.; Ma, S.; Zhou, F. Mussel-Inspired 2839 One-Step Fabrication of Ultralow-Friction Coatings on Diverse 2840 Biomaterial Surfaces. Langmuir 2019, 35, 8068-8075. 2841

(191) Xia, Y.; Adibnia, V.; Huang, R.; Murschel, F.; Faivre, J.; Xie, 2842 G.; Olszewski, M.; De Crescenzo, G.; Qi, W.; He, Z.; et al. 2843 Biomimetic Bottlebrush Polymer Coatings for Fabrication of Ultralow 2844 Fouling Surfaces. Angew. Chem. 2019, 131, 1322-1328. 2845

(192) Ye, H.; Han, M.; Huang, R.; Schmidt, T. A.; Qi, W.; He, Z.; 2846 Martin, L. L.; Jay, G. D.; Su, R.; Greene, G. W. Interactions between 2847 Lubricin and Hyaluronic Acid Synergistically Enhance Antiadhesive 2848 Properties. ACS Appl. Mater. Interfaces 2019, 11, 18090-18102. 2849 (193) Liu, G.; Qi, M.; Zhang, Y.; Cao, C.; Goldys, E. M. 2850 Nanocomposites of Gold Nanoparticles and Graphene Oxide towards 2851 an Stable Label-free Electrochemical Immunosensor for Detection of 2852 Cardiac Marker Troponin-I. Anal. Chim. Acta 2016, 909, 1-8. 2853

(194) Wang, G. Z.; Wang, L. G.; Lin, W. F.; Wang, Z.; Zhang, J.; Ji, 2854 F. Q.; Ma, G. L.; Yuan, Z. F.; Chen, S. F. Development of Robust and 2855 Recoverable Ultralow-Fouling Coatings Based on Poly- 2856 (carboxybetaine) Ester Analogue. ACS Appl. Mater. Interfaces 2015, 2857 7, 16938-16945.

2858

(195) Sharma, R.; Deacon, S. E.; Nowak, D.; George, S.; Szymonik, 2859 M.; Tang, A.; Tomlinson, D.; Davies, A.; McPherson, M.; Wälti, C. 2860 Label-Free Electrochemical Impedance Biosensor to Detect Human 2861 Interleukin-8 in Serum with Sub-pg/mL Sensitivity. Biosens. 2862 Bioelectron. 2016, 80, 607-613.

2863

(196) Lv, S.; Sheng, J.; Zhao, S.; Liu, M.; Chen, L. The Detection of 2864 Brucellosis Antibody in Whole Serum Based on the Low-fouling 2865 Electrochemical Immunosensor Fabricated with Magnetic $\mathrm{Fe}_{3} \mathrm{O}_{4} @ 2866$ Au@ PEG@HA Nanoparticles. Biosens. Bioelectron. 2018, 117, 138- 2867 144.

$$
2868
$$

(197) Bayramoglu, G.; Ozalp, C.; Oztekin, M.; Guler, U.; Salih, B.; 2869 Arica, M. Y. Design of an Aptamer-Based Magnetic Adsorbent and 2870 Biosensor Systems for Selective and Sensitive Separation and 2871 Detection of Thrombin. Talanta 2019, 191, 59-66.

2872

(198) Mathew, D.; Beekman, P.; Lemay, S. G.; Zuilhof, H.; Le Gac, 2873 S.; van der Wiel, W. G. Electrochemical Detection of Tumor-Derived 2874 Extracellular Vesicles on Nano-interdigitated Electrodes. Nano Lett. 2875 2020, 20, 820-828.

2876

(199) He, X.; Han, H.; Liu, L.; Shi, W.; Lu, X.; Dong, J.; Yang, W.; 2877 $\mathrm{Lu}, \mathrm{X}$. Self-Assembled Microgels for Sensitive and Low-Fouling 2878 Detection of Streptomycin in Complex Media. ACS Appl. Mater. 2879 Interfaces 2019, 11, 13676-13684.

2880

(200) Cui, M.; Wang, Y.; Jiao, M.; Jayachandran, S.; Wu, Y.; Fan, X.; 2881 Luo, X. Mixed Self-Assembled Aptamer and Newly Designed 2882 Zwitterionic Peptide as Antifouling Biosensing Interface for Electro- 2883 chemical Detection of alpha-Fetoprotein. ACS. Sens. 2017, 2, 490- 2884 494. 
2886 (201) Li, Y.; Wang, L.; Ding, C.; Luo, X. Highly Selective 2887 Ratiometric Electrogenerated Chemiluminescence Assay of DNA 2888 Methyltransferase Activity via Polyaniline and Anti-Fouling Peptide 2889 Modified Electrode. Biosens. Bioelectron. 2019, 142, 111553.

2890 (202) Wang, Y.; Cui, M.; Jiao, M.; Luo, X. Antifouling and 2891 Ultrasensitive Biosensing Interface Based on Self-Assembled Peptide 2892 and Aptamer on Macroporous Gold for Electrochemical Detection of 2893 Immunoglobulin E in Serum. Anal. Bioanal. Chem. 2018, 410, 587128945878.

2895 (203) Fan, G.-C.; Li, Z.; Lu, Y.; Ma, L.; Zhao, H.; Luo, X. Robust 2896 Photoelectrochemical Cytosensor in Biological Media Using Anti2897 fouling Property of Zwitterionic Peptide. Sens. Actuators, B 2019, 299, 2898126996.

2899 (204) Song, Z.; Li, Y.; Teng, H.; Ding, C.; Xu, G.; Luo, X. Designed 2900 Zwitterionic Peptide Combined with Sacrificial Fe-MOF for Low 2901 Fouling and Highly Sensitive Electrochemical Detection of T4 2902 Polynucleotide Kinase. Sens. Actuators, B 2020, 305, 127329.

2903 (205) Chang, P.-H.; Weng, C.-C.; Li, B.-R.; Li, Y.-K. An Antifouling 2904 Peptide-Based Biosensor for Determination of Streptococcus 2905 Pneumonia Markers in Human Serum. Biosens. Bioelectron. 2020, $2906151,111969$.

2907 (206) Xu, Q.; Wang, G.; Zhang, M.; Xu, G.; Lin, J.; Luo, X. Aptamer 2908 Based Label Free Thrombin Assay Based on the Use of Silver 2909 Nanoparticles Incorporated into Self-Polymerized Dopamine. Micro2910 chim. Acta 2018, 185, 253.

2911 (207) Wang, W.; Cui, M.; Song, Z.; Luo, X. An Antifouling 2912 Electrochemical Immunosensor for Carcinoembryonic Antigen Based 2913 on Hyaluronic Acid Doped Conducting Polymer PEDOT. RSC Adv. 2914 2016, 6, 88411-88416.

2915 (208) Liu, Z.; Wang, H. An Antifouling Interface Integrated with 2916 HRP-Based Amplification to Achieve Highly Sensitive Electro2917 chemical Aptasensor for Lysozyme Detection. Analyst 2019, 144, 2918 5794-5801.

2919 (209) Baradoke, A.; Hein, R.; Li, X.; Davis, J. J. Reagentless Redox 2920 Capacitive Assaying of C-Reactive Protein at a Polyaniline Interface. 2921 Anal. Chem. 2020, 92, 3508-3511.

2922 (210) Soto, R. J.; Hall, J. R.; Brown, M. D.; Taylor, J. B.; 2923 Schoenfisch, M. H. In Vivo Chemical Sensors: Role of Biocompat2924 ibility on Performance and Utility. Anal. Chem. 2017, 89, 276-299. 2925 (211) Rong, G.; Corrie, S. R.; Clark, H. A. In Vivo Biosensing: 2926 Progress and Perspectives. ACS. Sens. 2017, 2, 327-338.

2927 (212) Liu, J.; Yu, M.; Ning, X.; Zhou, C.; Yang, S.; Zheng, J. 2928 PEGylation and Zwitterionization: Pros and Cons in the Renal 2929 Clearance and Tumor Targeting of Near-IR-Emitting Gold Nano2930 particles. Angew. Chem. 2013, 125, 12804-12808.

2931 (213) Ruckh, T. T.; Clark, H. A. Implantable Nanosensors: Toward 2932 Continuous Physiologic Monitoring. Anal. Chem. 2014, 86, 131429331323.

2934 (214) Chatard, C.; Meiller, A.; Marinesco, S. Microelectrode 2935 Biosensors for in Vivo Analysis of Brain Interstitial Fluid. Electro2936 analysis 2018, 30, 977-998.

2937 (215) Dardano, P.; Rea, I.; De Stefano, L. Microneedles Based 2938 Electrochemical Sensors: New Tools for Advanced Biosensing. Curr. 2939 Opin. Electrochem. 2019, 17, 121-127.

2940 (216) Baranwal, A.; Chandra, P. Clinical Implications and 2941 Electrochemical Biosensing of Monoamine Neurotransmitters in 2942 Body Fluids, in Vitro, in Vivo, and Ex Vivo Models. Biosens. 2943 Bioelectron. 2018, 121, 137-152.

2944 (217) Xiao, T.; Wu, F.; Hao, J.; Zhang, M.; Yu, P.; Mao, L. In Vivo 2945 Analysis with Electrochemical Sensors and Biosensors. Anal. Chem. 2946 2017, 89, 300-313.

2947 (218) Shibata, H.; Heo, Y. J.; Okitsu, T.; Matsunaga, Y.; Kawanishi, 2948 T.; Takeuchi, S. Injectable Hydrogel Microbeads for Fluorescence2949 Based in vivo Continuous Glucose Monitoring. Proc. Natl. Acad. Sci. 2950 U. S. A. 2010, 107, 17894-17898.

2951 (219) He, Q.; Zhang, Z.; Gao, F.; Li, Y.; Shi, J. In vivo 2952 Biodistribution and Urinary Excretion of Mesoporous Silica Nano2953 particles: Effects of Particle Size and PEGylation. Small 2011, 7, 2712954280.
(220) Dudani, J. S.; Jain, P. K.; Kwong, G. A.; Stevens, K. R.; Bhatia, 2955 S. N. Photoactivated Spatiotemporally-Responsive Nanosensors of in 2956 Vivo Protease Activity. ACS Nano 2015, 9, 11708-11717. 2957 (221) Wei, R.; Cai, Z.; Ren, B. W.; Li, A.; Lin, H.; Zhang, K.; Chen, 2958 H.; Shan, H.; Ai, H.; Gao, J. Biodegradable and Renal-Clearable 2959 Hollow Porous Iron Oxide Nanoboxes for In Vivo Imaging. Chem. 2960 Mater. 2018, 30, 7950-7961.

2961

(222) Liu, W.; Choi, H. S.; Zimmer, J. P.; Tanaka, E.; Frangioni, J. 2962 V.; Bawendi, M. Compact Cysteine-Coated CdSe(ZnCdS) Quantum 2963 Dots for in Vivo Applications. J. Am. Chem. Soc. 2007, 129, 14530- 2964 14531.

2965

(223) Soo Choi, H.; Liu, W.; Misra, P.; Tanaka, E.; Zimmer, J. P.; 2966 Itty Ipe, B.; Bawendi, M. G.; Frangioni, J. V. Renal Clearance of 2967 Quantum Dots. Nat. Biotechnol. 2007, 25, 1165.

2968

(224) Breus, V. V.; Heyes, C. D.; Tron, K.; Nienhaus, G. U. 2969 Zwitterionic Biocompatible Quantum Dots for Wide pH Stability and 2970 Weak Nonspecific Binding to Cells. ACS Nano 2009, 3, 2573-2580. 2971 (225) Kozai, T. D. Y.; Langhals, N. B.; Patel, P. R.; Deng, X.; Zhang, 2972 H.; Smith, K. L.; Lahann, J.; Kotov, N. A.; Kipke, D. R. Ultrasmall 2973 Implantable Composite Microelectrodes with Bioactive Surfaces for 2974 Chronic Neural Interfaces. Nat. Mater. 2012, 11, 1065-1073. 2975

(226) Schwerdt, H. N.; Zhang, E.; Kim, M. J.; Yoshida, T.; 2976 Stanwicks, L.; Amemori, S.; Dagdeviren, H. E.; Langer, R.; Cima, M. 2977 J.; Graybiel, A. M. Cellular-Scale Probes Enable Stable Chronic 2978 Subsecond Monitoring of Dopamine Neurochemicals in a Rodent 2979 Model. Commun. Biol. 2018, 1, 144.

(227) Obidin, N.; Tasnim, F.; Dagdeviren, C. The Future of 2981 Neuroimplantable Devices: A Materials Science and Regulatory 2982 Perspective. Adv. Mater. 2019, 0, 201901482.

2983

(228) Huang, Y.; Geng, X.; Li, L.; Stein, J. F.; Aziz, T. Z.; Green, A. 2984 L.; Wang, S. Measuring Complex Behaviors of Local Oscillatory 2985 Networks in Deep Brain Local Field Potentials. J. Neurosci. Methods 2986 2016, 264, 25-32.

(229) Huang, Y.; Luo, H.; Green, A. L.; Aziz, T. Z.; Wang, S. 2988 Characteristics of Local Field Potentials Correlate with Pain Relief by 2989 Deep Brain Stimulation. Clin. Neurophysiol. 2016, 127, 2573-2580. 2990 (230) Huang, Y.; Green, A. L.; Hyam, J.; Fitzgerald, J.; Aziz, T. Z.; 2991 Wang, S. Oscillatory Neural Representations in the Sensory Thalamus 2992 Predict Neuropathic Pain Relief by Deep Brain Stimulation. Neurobiol. 2993 Dis. 2018, 109, 117-126.

2994

(231) Machado, R.; Soltani, N.; Dufour, S.; Salam, M.; Carlen, P.; 2995 Genov, R.; Thompson, M. Biofouling-Resistant Impedimetric Sensor 2996 for Array High-Resolution Extracellular Potassium Monitoring in the 2997 Brain. Biosensors 2016, 6, 53.

2998

(232) Smart, S. L.; Lopantsev, V.; Zhang, C.; Robbins, C. A.; Wang, 2999 H.; Chiu, S.; Schwartzkroin, P. A.; Messing, A.; Tempel, B. L. 3000 Deletion of the Kv1. 1 Potassium Channel Causes Epilepsy in Mice. 3001 Neuron 1998, 20, 809-819.

3002

(233) Robinson, K. J.; Huynh, G. T.; Kouskousis, B. P.; Fletcher, N. 3003 L.; Houston, Z. H.; Thurecht, K. J.; Corrie, S. R. Modified 3004 Organosilica Core-Shell Nanoparticles for Stable $\mathrm{pH}$ Sensing in 3005 Biological Solutions. ACS. Sens. 2018, 3, 967-975. 3006

(234) Miller, C. R.; Vogel, R.; Surawski, P. P. T.; Jack, K. S.; Corrie, 3007 S. R.; Trau, M. Functionalized Organosilica Microspheres via a Novel 3008 Emulsion-Based Route. Langmuir 2005, 21, 9733-9740. 3009

(235) Cash, K. J.; Clark, H. A. Phosphorescent Nanosensors for In 3010 Vivo Tracking of Histamine Levels. Anal. Chem. 2013, 85, 6312- 3011 6318.

(236) Dubach, J. M.; Harjes, D. I.; Clark, H. A. Fluorescent Ion- 3013 Selective Nanosensors for Intracellular Analysis with Improved 3014 Lifetime and Size. Nano Lett. 2007, 7, 1827-1831. 3015

(237) Querfurth, H. W.; LaFerla, F. M. Alzheimer's Disease. N. Engl. 3016 J. Med. 2010, 362, 329-344.

3017

(238) Zhen, X.; Zhang, C.; Xie, C.; Miao, Q.; Lim, K. L.; Pu, K. 3018 Intraparticle Energy Level Alignment of Semiconducting Polymer 3019 Nanoparticles to Amplify Chemiluminescence for Ultrasensitive In 3020 Vivo Imaging of Reactive Oxygen Species. ACS Nano 2016, 10, 3021 6400-6409. 
3023 (239) Shuhendler, A. J.; Pu, K.; Cui, L.; Uetrecht, J. P.; Rao, J. Real3024 Time Imaging of Oxidative and Nitrosative Stress in the Liver of Live 3025 Animals for Drug-Toxicity Testing. Nat. Biotechnol. 2014, 32, 3733026380 .

3027 (240) Xie, J.; Zhang, F.; Aronova, M.; Zhu, L.; Lin, X.; Quan, Q.; 3028 Liu, G.; Zhang, G.; Choi, K.-Y.; Kim, K.; et al. Manipulating the 3029 Power of an Additional Phase: A Flower-like $\mathrm{Au}-\mathrm{Fe}_{3} \mathrm{O}_{4}$ Optical 3030 Nanosensor for Imaging Protease Expressions In Vivo. ACS Nano 3031 2011, 5, 3043-3051.

3032 (241) Abu Lila, A. S.; Kiwada, H.; Ishida, T. The Accelerated Blood 3033 Clearance $(\mathrm{ABC})$ Phenomenon: Clinical Challenge and Approaches 3034 to Manage. J. Controlled Release 2013, 172, 38-47.

3035 (242) Schellekens, H.; Hennink, W. E.; Brinks, V. The 3036 Immunogenicity of Polyethylene Glycol: Facts and Fiction. Pharm. 3037 Res. 2013, 30, 1729-1734.

3038 (243) Herzenberg, L. A.; Tokuhisa, T. Epitope-specific regulation. I. 3039 Carrier-Specific Induction of Suppression for IgG Anti-Hapten 3040 Antibody Responses. J. Exp. Med. 1982, 155, 1730-1740.

3041 (244) Liu, X.; Xiao, T.; Wu, F.; Shen, M. Y.; Zhang, M.; Yu, H. h.; 3042 Mao, L. Ultrathin Cell-Membrane-Mimic Phosphorylcholine Polymer 3043 Film Coating Enables Large Improvements for In Vivo Electro3044 chemical Detection. Angew. Chem., Int. Ed. 2017, 56, 11802-11806. 3045 (245) Qi, M.; Huang, J.; Wei, H.; Cao, C.; Feng, S.; Guo, Q.; 3046 Goldys, E. M.; Li, R.; Liu, G. Graphene Oxide Thin Film with Dual 3047 Function Integrated into a Nanosandwich Device for In Vivo 3048 Monitoring of Interleukin-6. ACS Appl. Mater. Interfaces 2017, 9, 3049 41659-41668.

3050 (246) Li, H.; Dauphin-Ducharme, P.; Arroyo-Currás, N.; Tran, C. 3051 H.; Vieira, P. A.; Li, S.; Shin, C.; Somerson, J.; Kippin, T. E.; Plaxco, 3052 K. W. A Biomimetic Phosphatidylcholine-Terminated Monolayer 3053 Greatly Improves the In Vivo Performance of Electrochemical 3054 Aptamer-Based Sensors. Angew. Chem., Int. Ed. 2017, 56, 7492-7495. 3055 (247) Baker, B. R.; Lai, R. Y.; Wood, M. S.; Doctor, E. H.; Heeger, 3056 A. J.; Plaxco, K. W. An Electronic, Aptamer-Based Small-Molecule 3057 Sensor for the Rapid, Label-Free Detection of Cocaine in Adulterated 3058 Samples and Biological Fluids. J. Am. Chem. Soc. 2006, 128, 313830593139.

3060 (248) Zuo, X.; Xiao, Y.; Plaxco, K. W. High Specificity, 3061 Electrochemical Sandwich Assays Based on Single Aptamer 3062 Sequences and Suitable for the Direct Detection of Small-Molecule 3063 Targets in Blood and Other Complex Matrices. J. Am. Chem. Soc. 3064 2009, 131, 6944-6945.

3065 (249) Hamburg, M. A.; Collins, F. S. The Path to Personalized 3066 Medicine. N. Engl. J. Med. 2010, 363, 301-304.

3067 (250) Li, X.; Deng, D.; Xue, J.; Qu, L.; Achilefu, S.; Gu, Y. Quantum 3068 Dots Based Molecular Beacons for In Vitro and In Vivo Detection of 3069 MMP-2 on Tumor. Biosens. Bioelectron. 2014, 61, 512-518.

3070 (251) Choi, Y.; Kim, S.; Choi, M.-H.; Ryoo, S.-R.; Park, J.; Min, D.3071 H.; Kim, B.-S. Highly Biocompatible Carbon Nanodots for 3072 Simultaneous Bioimaging and Targeted Photodynamic Therapy In 3073 Vitro and In Vivo. Adv. Funct. Mater. 2014, 24, 5781-5789.

3074 (252) Pu, K.; Shuhendler, A. J.; Rao, J. Semiconducting Polymer 3075 Nanoprobe for In Vivo Imaging of Reactive Oxygen and Nitrogen 3076 Species. Angew. Chem., Int. Ed. 2013, 52, 10325-10329.

3077 (253) Iverson, N. M.; Barone, P. W.; Shandell, M.; Trudel, L. J.; Sen, 3078 S.; Sen, F.; Ivanov, V.; Atolia, E.; Farias, E.; McNicholas, T. P.; et al. 3079 In Vivo Biosensing via Tissue-Localizable Near-Infrared-Fluorescent 3080 Single-Walled Carbon Nanotubes. Nat. Nanotechnol. 2013, 8, 873. 3081 (254) Xiong, L.; Shuhendler, A. J.; Rao, J. Self-Luminescing BRET3082 FRET Near-Infrared Dots for In Vivo Lymph-Node Mapping and 3083 Tumour Imaging. Nat. Commun. 2012, 3, 1193.

3084 (255) He, R.; Niu, Y.; Li, Z.; Li, A.; Yang, H.; Xu, F.; Li, F. A 3085 Hydrogel Microneedle Patch for Point-of-Care Testing Based on Skin 3086 Interstitial Fluid. Adv. Healthcare Mater. 2020, 9, 1901201.

3087 (256) Zhou, L.; Hou, H.; Wei, H.; Yao, L.; Sun, L.; Yu, P.; Su, B.; 3088 Mao, L. In Vivo Monitoring of Oxygen in Rat Brain by Carbon Fiber 3089 Microelectrode Modified with Antifouling Nanoporous Membrane. 3090 Anal. Chem. 2019, 91, 3645-3651.
(257) Hao, J.; Xiao, T.; Wu, F.; Yu, P.; Mao, L. High Antifouling 3091 Property of Ion-Selective Membrane: Toward In Vivo Monitoring of 3092 pH Change in Live Brain of Rats with Membrane-Coated Carbon 3093 Fiber Electrodes. Anal. Chem. 2016, 88, 11238-11243. 3094

(258) Feng, T.; Ji, W.; Tang, Q.; Wei, H.; Zhang, S.; Mao, J.; Zhang, 3095 Y.; Mao, L.; Zhang, M. Low-Fouling Nanoporous Conductive 3096 Polymer-Coated Microelectrode for In Vivo Monitoring of Dopamine 3097 in the Rat Brain. Anal. Chem. 2019, 91, 10786-10791. 3098 (259) Ferguson, B. S.; Hoggarth, D. A.; Maliniak, D.; Ploense, K.; 3099 White, R. J.; Woodward, N.; Hsieh, K.; Bonham, A. J.; Eisenstein, M.; 3100 Kippin, T. E.; et al. Real-Time, Aptamer-Based Tracking of 3101 Circulating Therapeutic Agents in Living Animals. Sci. Transl. Med. 3102 2013, 5, 213ra165.

(260) Arroyo-Currás, N.; Somerson, J.; Vieira, P. A.; Ploense, K. L.; 3104 Kippin, T. E.; Plaxco, K. W. Real-Time Measurement of Small 3105 Molecules Directly in Awake, Ambulatory Animals. Proc. Natl. Acad. 3106 Sci. U. S. A. 2017, 114, 645-650.

3107

(261) Rivas, L.; Dulay, S.; Miserere, S.; Pla, L.; Marin, S. B.; Parra, J.; 3108 Eixarch, E.; Gratacós, E.; Illa, M.; Mir, M.; et al. Micro-Needle 3109 Implantable Electrochemical Oxygen Sensor: Ex-Vivo and In-Vivo 3110 Studies. Biosens. Bioelectron. 2020, 153, 112028.

(262) Vreeland, R. F.; Atcherley, C. W.; Russell, W. S.; Xie, J. Y.; Lu, 3112 D.; Laude, N. D.; Porreca, F.; Heien, M. L. Biocompatible 3113 PEDOT:Nafion Composite Electrode Coatings for Selective 3114 Detection of Neurotransmitters In Vivo. Anal. Chem. 2015, 87, 3115 2600-2607.

3116

(263) Patel, J.; Radhakrishnan, L.; Zhao, B.; Uppalapati, B.; Daniels, 3117 R. C.; Ward, K. R.; Collinson, M. M. Electrochemical Properties of 3118 Nanostructured Porous Gold Electrodes in Biofouling Solutions. Anal. 3119 Chem. 2013, 85, 11610-11618.

3120

(264) Daggumati, P.; Matharu, Z.; Seker, E. Effect of Nanoporous 3121 Gold Thin Film Morphology on Electrochemical DNA Sensing. Anal. 3122 Chem. 2015, 87, 8149-8156.

3123

(265) Chapman, C. A. R.; Chen, H.; Stamou, M.; Biener, J.; Biener, 3124 M. M.; Lein, P. J.; Seker, E. Nanoporous Gold as a Neural Interface 3125 Coating: Effects of Topography, Surface Chemistry, and Feature Size. 3126 ACS Appl. Mater. Interfaces 2015, 7, 7093-7100. 3127

(266) Daggumati, P.; Matharu, Z.; Wang, L.; Seker, E. Biofouling- 3128 Resilient Nanoporous Gold Electrodes for DNA Sensing. Anal. Chem. 3129 2015, 87, 8618-8622.

3130

(267) Liu, Z.; Zhang, H.; Hou, S.; Ma, H. Highly Sensitive and 3131 Selective Electrochemical Detection of L-Cysteine Using Nanoporous 3132 Gold. Microchim. Acta 2012, 177, 427-433.

3133

(268) Silva, T. A.; Khan, M. R. K.; Fatibello-Filho, O.; Collinson, M. 3134 M. Simultaneous Electrochemical Sensing of Ascorbic Acid and Uric 3135 Acid under Biofouling Conditions Using Nanoporous Gold Electro- 3136 des. J. Electroanal. Chem. 2019, 846, 113160. (269) Wu, S.; Lan, X.; Huang, F.; Luo, Z.; Ju, H.; Meng, C.; Duan, 3138 C. Selective Electrochemical Detection of Cysteine in Complex Serum 3139 by Graphene Nanoribbon. Biosens. Bioelectron. 2012, 32, 293-296. 3140 (270) Sun, Q.; Yan, F.; Yao, L.; Su, B. Anti-Biofouling Isoporous 3141 Silica-Micelle Membrane Enabling Drug Detection in Human Whole 3142 Blood. Anal. Chem. 2016, 88, 8364-8368.

3143

(271) Zhou, J.; Zhang, L.; Tian, Y. Micro Electrochemical pH 3144 Sensor Applicable for Real-Time Ratiometric Monitoring of $\mathrm{pH} 3145$ Values in Rat Brains. Anal. Chem. 2016, 88, 2113-2118. 3146

(272) Zhou, L.; Ding, H.; Yan, F.; Guo, W.; Su, B. Electrochemical 3147 Detection of Alzheimer's Disease Related Substances in Biofluids by 3148 Silica Nanochannel Membrane Modified Glassy Carbon Electrodes. 3149 Analyst 2018, 143, 4756-4763.

3150

(273) Yan, F.; Zheng, W.; Yao, L.; Su, B. Direct Electrochemical 3151 Analysis in Complex Samples Using ITO Electrodes Modified with 3152 Permselective Membranes Consisting of Vertically Ordered Silica 3153 Mesochannels and Micelles. Chem. Commun. 2015, 51, 17736- 3154 17739 .

3155

(274) Zhang, L.; Xu, J.; Tang, Y.; Hou, J.; Yu, L.; Gao, C. A Novel 3156 Long-Lasting Antifouling Membrane Modified with Bifunctional 3157 Capsaicin-Mimic Moieties via in Situ Polymerization for Efficient 3158 water Purification. J. Mater. Chem. A 2016, 4, 10352-10362. 
3160 (275) Kongsuphol, P.; Ng, H. H.; Pursey, J. P.; Arya, S. K.; Wong, C. 3161 C.; Stulz, E.; Park, M. K. EIS-Based Biosensor for Ultra-Sensitive 3162 Detection of TNF- $\alpha$ from Non-Diluted Human Serum. Biosens. 3163 Bioelectron. 2014, 61, 274-279.

3164 (276) Mei, N.; Seale, B.; Ng, A. H. C.; Wheeler, A. R.; Oleschuk, R. 3165 Digital Microfluidic Platform for Human Plasma Protein Depletion. 3166 Anal. Chem. 2014, 86, 8466-8472.

3167 (277) Olsen, S. M.; Pedersen, L. T.; Laursen, M.; Kiil, S.; Dam3168 Johansen, K. Enzyme-Based Antifouling Coatings: A Review. 3169 Biofouling 2007, 23, 369-383.

3170 (278) Kristensen, J. B.; Meyer, R. L.; Laursen, B. S.; Shipovskov, S.; 3171 Besenbacher, F.; Poulsen, C. H. Antifouling Enzymes and the 3172 Biochemistry of Marine Settlement. Biotechnol. Adv. 2008, 26, 4713173481.

3174 (279) Shi, Q.; Su, Y.; Ning, X.; Chen, W.; Peng, J.; Jiang, Z. Trypsin3175 Enabled Construction of Anti-Fouling and Self-Cleaning Polyether3176 sulfone Membrane. Bioresour. Technol. 2011, 102, 647-651.

3177 (280) Koseoglu-Imer, D. Y.; Dizge, N.; Koyuncu, I. Enzymatic 3178 Activation of Cellulose Acetate Membrane for Reducing of Protein 3179 Fouling. Colloids Surf., B 2012, 92, 334-339.

3180 (281) Harreither, W.; Trouillon, R.; Poulin, P.; Neri, W.; Ewing, A. 3181 G.; Safina, G. Cysteine Residues Reduce the Severity of Dopamine 3182 Electrochemical Fouling. Electrochim. Acta 2016, 210, 622-629.

3183 (282) Zhang, L.; Cao, Z.; Bai, T.; Carr, L.; Ella-Menye, J.-R.; Irvin, 3184 C.; Ratner, B. D.; Jiang, S. Zwitterionic Hydrogels Implanted in Mice 3185 Resist the Foreign-Body Reaction. Nat. Biotechnol. 2013, 31, 5533186556.

3187 (283) Zhong, S.; Campoccia, D.; Doherty, P.; Williams, R.; 3188 Benedetti, L.; Williams, D. Biodegradation of Hyaluronic Acid 3189 Derivatives by Hyaluronidase. Biomaterials 1994, 15, 359-365.

3190 (284) Zhang, L.; Chang, H.; Hirata, A.; Wu, H.; Xue, Q.-K.; Chen, 3191 M. Nanoporous Gold Based Optical Sensor for Sub-ppt Detection of 3192 Mercury Ions. ACS Nano 2013, 7, 4595-4600.

3193 (285) Chapman, R. G.; Ostuni, E.; Yan, L.; Whitesides, G. M. 3194 Preparation of Mixed Self-Assembled Monolayers (SAMs) That 3195 Resist Adsorption of Proteins Using the Reaction of Amines with a 3196 SAM That Presents Interchain Carboxylic Anhydride Groups. 3197 Langmuir 2000, 16, 6927-6936.

3198 (286) Liu; Gooding, J. J. An Interface Comprising Molecular Wires 3199 and Poly(ethylene glycol) Spacer Units Self-Assembled on Carbon 3200 Electrodes for Studies of Protein Electrochemistry. Langmuir 2006, $320122,7421-7430$.

3202 (287) Li, B.; Jain, P.; Ma, J.; Smith, J. K.; Yuan, Z.; Hung, H.-C.; He, 3203 Y.; Lin, X.; Wu, K.; Pfaendtner, J.; et al. Trimethylamine N-Oxide3204 Derived Zwitterionic Polymers: A New Class of Ultralow Fouling 3205 Bioinspired Materials. Sci. Adv. 2019, 5, No. eaaw9562.

3206 (288) Chelmowski, R.; Köster, S. D.; Kerstan, A.; Prekelt, A.; 3207 Grunwald, C.; Winkler, T.; Metzler-Nolte, N.; Terfort, A.; Wöll, C. 3208 Peptide-Based SAMs that Resist the Adsorption of Proteins. J. Am. 3209 Chem. Soc. 2008, 130, 14952-14953.

3210 (289) Sabaté del Río, J.; Henry, O. Y. F.; Jolly, P.; Ingber, D. E. An 3211 Antifouling Coating that Enables Affinity-Based Electrochemical 3212 Biosensing in Complex Biological Fluids. Nat. Nanotechnol. 2019, $321314,1143-1149$.

3214 (290) Sun, T. L.; Kurokawa, T.; Kuroda, S.; Ihsan, A. B.; Akasaki, T.; 3215 Sato, K.; Haque, M. A.; Nakajima, T.; Gong, J. P. Physical Hydrogels 3216 Composed of Polyampholytes Demonstrate High Toughness and 3217 Viscoelasticity. Nat. Mater. 2013, 12, 932-937.

3218 (291) Dong, Q.; Liu, J.; Song, L.; Shao, G. Novel Zwitterionic 3219 Inorganic-Organic Hybrids: Synthesis of Hybrid Adsorbents and 3220 Their Applications for $\mathrm{Cu}^{2+}$ Removal. J. Hazard. Mater. 2011, 186, 3221 1335-1342.

3222 (292) Liu, J.; Ma, Y.; Xu, T.; Shao, G. Preparation of Zwitterionic 3223 Hybrid Polymer and Its Application for the Removal of Heavy Metal 3224 Ions from Water. J. Hazard. Mater. 2010, 178, 1021-1029.

3225 (293) Yeom, J.; Guimaraes, P. P. G.; Ahn, H. M.; Jung, B.-K.; Hu, 3226 Q.; McHugh, K.; Mitchell, M. J.; Yun, C.-O.; Langer, R.; Jaklenec, A. 3227 Chiral Supraparticles for Controllable Nanomedicine. Adv. Mater. 3228 2020, 32, 1903878.
(294) Qi, H.; Zheng, W.; Zhou, X.; Zhang, C.; Zhang, L. A Mussel- 3229 Inspired Chimeric Protein as a Novel Facile Antifouling Coating. 3230 Chem. Commun. 2018, 54, 11328-11331.

3231

(295) Qi, H.; Zheng, W.; Zhang, C.; Zhou, X.; Zhang, L. Novel 3232 Mussel-Inspired Universal Surface Functionalization Strategy: Pro- 3233 tein-Based Coating with Residue-Specific Post-Translational Mod- 3234 ification in Vivo. ACS Appl. Mater. Interfaces 2019, 11, 12846-12853. 3235

(296) Yang, C.; He, G.; Zhang, A.; Wu, Q.; Zhou, L.; Hang, T.; Liu, 3236 D.; Xiao, S.; Chen, H.-J.; Liu, F.; et al. Injectable Slippery Lubricant- 3237 Coated Spiky Microparticles with Persistent and Exceptional 3238 Biofouling-Resistance. ACS Cent. Sci. 2019, 5, 250-258.

3239

(297) Badv, M.; Imani, S. M.; Weitz, J. I.; Didar, T. F. Lubricant- 3240 Infused Surfaces with Built-In Functional Biomolecules Exhibit 3241 Simultaneous Repellency and Tunable Cell Adhesion. ACS Nano 3242 2018, 12, 10890-10902.

3243

(298) Greene, G. W.; Ortiz, V.; Pozo-Gonzalo, C.; Moulton, S. E.; 3244 Wang, X.; Martin, L. L.; Michalczky, A.; Howlett, P. C. Lubricin 3245 Antiadhesive Coatings Exhibit Size-Selective Transport Properties 3246 that Inhibit Biofouling of Electrode Surfaces with Minimal Loss in 3247 Electrochemical Activity. Adv. Mater. Interfaces 2018, 5, $1701296 . \quad 3248$

(299) Silva, S. M.; Quigley, A. F.; Kapsa, R. M. I.; Greene, G. W.; 3249 Moulton, S. E. Lubricin on Platinum Electrodes: A Low-Impedance 3250 Protein-Resistant Surface Towards Biomedical Implantation. Chem- 3251 ElectroChem 2019, 6, 1939-1943.

3252

(300) Ruiz-Valdepeñas Montiel, V.; Sempionatto, J. R.; Esteban- 3253 Fernández de Ávila, B.; Whitworth, A.; Campuzano, S.; Pingarrón, J. 3254 M.; Wang, J. Delayed Sensor Activation Based on Transient Coatings: 3255 Biofouling Protection in Complex Biofluids. J. Am. Chem. Soc. 2018, 3256 140, 14050-14053.

3257

(301) Laschewsky, A.; Rosenhahn, A. Molecular Design of 3258 Zwitterionic Polymer Interfaces: Searching for the Difference. 3259 Langmuir 2019, 35, 1056-1071.

3260

(302) Quan, X.; Zhao, D.; Li, L.; Zhou, J. Understanding the 3261 Cellular Uptake of pH-Responsive Zwitterionic Gold Nanoparticles: 3262 A Computer Simulation Study. Langmuir 2017, 33, 14480-14489. 3263

(303) Liu, C.; Guo, J.; Tian, F.; Yang, N.; Yan, F.; Ding, Y.; Wei, J.; 3264 Hu, G.; Nie, G.; Sun, J. Field-Free Isolation of Exosomes from 3265 Extracellular Vesicles by Microfluidic Viscoelastic Flows. ACS Nano 3266 2017, 11, 6968-6976.

3267

(304) Lee, K.; Shao, H.; Weissleder, R.; Lee, H. Acoustic 3268 Purification of Extracellular Microvesicles. ACS Nano 2015, 9, 3269 2321-2327.

3270

(305) Wu, M.; Ouyang, Y.; Wang, Z.; Zhang, R.; Huang, P.-H.; 3271 Chen, C.; Li, H.; Li, P.; Quinn, D.; Dao, M.; et al. Isolation of 3272 Exosomes from Whole Blood by Integrating Acoustics and Micro- 3273 fluidics. Proc. Natl. Acad. Sci. U. S. A. 2017, 114, 10584-10589. 3274

(306) Bagheri, M.; Akbari, A.; Mirbagheri, S. A. Advanced Control 3275 of Membrane Fouling in Filtration Systems Using Artificial 3276 Intelligence and Machine Learning Techniques: A Critical Review. 3277 Process Saf. Environ. Prot. 2019, 123, 229.

3278

(307) Ko, J.; Bhagwat, N.; Yee, S. S.; Ortiz, N.; Sahmoud, A.; Black, 3279 T.; Aiello, N. M.; McKenzie, L.; O’Hara, M.; Redlinger, C.; et al. 3280 Combining Machine Learning and Nanofluidic Technology To 3281 Diagnose Pancreatic Cancer Using Exosomes. ACS Nano 2017, 11, 3282 $11182-11193$.

3283

(308) Ohayon, D.; Nikiforidis, G.; Savva, A.; Giugni, A.; Wustoni, S.; 3284 Palanisamy, T.; Chen, X.; Maria, I. P.; Di Fabrizio, E.; Costa, P. M. F. 3285 J.; et al. Biofuel Powered Glucose Detection in Bodily Fluids with an 3286 n-Type Conjugated Polymer. Nat. Mater. 2019, 1-8. 3287

(309) Lee, H.; Hong, Y. J.; Baik, S.; Hyeon, T.; Kim, D. H. Enzyme- 3288 Based Glucose Sensor: From Invasive to Wearable Device. Adv. 3289 Healthcare Mater. 2018, 7, 1701150. 3290

(310) Lee, H.; Song, C.; Hong, Y. S.; Kim, M. S.; Cho, H. R.; Kang, 3291 T.; Shin, K.; Choi, S. H.; Hyeon, T.; Kim, D.-H. Wearable/Disposable 3292 Sweat-Based Glucose Monitoring Device with Multistage Trans- 3293 dermal Drug Delivery Module. Sci. Adv. 2017, 3, No. e1601314. 3294

(311) Chen, Y.; Lu, S.; Zhang, S.; Li, Y.; Qu, Z.; Chen, Y.; Lu, B.; 3295 Wang, X.; Feng, X. Skin-like Biosensor System via Electrochemical 3296 
3297 Channels for Noninvasive Blood Glucose Monitoring. Sci. Adv. 2017, 3298 3, No. e1701629.

3299 (312) Rivera, K. R.; Pozdin, V. A.; Young, A. T.; Erb, P. D.; 3300 Wisniewski, N. A.; Magness, S. T.; Daniele, M. Integrated 3301 Phosphorescence-Based Photonic Biosensor (iPOB) for Monitoring 3302 Oxygen Levels in 3D Cell Culture Systems. Biosens. Bioelectron. 2019, 3303 123, 131-140.

3304 (313) Piya, R.; Zhu, Y.; Soeriyadi, A. H.; Silva, S. M.; Reece, P. J.; 3305 Gooding, J. J. Micropatterning of Porous Silicon Bragg Reflectors 3306 With Poly (Ethylene Glycol) to Fabricate Cell Microarrays: Towards 3307 Single Cell Sensing. Biosens. Bioelectron. 2019, 127, 229-235.

3308 (314) Parker, S. G.; Yang, Y.; Ciampi, S.; Gupta, B.; Kimpton, K.; 3309 Mansfeld, F. M.; Kavallaris, M.; Gaus, K.; Gooding, J. J. A 3310 Photoelectrochemical Platform for the Capture and Release of Rare 3311 Single Cells. Nat. Commun. 2018, 9, 2288. 